\title{
Group 9 and 10 Metal Complexes of an Ylide- Substituted Phosphine: Coordination versus Cyclometallation and Oxidative Addition
}

Thorsten Scherpf, Ilja Rodstein, Maurice Paaßen, and Viktoria H. Gessner*

Chair of Inorganic Chemistry II, Ruhr-Universität Bochum, Universitätsstraße 150, 44801

Bochum, Germany. 


\section{Table of Contents}

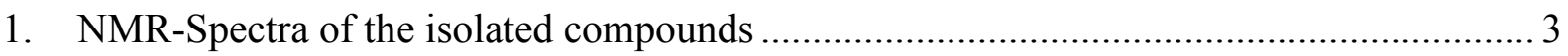

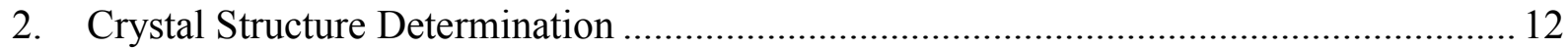

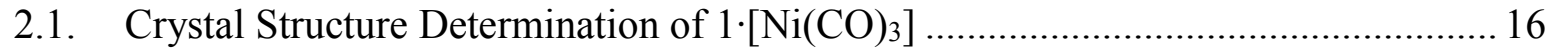

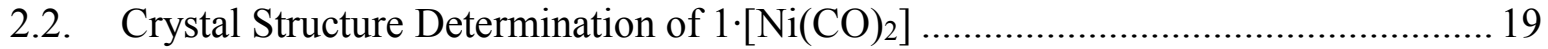

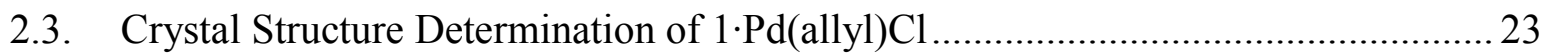

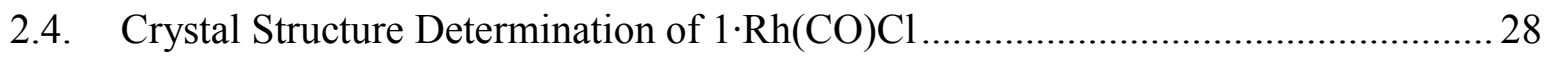

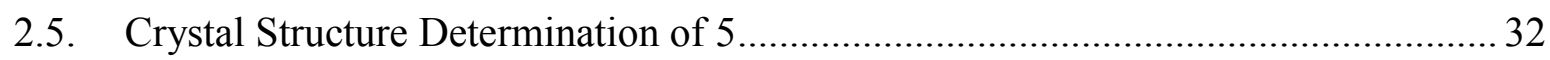

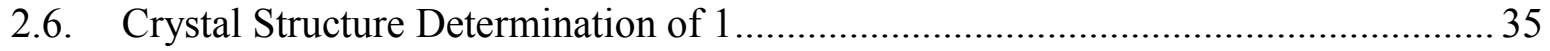

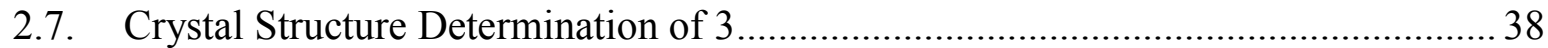

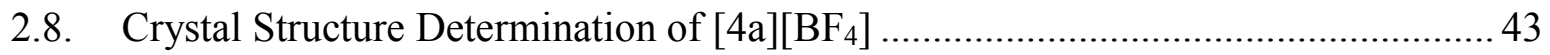

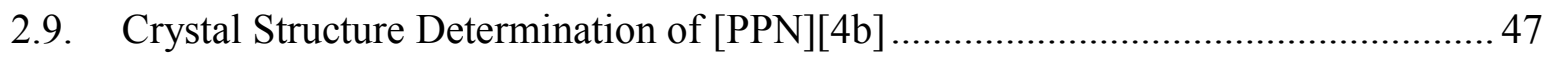

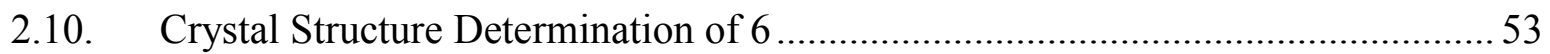




\section{NMR-Spectra of the isolated compounds}

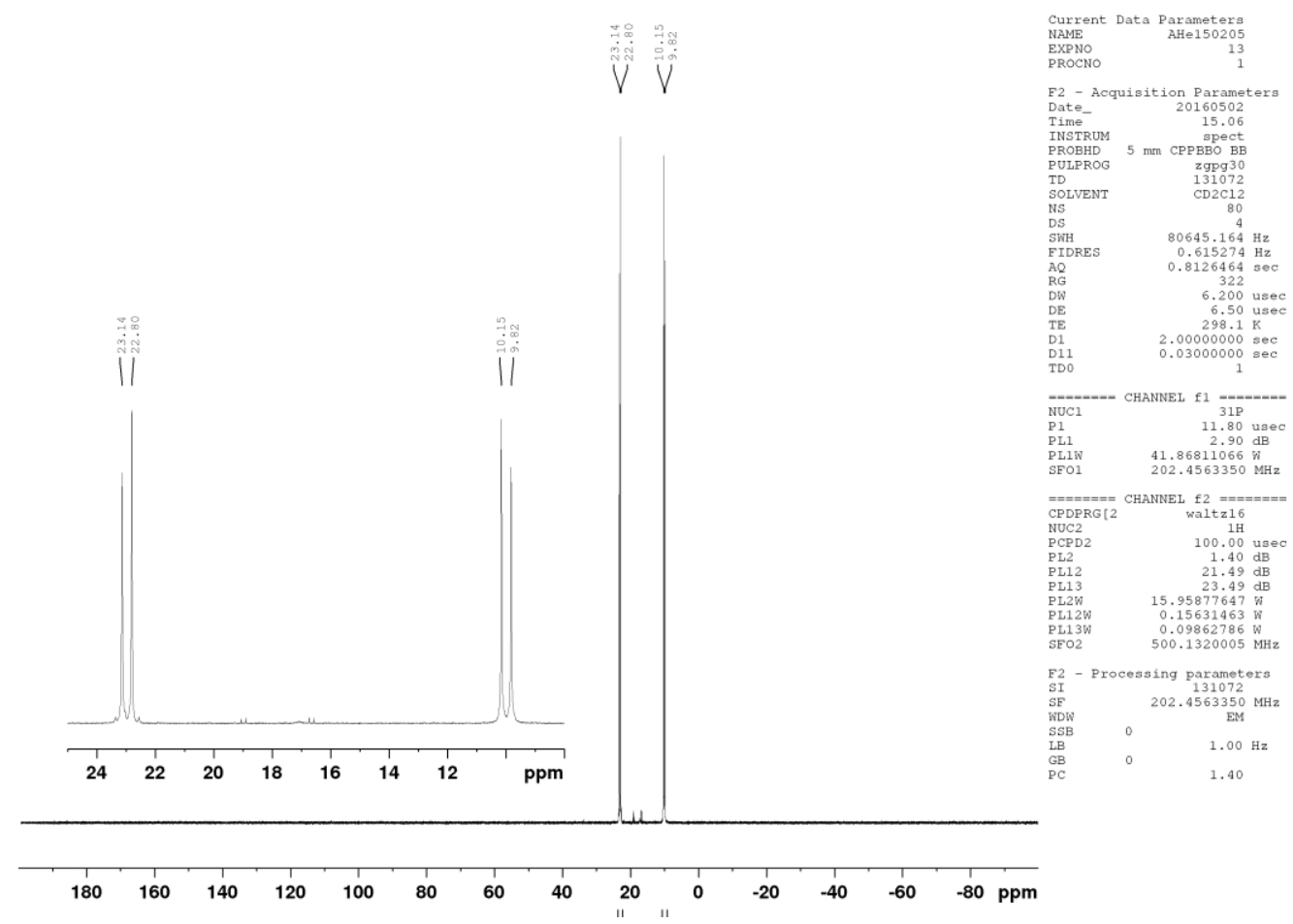

Figure S1: ${ }^{31} \mathrm{P}\left\{{ }^{1} \mathrm{H}\right\}$ NMR spectrum of $\mathbf{1} \cdot \mathrm{Pd}($ allyl $) \mathrm{Cl}$ in $\mathrm{CD}_{2} \mathrm{Cl}_{2}$.

guest Helbig

APROTON16_PRODI CD2C12 \{E: \Bruker \Topspin\} User 15

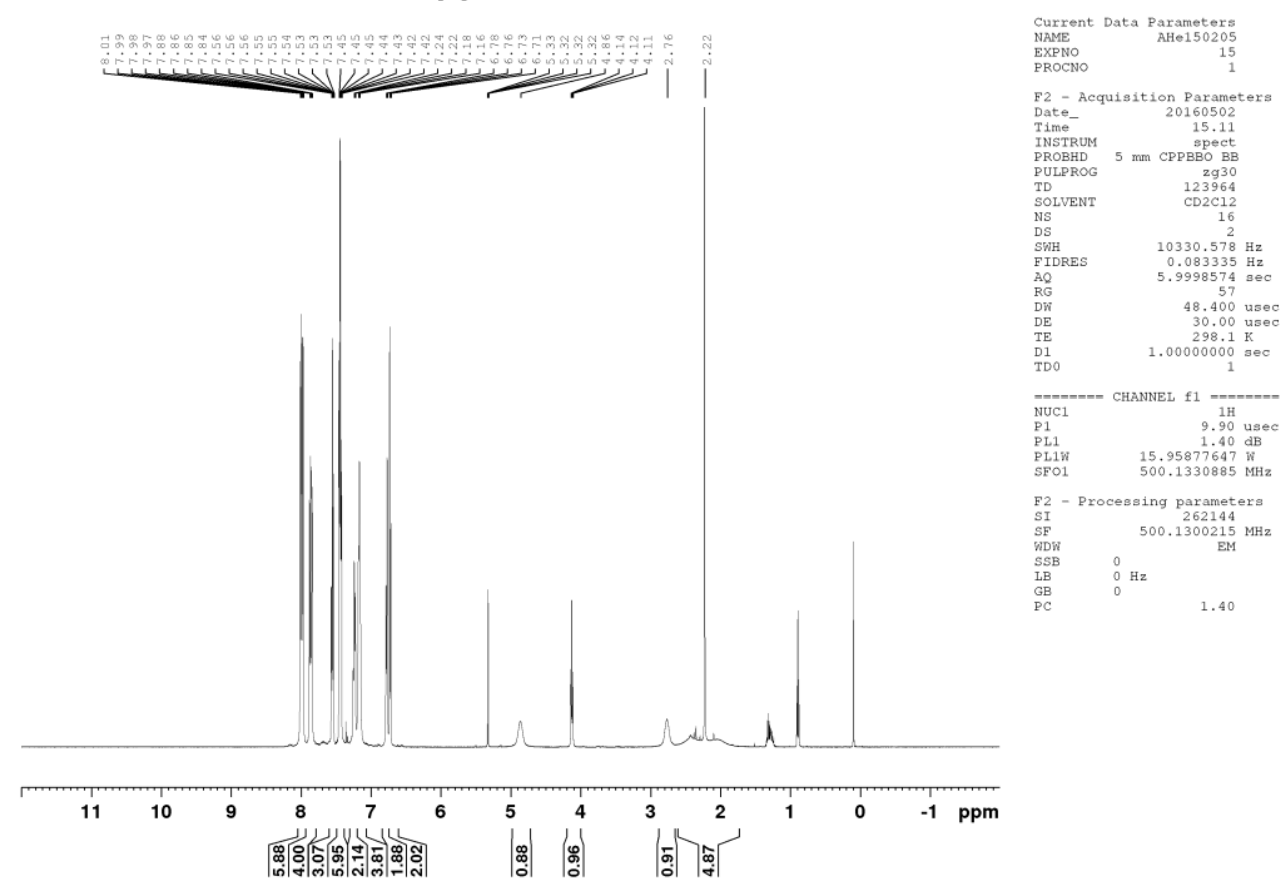

Figure S2: ${ }^{1} \mathrm{H}$ NMR spectrum of $1 \cdot \mathrm{Pd}($ allyl $) \mathrm{Cl}$ in $\mathrm{CD}_{2} \mathrm{Cl}_{2}$. 

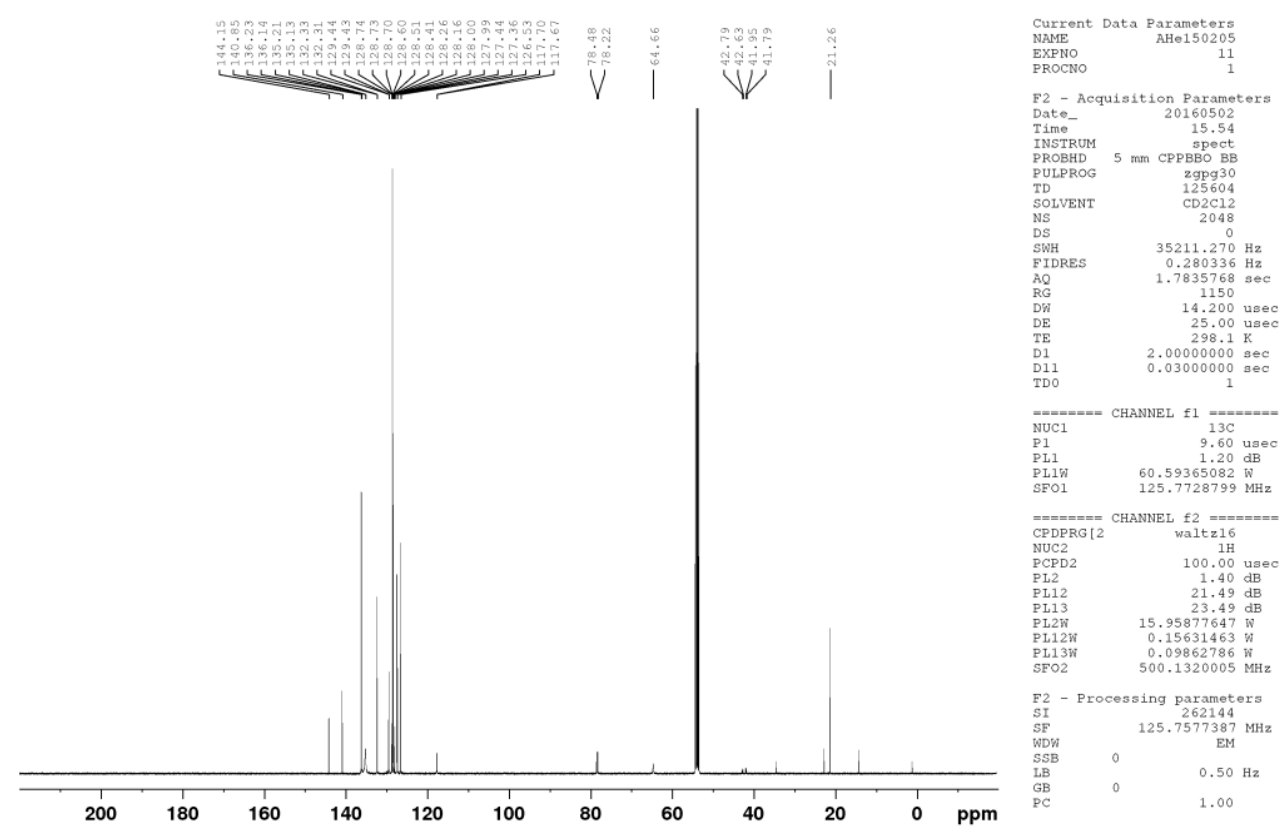

Figure S3: ${ }^{13} \mathrm{C}\left\{{ }^{1} \mathrm{H}\right\}$ NMR spectrum of $1 \cdot \mathrm{Pd}($ allyl $) \mathrm{Cl}$ in $\mathrm{CD}_{2} \mathrm{Cl}_{2}$.
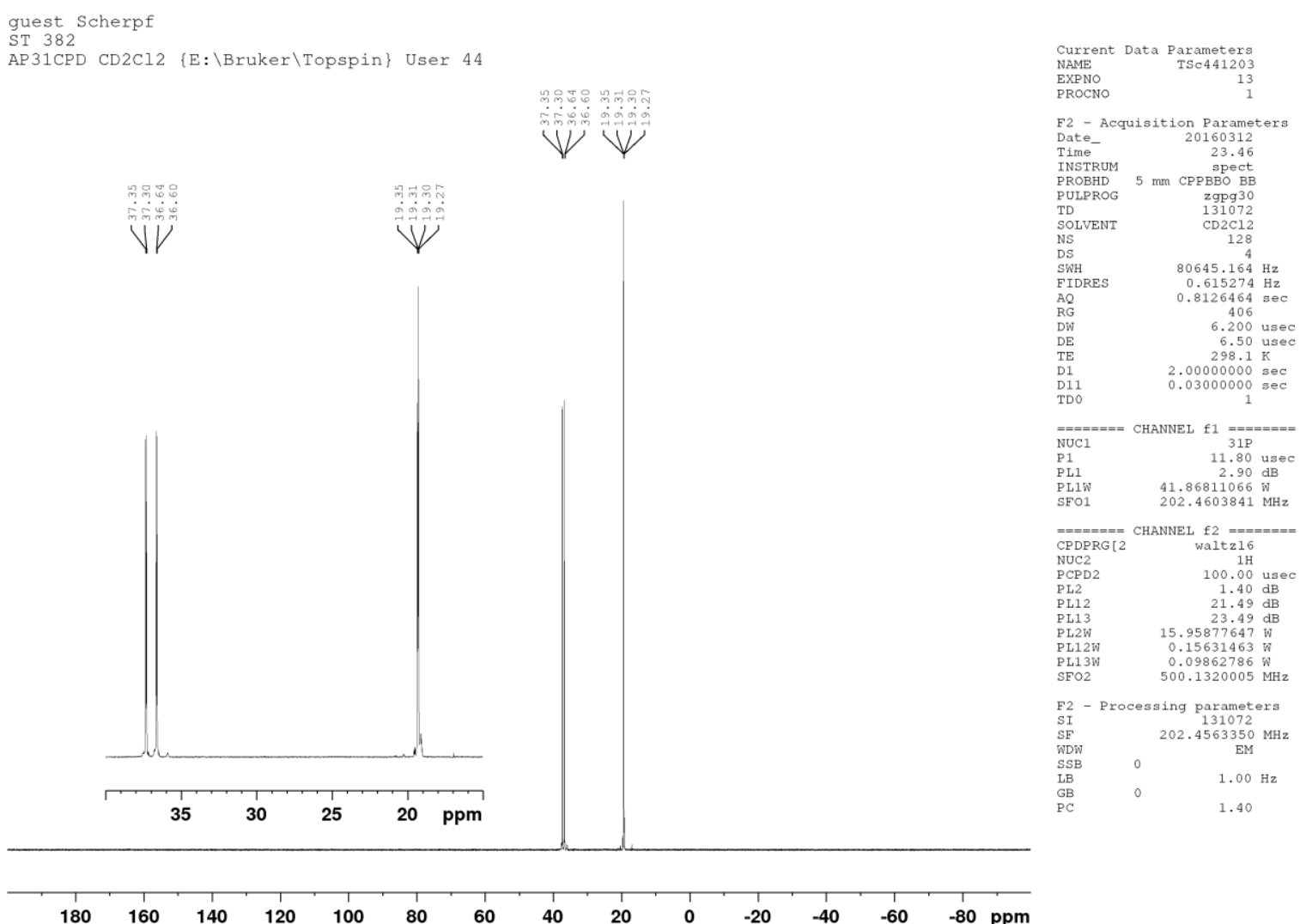

Figure S4: ${ }^{31} \mathrm{P}\left\{{ }^{1} \mathrm{H}\right\} \mathrm{NMR}$ spectrum of $\mathbf{1} \cdot \mathrm{Rh}(\mathrm{CO}) \mathrm{Cl}$ in $\mathrm{CD}_{2} \mathrm{Cl}_{2}$. 


\section{guest Scherpf}

ST 382

APROTON16_PRODI CD2Cl2 \{E: \Bruker $\backslash$ Topspin\} User 44
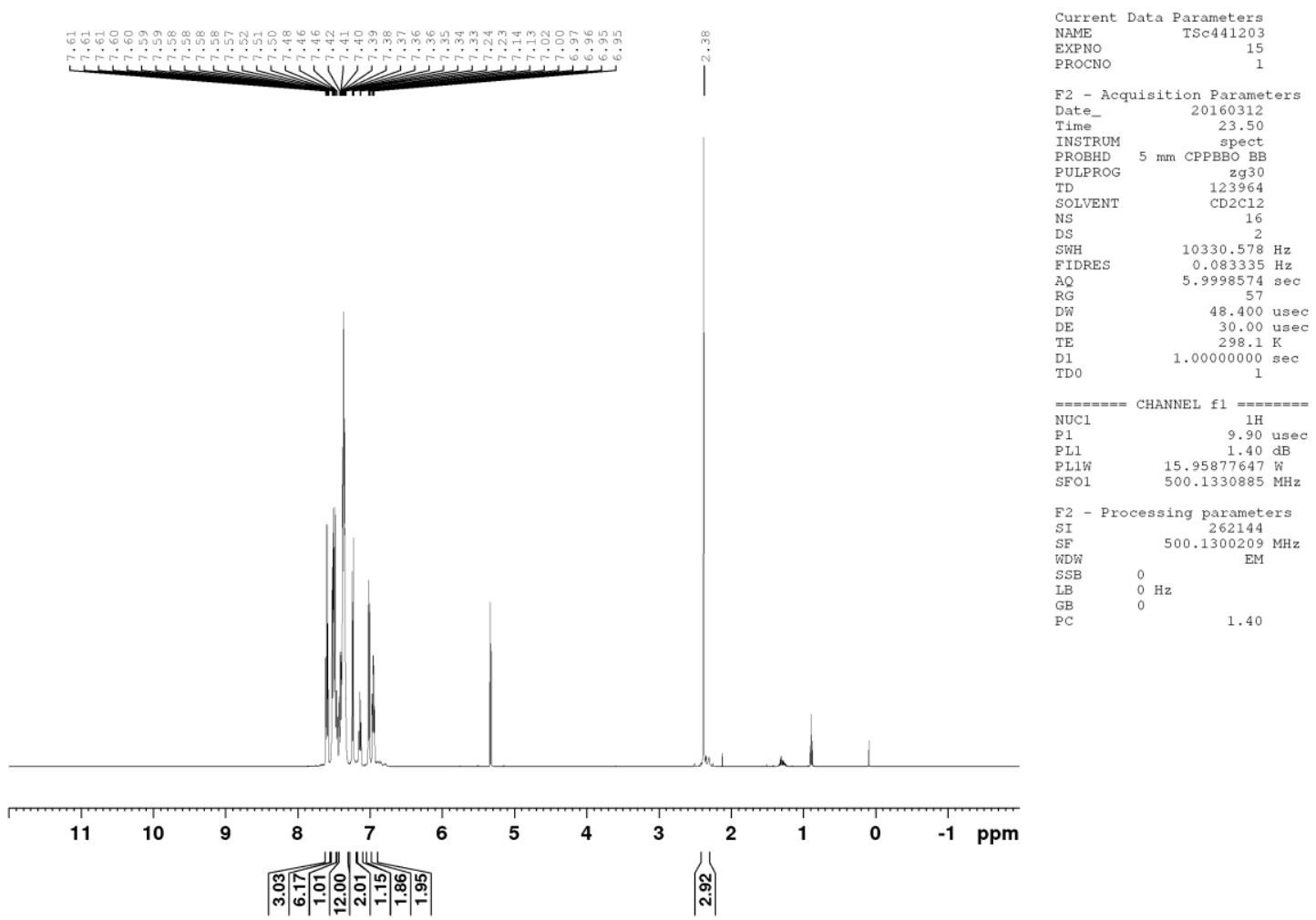

Figure S5: ${ }^{1} \mathrm{H}$ NMR spectrum of $\mathbf{1} \cdot \mathrm{Rh}(\mathrm{CO}) \mathrm{Cl}$ in $\mathrm{CD}_{2} \mathrm{Cl}_{2}$.

guest Scherpf
ST 382

AC13CPD_PRODI CD2C12 \{E: \Bruker $\backslash$ Topspin\} User 44
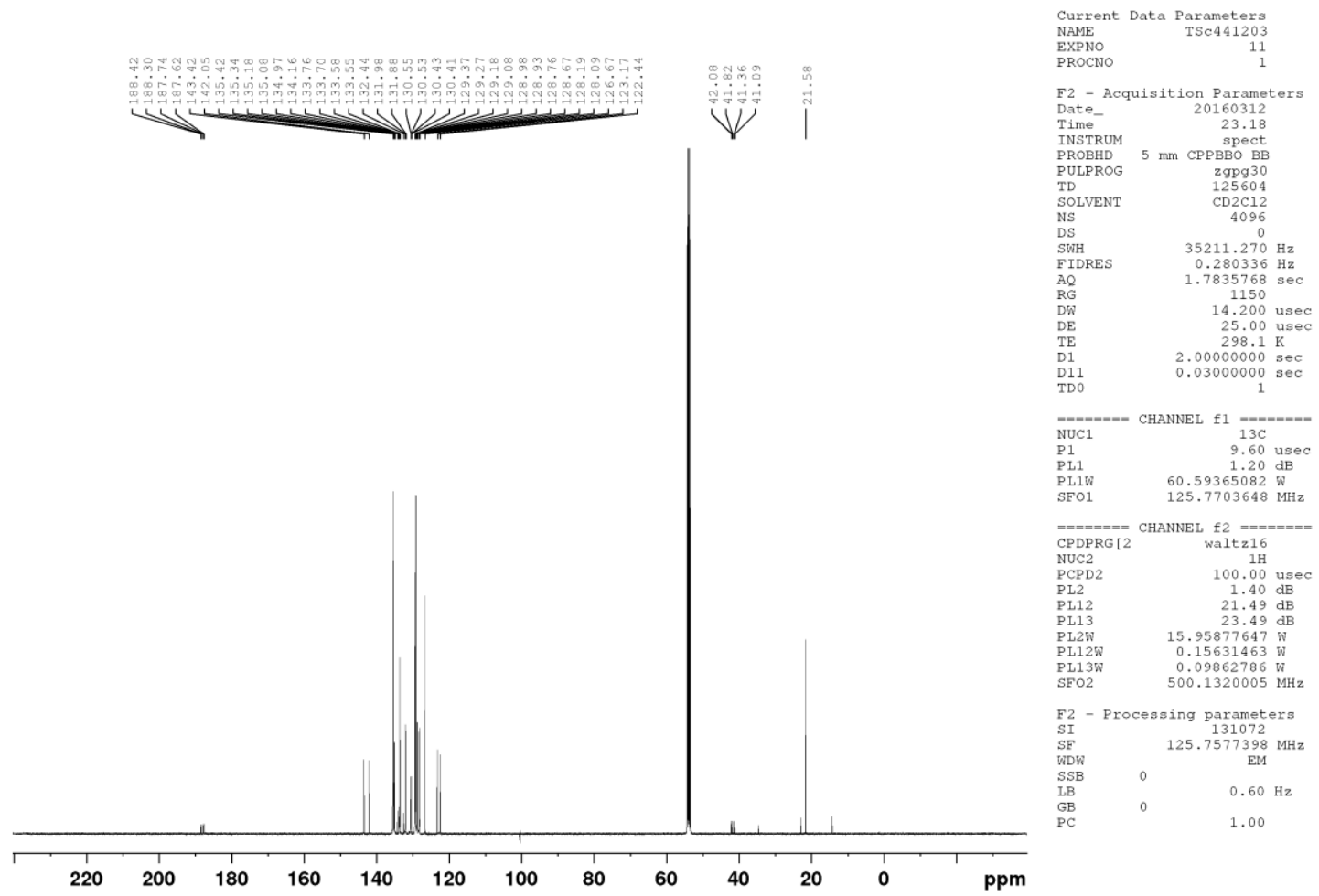

Figure S6: ${ }^{13} \mathrm{C}\left\{{ }^{1} \mathrm{H}\right\}$ NMR spectrum of $\mathbf{1} \cdot \mathrm{Rh}(\mathrm{CO}) \mathrm{Cl}$ in $\mathrm{CD}_{2} \mathrm{Cl}_{2}$. 


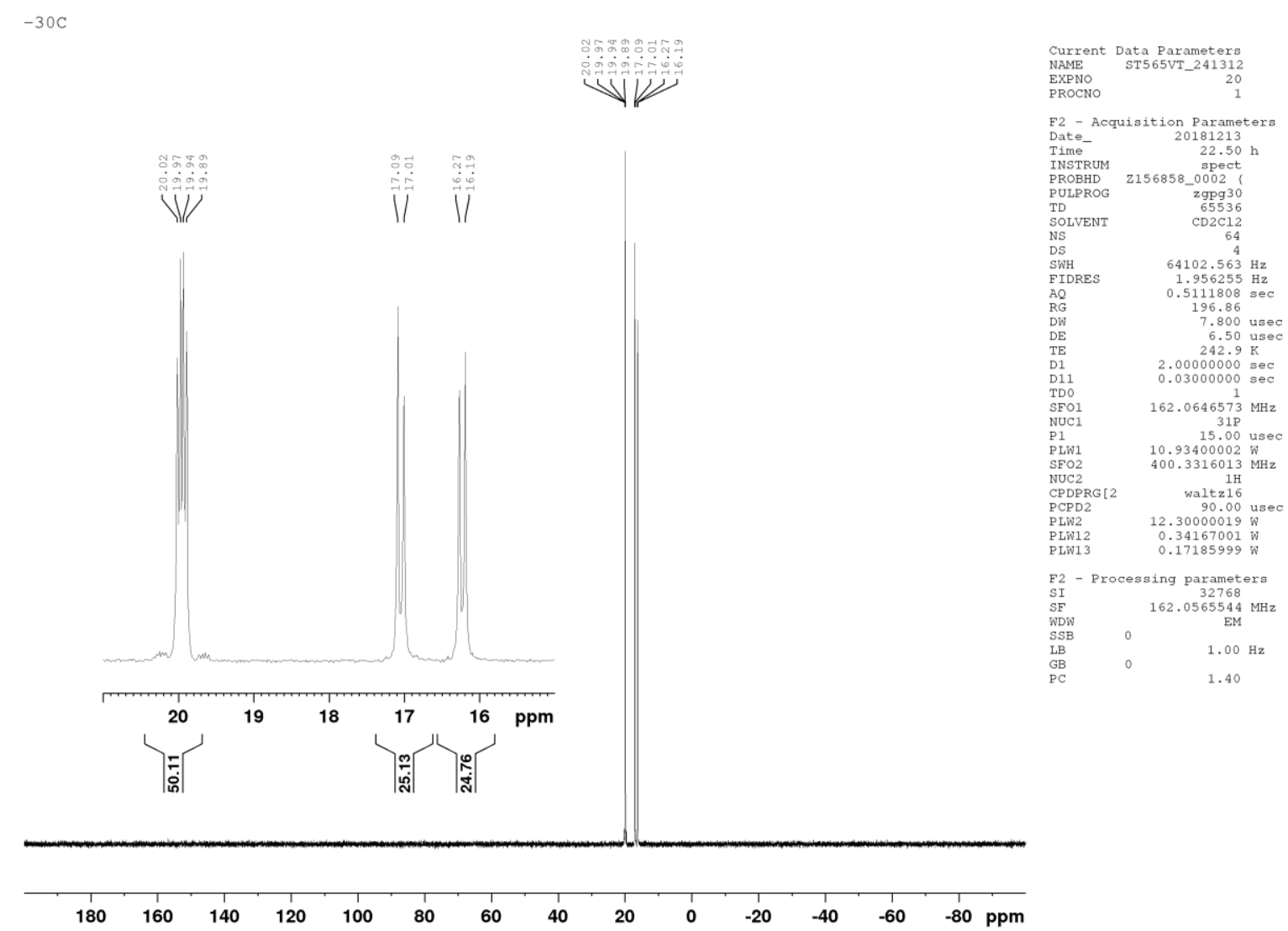

Figure S7: ${ }^{31} \mathrm{P}\left\{{ }^{1} \mathrm{H}\right\}$ NMR spectrum of $[4 \mathbf{a}]\left[\mathrm{BF}_{4}\right]$ in $\mathrm{CD}_{2} \mathrm{Cl}_{2}$ at $-30{ }^{\circ} \mathrm{C}$.

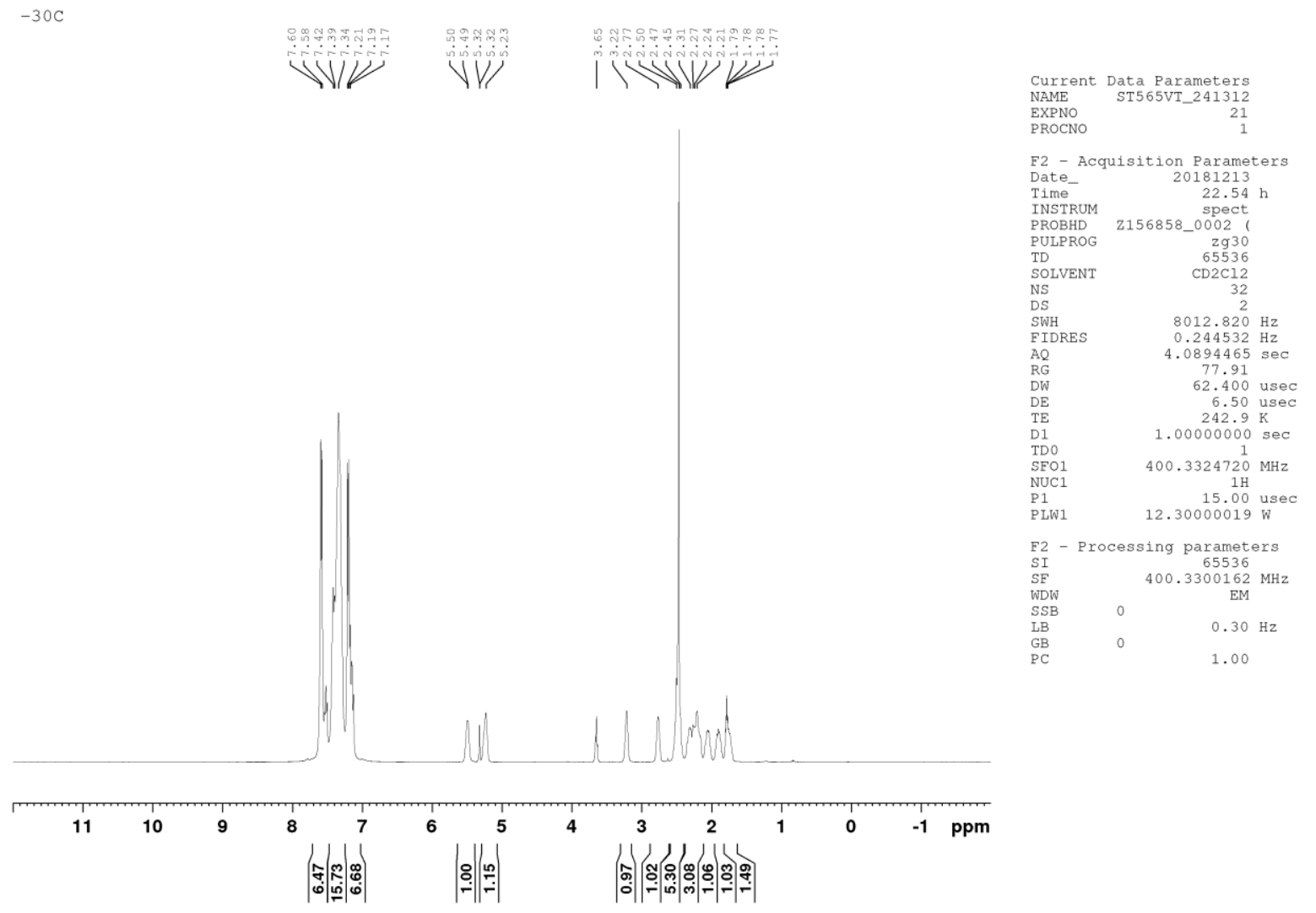

Figure S8: ${ }^{1} \mathrm{H}$ NMR spectrum of $[4 a]\left[\mathrm{BF}_{4}\right]$ in $\mathrm{CD}_{2} \mathrm{Cl}_{2}$ at $-30{ }^{\circ} \mathrm{C}$. 

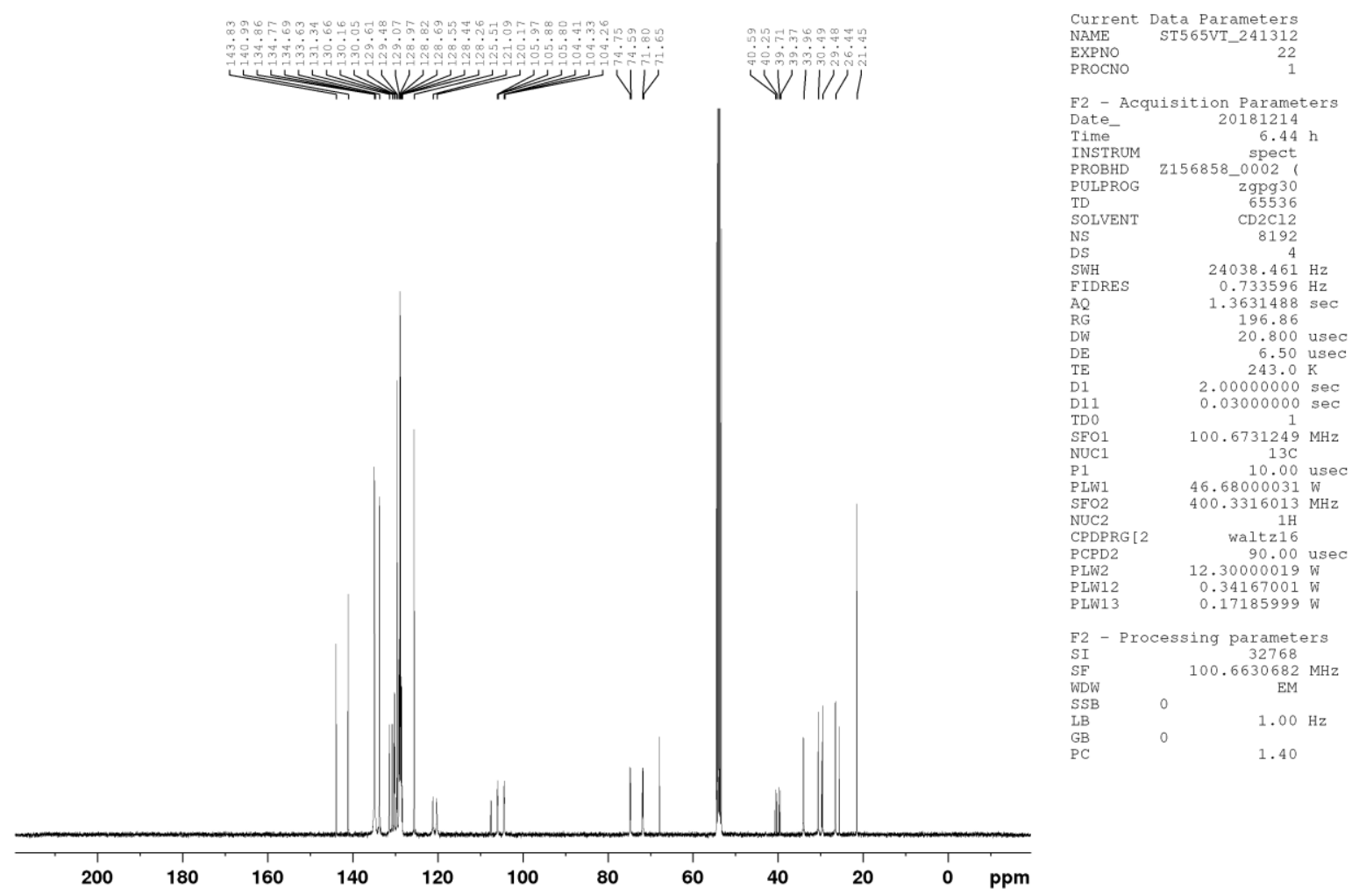

Figure S9: ${ }^{13} \mathrm{C}\left\{{ }^{1} \mathrm{H}\right\}$ NMR spectrum of $[4 \mathbf{a}]\left[\mathrm{BF}_{4}\right]$ in $\mathrm{CD}_{2} \mathrm{Cl}_{2}$ at $-30{ }^{\circ} \mathrm{C}$.

aus DCM/Et20

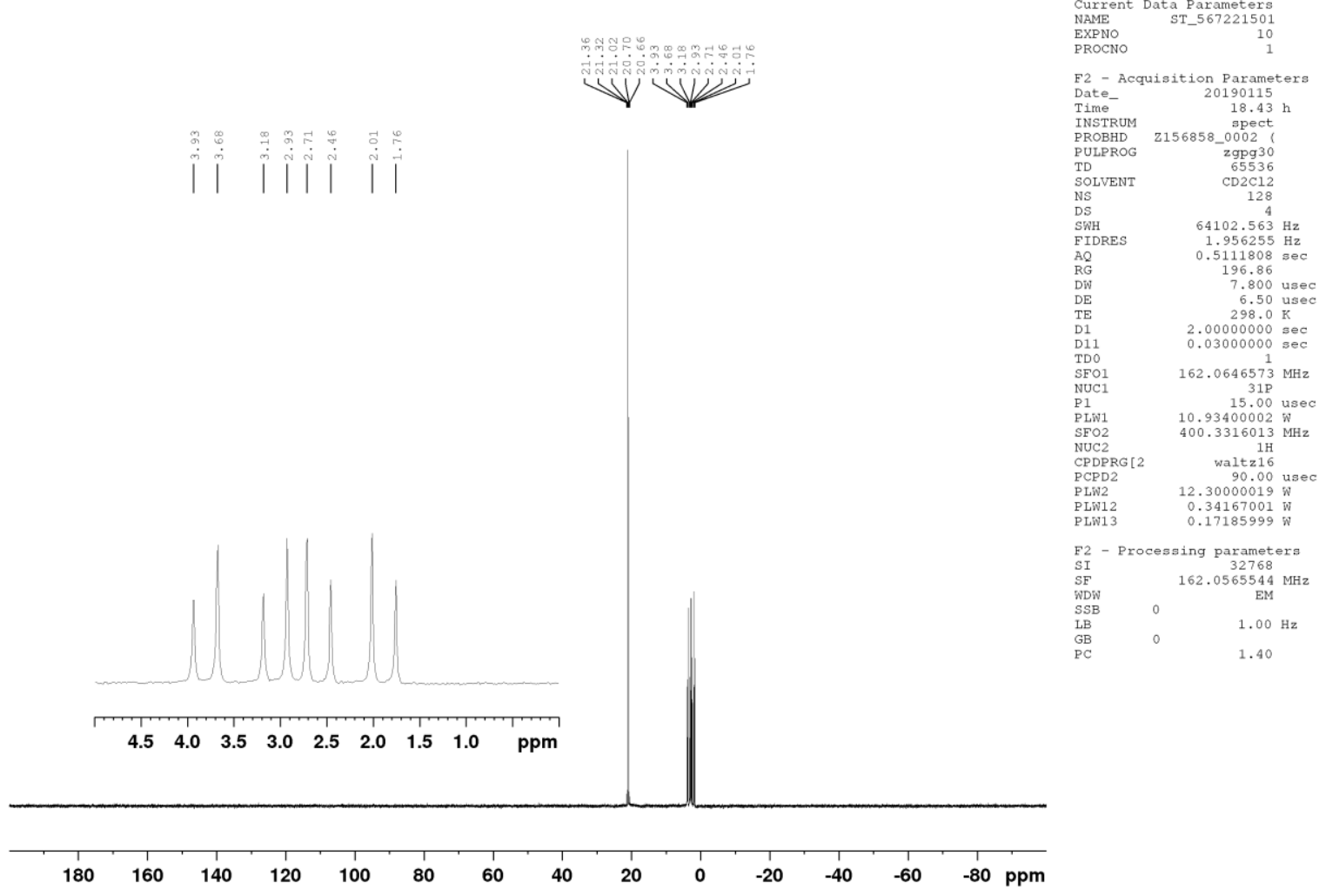

Figure S10: ${ }^{31} \mathrm{P}\left\{{ }^{1} \mathrm{H}\right\}$ NMR spectrum of $[\mathrm{PPN}][4 \mathbf{b}]$ in $\mathrm{CD}_{2} \mathrm{Cl}_{2}$. 


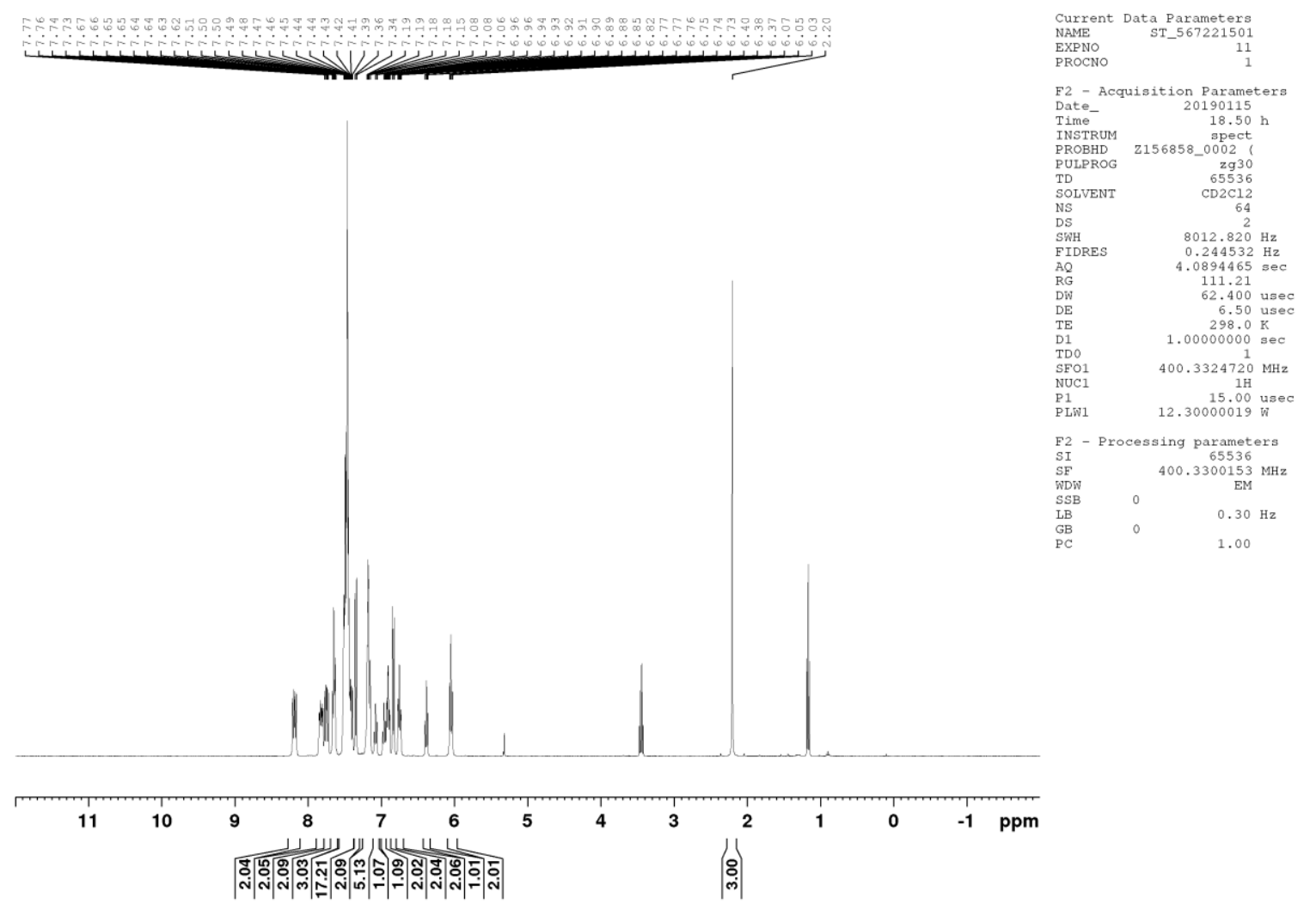

Figure S11: ${ }^{1} \mathrm{H}$ NMR spectrum of $[\mathrm{PPN}][\mathbf{4 b}]$ in $\mathrm{CD}_{2} \mathrm{Cl}_{2}$.

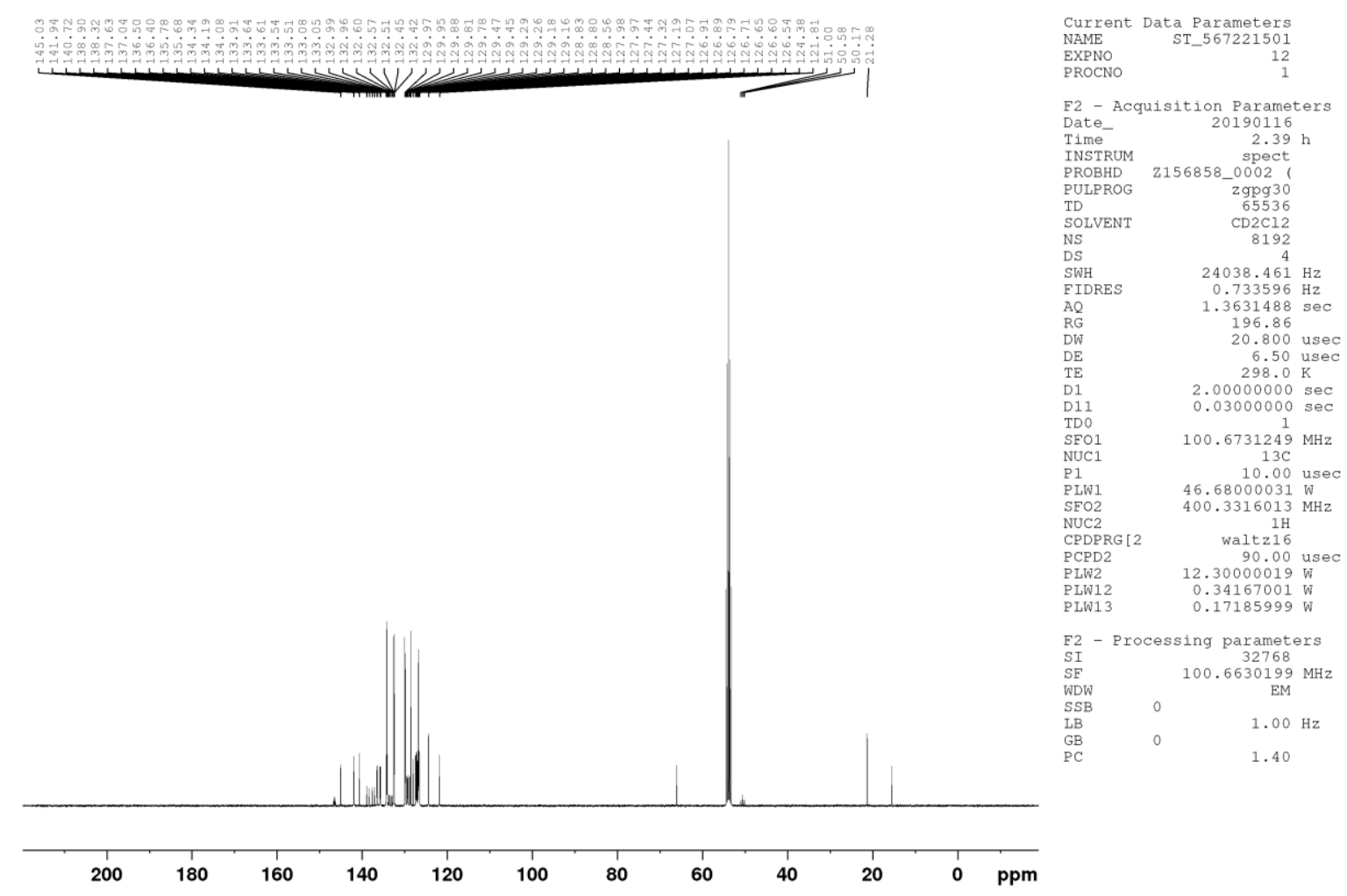

Figure S12: ${ }^{13} \mathrm{C}\left\{{ }^{1} \mathrm{H}\right\}$ NMR spectrum of $[\mathrm{PPN}][4 \mathbf{b}]$ in $\mathrm{CD}_{2} \mathrm{Cl}_{2}$. 


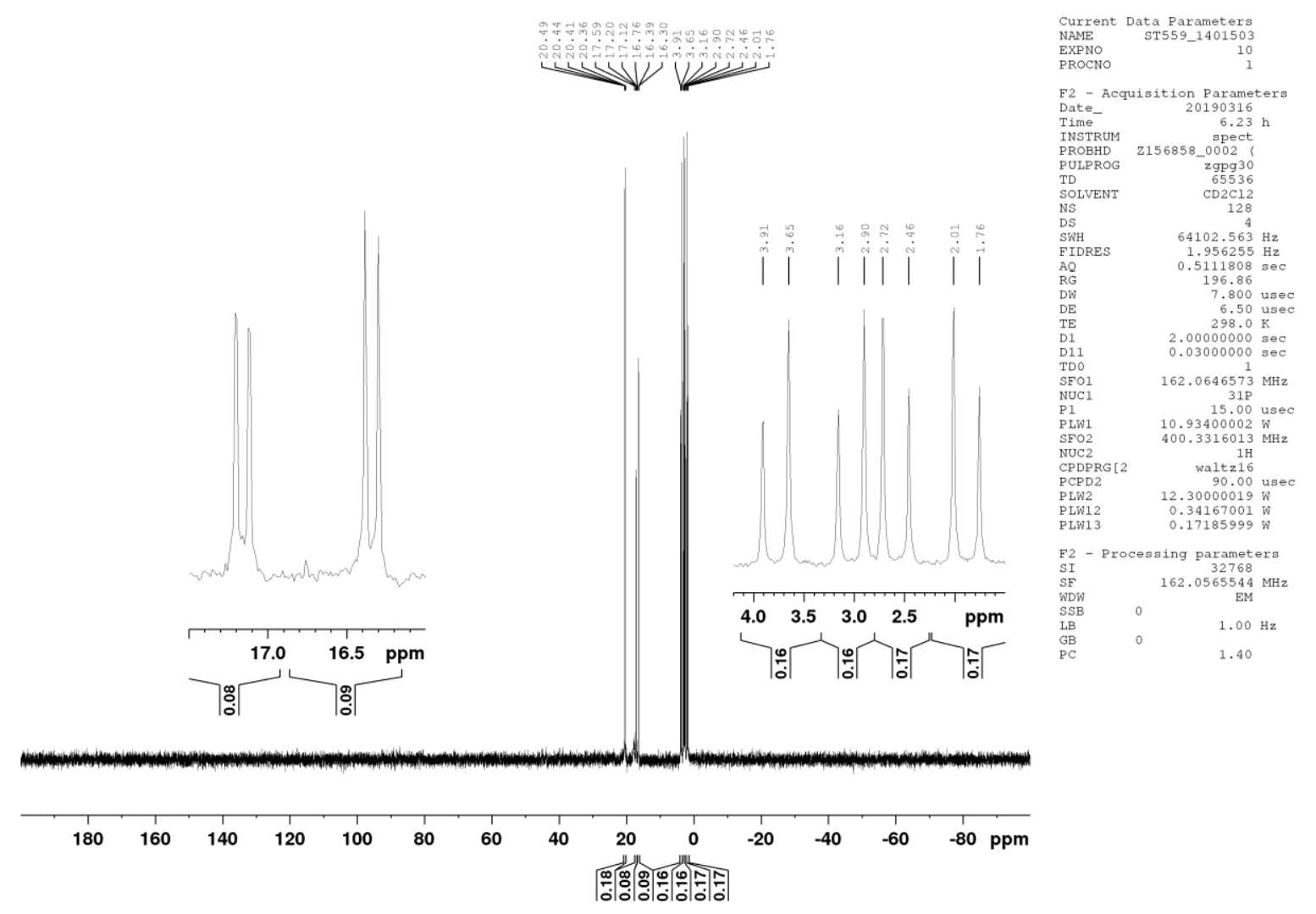

Figure S13: ${ }^{31} \mathrm{P}\left\{{ }^{1} \mathrm{H}\right\}$ NMR spectrum of $[\mathbf{4 a}][\mathbf{4 b}]$ in $\mathrm{CD}_{2} \mathrm{Cl}_{2}$.

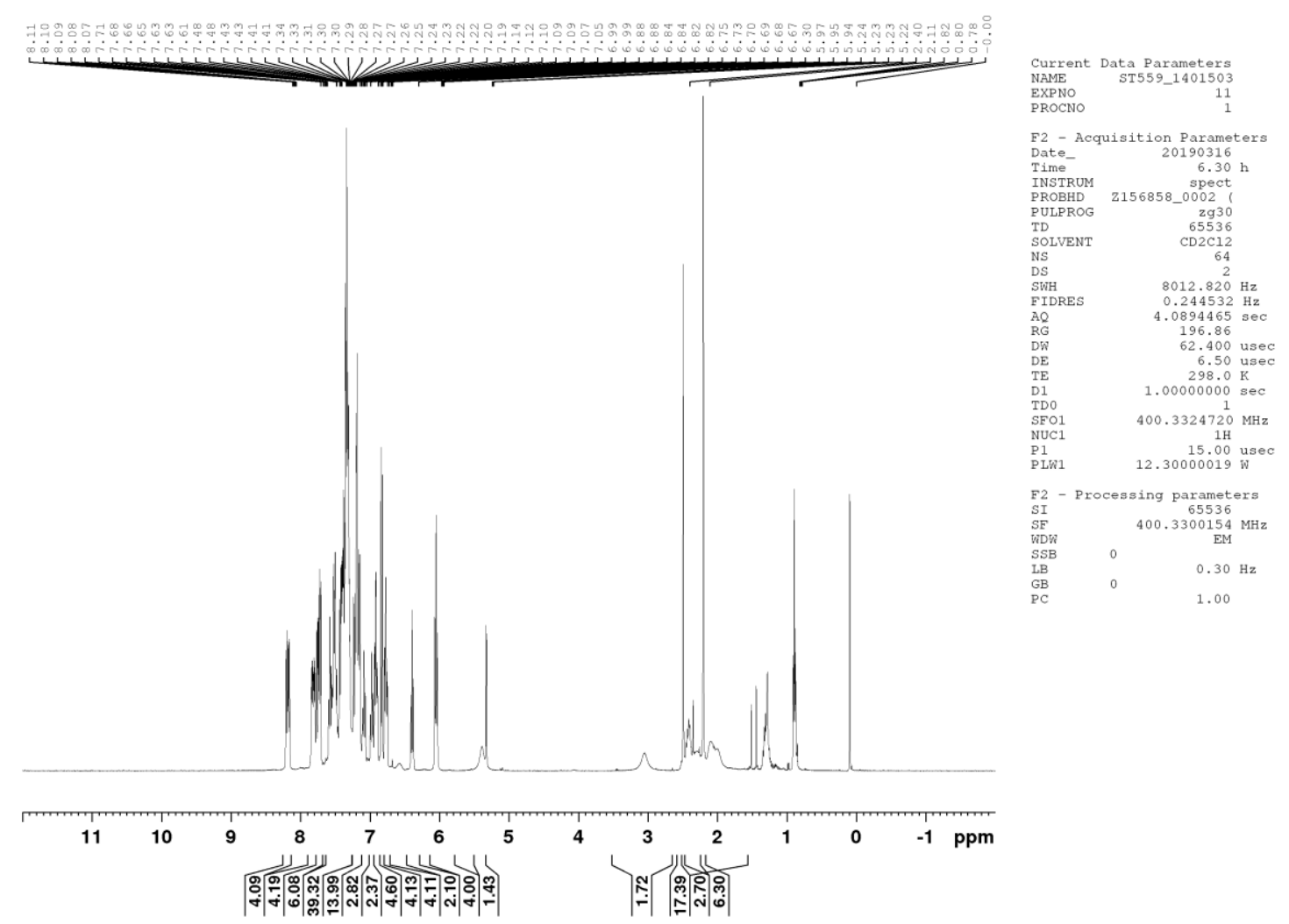

Figure S14: ${ }^{1} \mathrm{H}$ NMR spectrum of $[4 \mathbf{a}][4 \mathbf{b}]$ in $\mathrm{CD}_{2} \mathrm{Cl}_{2}$. 

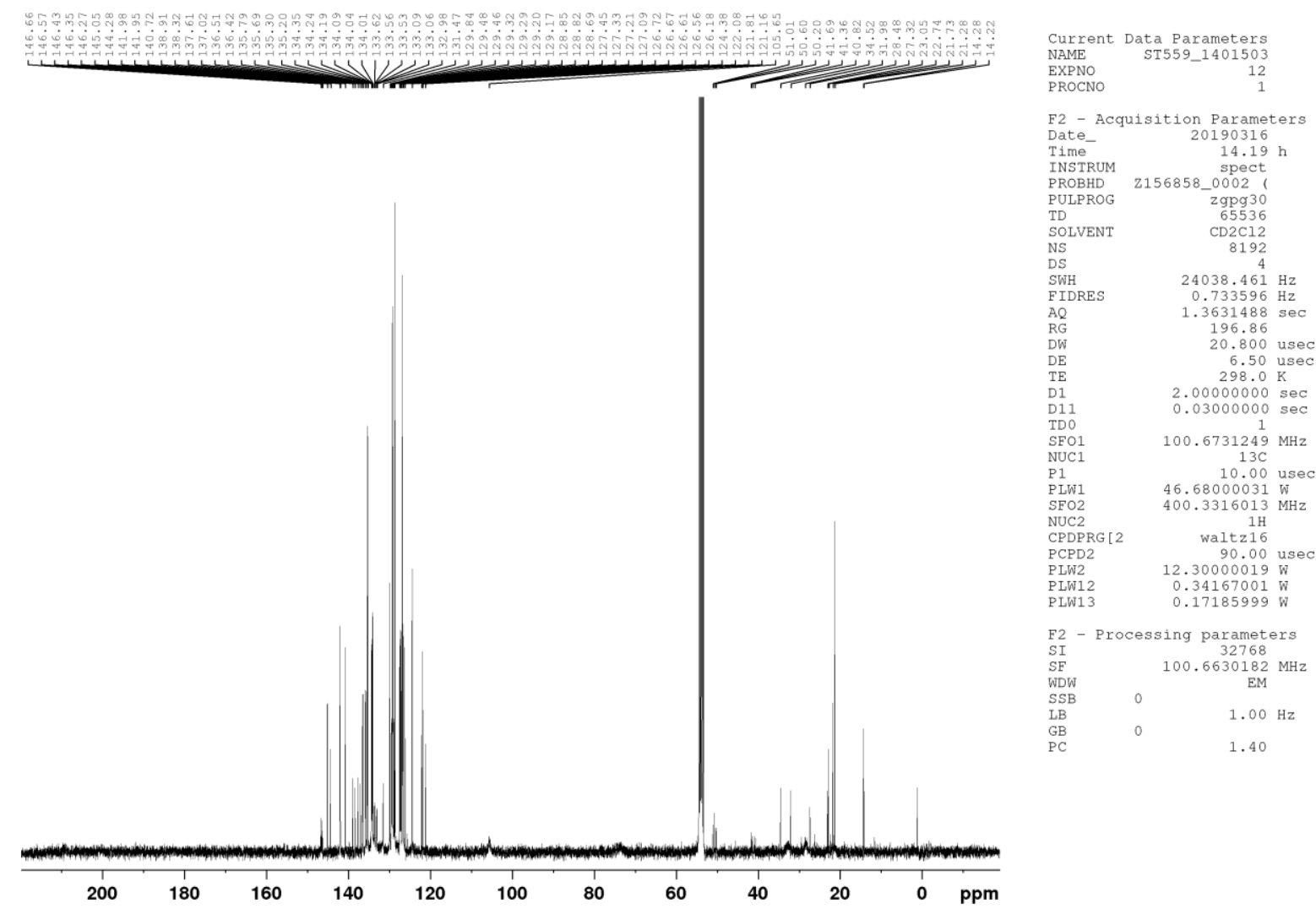

Figure S15: ${ }^{13} \mathrm{C}\left\{{ }^{1} \mathrm{H}\right\}$ NMR spectrum of $[\mathbf{4 a}][4 \mathbf{b}]$ in $\mathrm{CD}_{2} \mathrm{Cl}_{2}$.
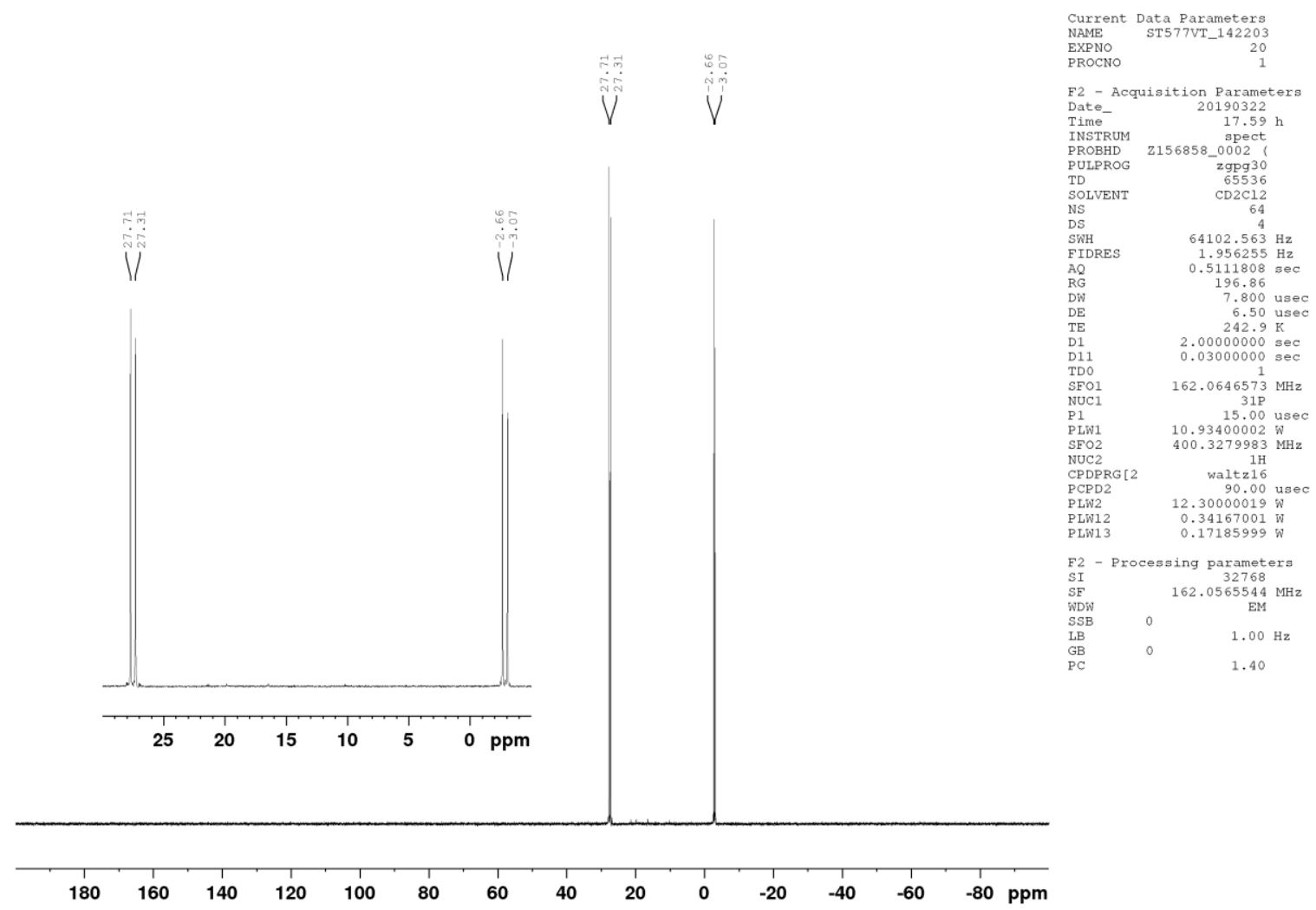

Figure S16: ${ }^{31} \mathrm{P}\left\{{ }^{1} \mathrm{H}\right\}$ NMR spectrum of 6 in $\mathrm{CD}_{2} \mathrm{Cl}_{2}$ at $-30^{\circ} \mathrm{C}$. 


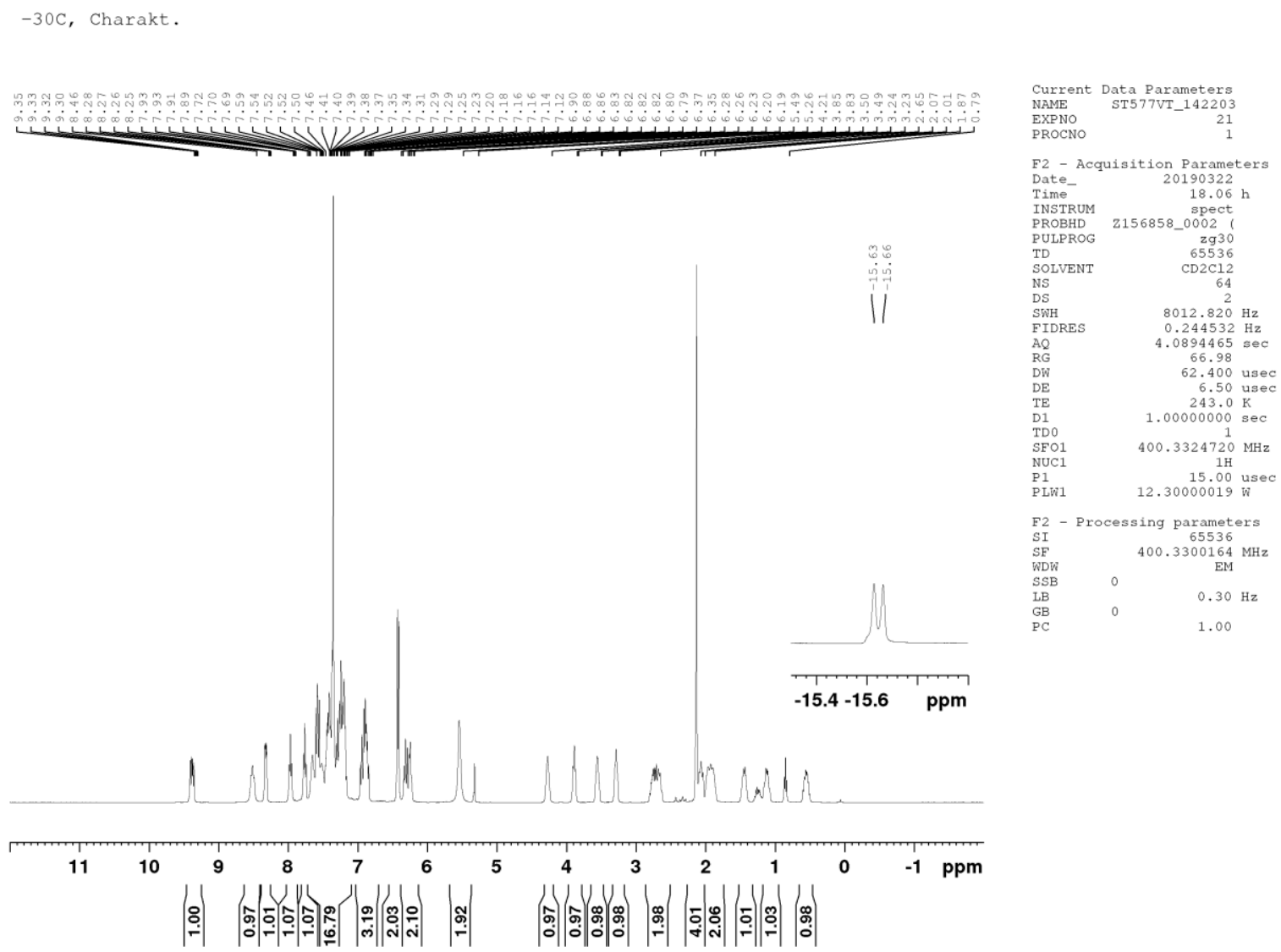

Figure S17: ${ }^{1} \mathrm{H}$ NMR spectrum of 6 in $\mathrm{CD}_{2} \mathrm{Cl}_{2}$ at $-30^{\circ} \mathrm{C}$.

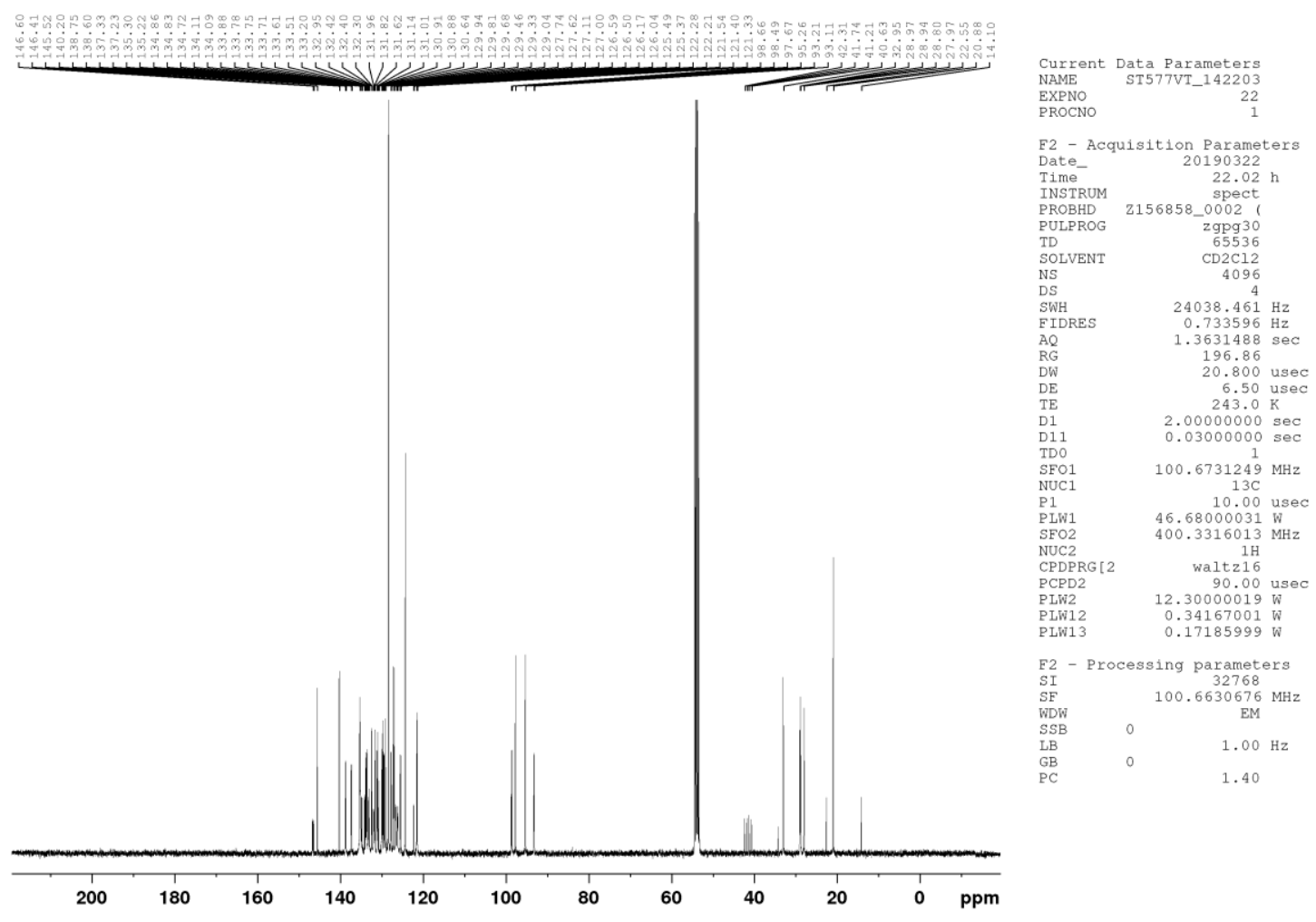

Figure S18: ${ }^{13} \mathrm{C}\left\{{ }^{1} \mathrm{H}\right\}$ NMR spectrum of $\mathbf{6}$ in $\mathrm{CD}_{2} \mathrm{Cl}_{2}$ at $-30^{\circ} \mathrm{C}$. 


\section{Crystal Structure Determination}

Table S1. Data collection and structure refinement details for compounds $\mathbf{1} \cdot \mathrm{Ni}(\mathrm{CO})_{3}, \mathbf{1} \cdot \mathrm{Ni}(\mathrm{CO})_{2}$ and $\mathbf{1} \cdot \mathrm{Pd}(\mathrm{allyl}) \mathrm{Cl}$.

\begin{tabular}{|c|c|c|c|}
\hline & $\mathbf{1} \cdot \mathrm{Ni}(\mathrm{CO})_{3}$ & $\mathbf{1} \cdot \mathrm{Ni}(\mathrm{CO})_{2}$ & 1.Pd(allyl)Cl \\
\hline CCDC No. & 1906075 & 1906074 & 1906076 \\
\hline Formula & $\mathrm{C}_{40} \mathrm{H}_{32} \mathrm{O}_{4} \mathrm{P}_{2} \mathrm{SNi}$ & $\mathrm{C}_{41} \mathrm{H}_{32} \mathrm{O}_{5} \mathrm{P}_{2} \mathrm{SNi}$ & $\mathrm{C}_{41.88} \mathrm{H}_{38.75} \mathrm{Cl}_{2.75} \mathrm{O}_{2} \mathrm{P}_{2} \mathrm{SPd}$ \\
\hline Formula weight $\left[\mathrm{g} \cdot \mathrm{mol}^{-1}\right]$ & 729.36 & 757.37 & 871.92 \\
\hline Temperature $[\mathrm{K}]$ & $100(2)$ & $100(2)$ & $100(2)$ \\
\hline Wave length $[\AA]]$ & 0.71073 & 0.71073 & 0.71073 \\
\hline Crystal system & Monoclinic & Monoclinic & Triclinic \\
\hline Space group & $P 2{ }_{1} / \mathrm{c}(14)$ & $P 2_{1} / \mathrm{n}(14)$ & $P-1(2)$ \\
\hline $\mathrm{a}[\AA]$ & $22.6514(13)$ & $9.6557(5)$ & $10.9099(7)$ \\
\hline $\mathrm{b}[\AA]$ & $13.0507(8)$ & $19.7365(11)$ & $19.0226(13)$ \\
\hline $\mathrm{c}[\AA]$ & $24.8321(15)$ & $18.6610(10)$ & 20.8891(14) \\
\hline$\alpha\left[^{\circ}\right]$ & 90 & 90 & $63.815(2)$ \\
\hline$\beta\left[^{\circ}\right]$ & $108.9456(17)$ & $91.3521(17)$ & $84.618(2)$ \\
\hline$\gamma\left[{ }^{\circ}\right]$ & 90 & 90 & $89.730(2)$ \\
\hline Volume $\left[\AA^{3}\right]$ & $6943.1(7)$ & $3555.2(3)$ & $3869.9(5)$ \\
\hline Z & 8 & 4 & 4 \\
\hline Calc. density $\left[\mathrm{Mg} \cdot \mathrm{m}^{-3}\right]$ & 1.395 & 1.415 & 1.497 \\
\hline$\mu\left(\mathrm{Mo}_{\mathrm{K} \alpha}\right)\left[\mathrm{mm}^{-1}\right]$ & 0.753 & 0.740 & 0.842 \\
\hline $\mathrm{F}(000)$ & 3024 & 1568 & 1779 \\
\hline Crystal dimensions [mm] & $0.42 \times 0.32 \times 0.14$ & $0.20 \times 0.20 \times 0.08$ & $0.12 \times 0.08 \times 0.03$ \\
\hline CCDC No. & 1.827 to 31.082 & 2.335 to 31.019 & 2.023 bis 24.999 \\
\hline Index ranges & $\begin{array}{l}-32 \leq \mathrm{h} \leq 31 \\
-17 \leq \mathrm{k} \leq 18 \\
-35 \leq 1 \leq 34\end{array}$ & $\begin{array}{l}-13 \leq \mathrm{h} \leq 13 \\
-27 \leq \mathrm{k} \leq 28 \\
-25 \leq 1 \leq 26\end{array}$ & $\begin{array}{l}-14 \leq \mathrm{h} \leq 14 \\
-25 \leq \mathrm{k} \leq 25 \\
-27 \leq 1 \leq 27\end{array}$ \\
\hline Reflections collected & 113465 & 57894 & 59453 \\
\hline Independent reflections & $20451\left[\mathrm{R}_{\text {int }}=0.0360\right]$ & $10498\left[\mathrm{R}_{\text {int }}=0.0397\right]$ & $19210\left[\mathrm{R}_{\mathrm{int}}=0.0835\right]$ \\
\hline Data/Restraints/Parameter & $20451 / 0 / 867$ & $10498 / 0$ / 452 & $19210 / 220 / 979$ \\
\hline Goodness-of-fit on $\mathrm{F}^{2}$ & 1.028 & 1.044 & 1.029 \\
\hline Final $R$ indices $[\mathrm{I}>2 \sigma(\mathrm{I})]$ & $\begin{array}{l}\mathrm{R} 1=0.0355 \\
\mathrm{wR} 2=0.0803\end{array}$ & $\begin{array}{l}\mathrm{R} 1=0.0379 \\
\mathrm{wR} 2=0.0846\end{array}$ & $\begin{array}{l}\mathrm{R} 1=0.0545 \\
\mathrm{wR} 2=0.1088\end{array}$ \\
\hline$R$ indices (all data) & $\begin{array}{l}\mathrm{R} 1=0.0522 \\
\mathrm{wR} 2=0.0867\end{array}$ & $\begin{array}{l}\mathrm{R} 1=0.0553 \\
\mathrm{wR} 2=0.0912\end{array}$ & $\begin{array}{l}\mathrm{R} 1=0.1036 \\
\mathrm{wR} 2=0.1254\end{array}$ \\
\hline $\begin{array}{l}\text { Largest diff. peak and } \\
\text { hole }\left[\mathrm{e} \cdot \AA^{-3}\right]\end{array}$ & 0.506 und -0.595 & 1.034 und -0.704 & 1.918 und -1.336 \\
\hline
\end{tabular}


Table S2. Data collection and structure refinement details for compounds $\mathbf{1} \cdot \mathrm{Rh}(\mathrm{CO}) \mathrm{Cl}$ and $\mathbf{5}$.

\begin{tabular}{|c|c|c|}
\hline & $\mathbf{1} \cdot \mathrm{Rh}(\mathrm{CO}) \mathrm{Cl}$ & 5 \\
\hline CCDC No. & 1906077 & 1906068 \\
\hline Formula & $\mathrm{C}_{46} \mathrm{H}_{39} \mathrm{Cl}_{4} \mathrm{O}_{3} \mathrm{P}_{2} \mathrm{SRh}$ & $\mathrm{C}_{79} \mathrm{H}_{73} \mathrm{Cl}_{11} \mathrm{~N}_{2} \mathrm{O}_{4} \mathrm{P}_{4} \mathrm{~S}_{2} \mathrm{Rh}_{2}$ \\
\hline Formula weight $\left[\mathrm{g} \cdot \mathrm{mol}^{-1}\right]$ & 729.36 & 1898.16 \\
\hline Temperature $[\mathrm{K}]$ & $100(2)$ & $100(2)$ \\
\hline Wave length $[\AA]$ & 0.71073 & 0.71073 \\
\hline Crystal system & Monoclinic & Triclinic \\
\hline Space group & $P 2_{1}(4)$ & $P-1(2)$ \\
\hline $\mathrm{a}[\AA]$ & $10.7770(8)$ & $10.1033(7)$ \\
\hline $\mathrm{b}[\AA]$ & $17.3118(13)$ & 13.1699(9) \\
\hline c $[\AA]$ & $12.6585(10)$ & $16.8526(11)$ \\
\hline$\alpha\left[^{\circ}\right]$ & 90 & $71.103(2)$ \\
\hline$\beta\left[^{\circ}\right]$ & $113.209(2)^{\circ}$. & $86.159(2)$ \\
\hline$\gamma\left[^{\circ}\right]$ & 90 & $68.211(2)$ \\
\hline Volume $\left[\AA^{3}\right]$ & $2170.6(3)$ & $1966.4(2))$ \\
\hline Z & 2 & 1 \\
\hline Calc. density $\left[\mathrm{Mg} \cdot \mathrm{m}^{-3}\right]$ & 1.497 & 1.603 \\
\hline$\mu\left(\mathrm{Mo}_{\mathrm{K} \alpha}\right)\left[\mathrm{mm}^{-1}\right]$ & 0.802 & 0.980 \\
\hline $\mathrm{F}(000)$ & 996 & 962 \\
\hline Crystal dimensions [mm] & $0.12 \times 0.10 \times 0.03$ & $0.27 \times 0.08 \times 0.06$ \\
\hline CCDC No. & 2.056 to $31.050^{\circ}$ & 1.759 to 31.336 \\
\hline \multirow{3}{*}{ Index ranges } & $-15 \leq h \leq 15$ & $-13 \leq h \leq 14$ \\
\hline & $-25 \leq \mathrm{k} \leq 24$ & $-19 \leq \mathrm{k} \leq 19$ \\
\hline & $-18 \leq 1 \leq 17$ & $-24 \leq 1 \leq 24$ \\
\hline Reflections collected & 43362 & 35619 \\
\hline Independent reflections & $12537\left[\mathrm{R}_{\mathrm{int}}=0.0344\right]$ & $11362\left[\mathrm{R}_{\mathrm{int}}=0.0362\right]$ \\
\hline Data/Restraints/Parameter & 12537 / $301 / 607$ & $11362 / 54 / 518$ \\
\hline Goodness-of-fit on $\mathrm{F}^{2}$ & 1.023 & 1.029 \\
\hline \multirow{2}{*}{ Final $\mathrm{R}$ indices $[\mathrm{I}>2 \sigma(\mathrm{I})]$} & $\mathrm{R} 1=0.0317$ & $\mathrm{R} 1=0.0352$ \\
\hline & $w R 2=0.0599$ & $w R 2=0.0727$ \\
\hline \multirow{2}{*}{$R$ indices (all data) } & $\mathrm{R} 1=0.0390$ & $\mathrm{R} 1=0.0521$ \\
\hline & $\mathrm{wR} 2=0.0623$ & $\mathrm{wR} 2=0.0784$ \\
\hline $\begin{array}{l}\text { Largest diff. peak and hole } \\
{\left[\mathrm{e} \cdot \AA^{-3}\right]}\end{array}$ & 0.790 und -0.398 & 0.709 und -0.602 \\
\hline
\end{tabular}


Table S3. Data collection and structure refinement details for compounds $\mathbf{1}, \mathbf{3}$ and $[\mathbf{4 a}]\left[\mathrm{BF}_{4}\right]$.

\begin{tabular}{|c|c|c|c|}
\hline & 1 & 3 & {$[\mathbf{4 a}]\left[\mathrm{BF}_{4}\right]$} \\
\hline CCDC No. & 1906072 & 1906069 & 1906070 \\
\hline Formula & $\mathrm{C}_{39.5} \mathrm{H}_{35} \mathrm{Cl}_{3} \mathrm{O}_{2} \mathrm{P}_{2} \mathrm{~S}$ & $\mathrm{C}_{72} \mathrm{H}_{70} \mathrm{O}_{6} \mathrm{P}_{4} \mathrm{~S}_{2} \mathrm{Pd}$ & $\mathrm{C}_{47} \mathrm{H}_{45} \mathrm{BO}_{2} \mathrm{~F}_{4} \mathrm{P}_{2} \mathrm{SCl}_{3} \mathrm{Rh}$ \\
\hline Formula weight $\left[\mathrm{g} \cdot \mathrm{mol}^{-1}\right]$ & 742.02 & 1325.68 & 1031.90 \\
\hline Temperature $[\mathrm{K}]$ & $100.00(10)$ & $100.00(10)$ & $100.00(10)$ \\
\hline Wave length $[\AA]$ & 1.54184 & 1.54184 & 1.54184 \\
\hline Crystal system & Triclinic & Triclinic & Monoclinic \\
\hline Space group & $P-1(2)$ & $P-1(2)$ & $C 2 / \mathrm{c}(15)$ \\
\hline $\mathrm{a}[\AA]$ & $9.96369(12)$ & $11.61275(8)$ & $36.01274(10)$ \\
\hline $\mathrm{b}[\AA]$ & $11.16620(16)$ & $12.92842(12)$ & $12.51873(4)$ \\
\hline $\mathrm{c}[\AA]$ & $17.0878(2)$ & $21.71534(16)$ & $20.15622(6)$ \\
\hline$\alpha\left[^{\circ}\right]$ & 77.4917(12) & $80.3998(7)$ & 90 \\
\hline$\beta\left[^{\circ}\right]$ & $87.5944(10)$ & $84.1038(6)$ & $94.3748(3)$ \\
\hline$\gamma\left[{ }^{\circ}\right]$ & $75.6120(11)$ & $74.1602(7)$ & 90 \\
\hline Volume $\left[\AA^{3}\right]$ & $1797.66(4)$ & $3086.87(4)$ & $3869.9(5)$ \\
\hline $\mathrm{Z}$ & 2 & 2 & 8 \\
\hline Calc. density $\left[\mathrm{Mg} \cdot \mathrm{m}^{-3}\right]$ & 1.371 & 1.426 & 1.513 \\
\hline$\mu\left(\mathrm{Cu}_{\mathrm{K} \alpha}\right)\left[\mathrm{mm}^{-1}\right]$ & 3.964 & 4.471 & 6.238 \\
\hline $\mathrm{F}(000)$ & 770 & 1376 & 4208 \\
\hline Crystal dimensions $[\mathrm{mm}]$ & $0.233 \times 0.140 \times 0.042$ & $0.135 \times 0.084 \times 0.033$ & $0.119 \times 0.117 \times 0.033$ \\
\hline CCDC No. & 2.649 to 74.499 & 2.067 to 72.126 & 2.461 to 74.500 \\
\hline \multirow{3}{*}{ Index ranges } & $-12 \leq \mathrm{h} \leq 12$ & $-14 \leq \mathrm{h} \leq 14$ & $-44 \leq \mathrm{h} \leq 44$ \\
\hline & $-11 \leq \mathrm{k} \leq 13$ & $-15 \leq \mathrm{k} \leq 15$ & $-15 \leq \mathrm{k} \leq 15$ \\
\hline & $-17 \leq 1 \leq 21$ & $-26 \leq 1 \leq 26$ & $-24 \leq 1 \leq 25$ \\
\hline Reflections collected & 28614 & 100183 & 77464 \\
\hline Independent reflections & $7320\left[\mathrm{R}_{\text {int }}=0.0186\right]$ & $12128\left[\mathrm{R}_{\mathrm{int}}=0.0249\right]$ & $9262\left[\mathrm{R}_{\text {int }}=0.0190\right]$ \\
\hline Data/Restraints/Parameter & $7320 / 98$ / 498 & $12128 / 152 / 859$ & 9262 / $290 / 645$ \\
\hline Goodness-of-fit on $\mathrm{F}^{2}$ & 1.048 & 1.128 & 1.036 \\
\hline Final R indices $[\mathrm{I}>2 \sigma(\mathrm{I})]$ & $\begin{array}{l}\mathrm{R} 1=0.0347 \\
\mathrm{wR} 2=0.0873\end{array}$ & $\begin{array}{l}\mathrm{R} 1=0.0222, \\
\mathrm{wR} 2=0.0660\end{array}$ & $\begin{array}{l}\mathrm{R} 1=0.0210 \\
\mathrm{wR} 2=0.0556\end{array}$ \\
\hline$R$ indices (all data) & $\begin{array}{l}\mathrm{R} 1=0.0353 \\
\mathrm{wR} 2=0.0877\end{array}$ & $\begin{array}{l}\mathrm{R} 1=0.0228 \\
\mathrm{wR} 2=0.0663\end{array}$ & $\begin{array}{l}\mathrm{R} 1=0.0211 \\
\mathrm{wR} 2=0.0556\end{array}$ \\
\hline $\begin{array}{l}\text { Largest diff. peak and } \\
\text { hole }\left[\mathrm{e} \cdot \AA^{-3}\right]\end{array}$ & 0.632 und -0.561 & 0.749 und -0.602 & 0.607 und -0.501 \\
\hline
\end{tabular}


Table S4. Data collection and structure refinement details for compounds [PPN][4b] and 6 .

\begin{tabular}{|c|c|c|}
\hline & {$[\mathrm{PPN}][4 \mathrm{~b}]$} & 6 \\
\hline CCDC No. & 1906071 & 1906073 \\
\hline Formula & $\begin{array}{l}\mathrm{C}_{130} \mathrm{H}_{112} \mathrm{NO}_{4} \mathrm{P}_{6} \mathrm{~S}_{2} \\
\mathrm{Cl}_{3} \mathrm{Rh}_{2}\end{array}$ & $\mathrm{C}_{46} \mathrm{H}_{44} \mathrm{O}_{2} \mathrm{P}_{2} \mathrm{SClIr}$ \\
\hline Formula weight $\left[\mathrm{g} \cdot \mathrm{mol}^{-1}\right]$ & 2314.31 & 950.46 \\
\hline Temperature $[\mathrm{K}]$ & $100.00(10)$ & $100.00(10)$ \\
\hline Wave length $[\AA]$ & 1.54184 & 1.54184 \\
\hline Crystal system & Monoclinic & Triclinic \\
\hline Space group & $P 2_{1} / \mathrm{c}(14)$ & $P-1(2)$ \\
\hline $\mathrm{a}[\AA]$ & $14.91330(8)$ & $12.58996(9)$ \\
\hline $\mathrm{b}[\AA]$ & $32.04084(16)$ & $13.93348(9)$ \\
\hline c $[\AA]$ & $22.75537(12)$ & $22.75785(14)$ \\
\hline$\alpha\left[^{\circ}\right]$ & 90 & $80.9153(5)$ \\
\hline$\beta\left[^{\circ}\right]$ & $97.1202(5)$ & $79.0576(6)$ \\
\hline$\gamma\left[{ }^{\circ}\right]$ & 90 & $83.5540(6)$ \\
\hline Volume $\left[\AA^{3}\right]$ & $10789.45(10)$ & $3856.51(5)$ \\
\hline $\mathrm{Z}$ & 4 & 4 \\
\hline Calc. density $\left[\mathrm{Mg} \cdot \mathrm{m}^{-3}\right]$ & 1.425 & 1.637 \\
\hline$\mu\left(\mathrm{Cu}_{\mathrm{K} \alpha}\right)\left[\mathrm{mm}^{-1}\right]$ & 4.808 & 8.935 \\
\hline $\mathrm{F}(000)$ & 4776 & 1904 \\
\hline Crystal dimensions $[\mathrm{mm}]$ & $0.362 \times 0.040 \times 0.023$ & $0.086 \times 0.067 \times 0.029$ \\
\hline CCDC No. & 2.394 to 77.166 & 3.224 to 77.518 \\
\hline Index ranges & $\begin{array}{c}-18 \leq \mathrm{h} \leq 18 \\
-39 \leq \mathrm{k} \leq 39 \\
-28 \leq 1 \leq 28\end{array}$ & $\begin{array}{l}-12 \leq \mathrm{h} \leq 15 \\
-17 \leq \mathrm{k} \leq 17 \\
-28 \leq 1 \leq 28\end{array}$ \\
\hline Reflections collected & 164815 & 57721 \\
\hline Independent reflections & $22467\left[\mathrm{R}_{\mathrm{int}}=0.0335\right]$ & $15973\left[\mathrm{R}_{\text {int }}=0.0329\right]$ \\
\hline Data/Restraints/Parameter & $22467 / 0 / 1335$ & 15973 / 28 / 987 \\
\hline Goodness-of-fit on $\mathrm{F}^{2}$ & 1.031 & 1.103 \\
\hline Final R indices $[\mathrm{I}>2 \sigma(\mathrm{I})]$ & $\begin{array}{l}\mathrm{R} 1=0.0337 \\
\mathrm{wR} 2=0.0872\end{array}$ & $\begin{array}{l}\mathrm{R} 1=0.0334, \\
\mathrm{wR} 2=0.0837\end{array}$ \\
\hline$R$ indices (all data) & $\begin{array}{l}\mathrm{R} 1=0.0372 \\
\mathrm{wR} 2=0.0905\end{array}$ & $\begin{array}{l}\mathrm{R} 1=0.0357 \\
\mathrm{wR} 2=0.0850\end{array}$ \\
\hline $\begin{array}{l}\text { Largest diff. peak and hole } \\
{\left[\mathrm{e} \cdot \AA^{-3}\right]}\end{array}$ & 1.205 und -1.025 & 2.002 und -2.023 \\
\hline
\end{tabular}




\subsection{Crystal Structure Determination of $1 \cdot \mathrm{Ni}(\mathrm{CO})_{3}$}

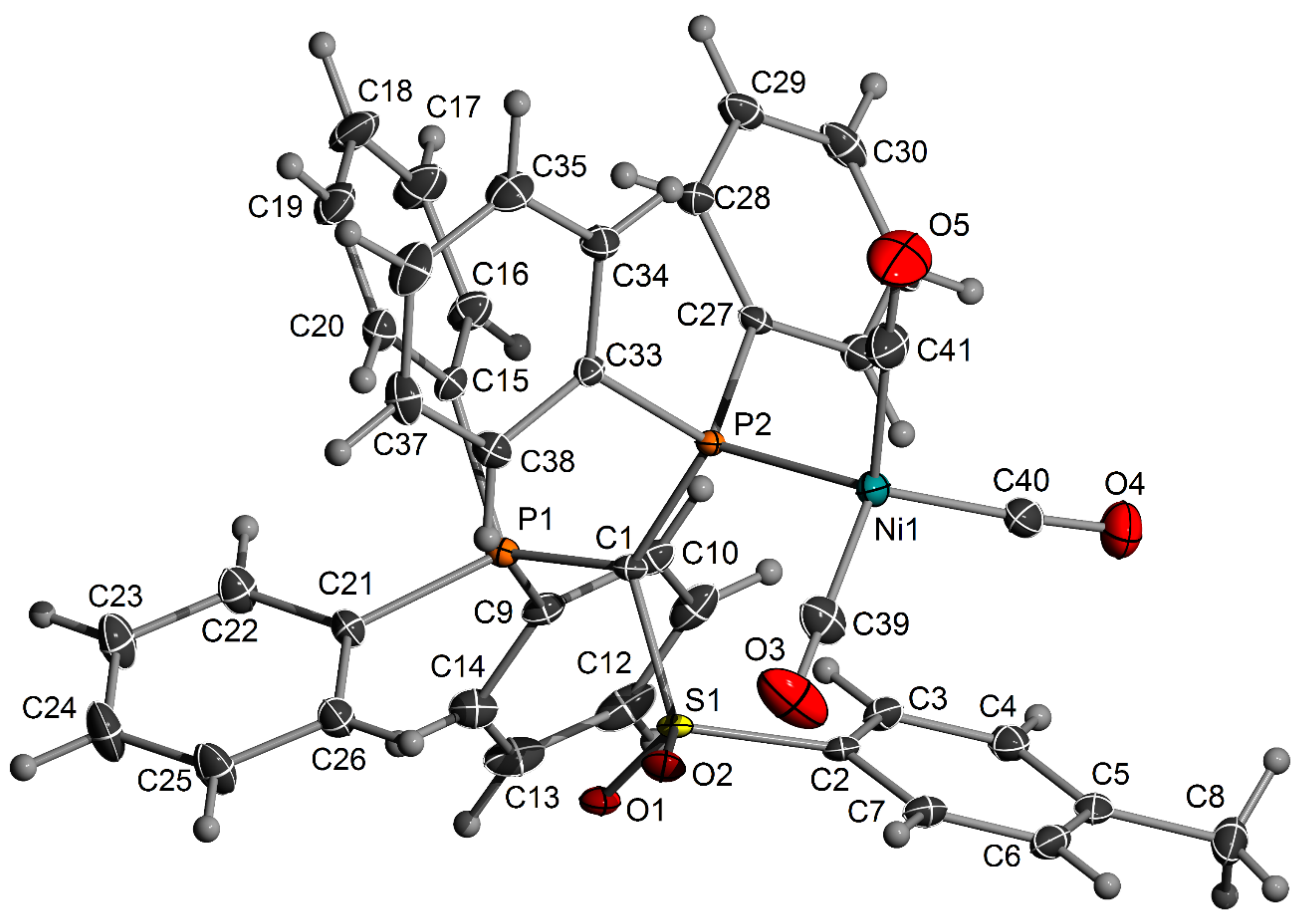

Figure S19. ORTEP Plot of $\mathbf{1} \cdot \mathrm{Ni}(\mathrm{CO})_{3}$. Ellipsoids are drawn at the $50 \%$ probability level.

Table S5. Atomic coordinates ( $\left.\mathrm{x} 10^{4}\right)$ and equivalent isotropic displacement parameters $\left(\AA^{2} \times 10^{3}\right)$ for $\mathbf{1} \cdot \mathrm{Ni}(\mathrm{CO})_{3}$. U(eq) is defined as one third of the trace of the orthogonalized $\mathrm{Uij}^{\mathrm{ij}}$ tensor.

\begin{tabular}{ccccc}
\hline & $\mathrm{x}$ & $\mathrm{y}$ & $\mathrm{z}$ & $\mathrm{U}(\mathrm{eq})$ \\
\hline $\mathrm{Ni}(1)$ & $8533(1)$ & $906(1)$ & $4653(1)$ & $14(1)$ \\
$\mathrm{S}(1)$ & $7564(1)$ & $2276(1)$ & $6008(1)$ & $10(1)$ \\
$\mathrm{P}(1)$ & $6398(1)$ & $3304(1)$ & $5084(1)$ & $11(1)$ \\
$\mathrm{P}(2)$ & $7544(1)$ & $1924(1)$ & $4434(1)$ & $10(1)$ \\
$\mathrm{O}(1)$ & $7012(1)$ & $2817(1)$ & $6451(1)$ & $14(1)$ \\
$\mathrm{O}(2)$ & $9026(1)$ & $2129(1)$ & $6108(1)$ & $15(1)$ \\
$\mathrm{O}(3)$ & $10992(2)$ & $1073(1)$ & $5600(1)$ & $40(1)$ \\
$\mathrm{O}(4)$ & $6503(2)$ & $-99(1)$ & $5118(1)$ & $35(1)$ \\
$\mathrm{O}(5)$ & $9624(2)$ & $457(1)$ & $3280(1)$ & $46(1)$ \\
$\mathrm{C}(1)$ & $7133(2)$ & $2498(1)$ & $5144(1)$ & $10(1)$ \\
$\mathrm{C}(2)$ & $6672(2)$ & $1524(1)$ & $6252(1)$ & $12(1)$ \\
$\mathrm{C}(3)$ & $5232(2)$ & $1505(1)$ & $6188(1)$ & $16(1)$ \\
$\mathrm{C}(4)$ & $4520(2)$ & $950(1)$ & $6444(1)$ & $17(1)$ \\
$\mathrm{C}(5)$ & $5218(2)$ & $413(1)$ & $6778(1)$ & $17(1)$ \\
$\mathrm{C}(6)$ & $6657(2)$ & $441(1)$ & $6834(1)$ & $19(1)$ \\
$\mathrm{C}(7)$ & $7389(2)$ & $991(1)$ & $6570(1)$ & $16(1)$ \\
$\mathrm{C}(8)$ & $4415(2)$ & $-180(1)$ & $7064(1)$ & $24(1)$ \\
$\mathrm{C}(9)$ & $4770(2)$ & $3373(1)$ & $5547(1)$ & $14(1)$ \\
$\mathrm{C}(10)$ & $3711(2)$ & $2912(1)$ & $5376(1)$ & $16(1)$ \\
$\mathrm{C}(11)$ & $2493(2)$ & $2912(1)$ & $5764(1)$ & $22(1)$ \\
$\mathrm{C}(12)$ & $2337(2)$ & $3371(1)$ & $6319(1)$ & $25(1)$ \\
$\mathrm{C}(13)$ & $3371(2)$ & $3838(1)$ & $6483(1)$ & $24(1)$
\end{tabular}




\begin{tabular}{lllll}
$\mathrm{C}(14)$ & $4591(2)$ & $3839(1)$ & $6102(1)$ & $19(1)$ \\
$\mathrm{C}(15)$ & $6060(2)$ & $3540(1)$ & $4155(1)$ & $13(1)$ \\
$\mathrm{C}(16)$ & $4730(2)$ & $3609(1)$ & $3864(1)$ & $19(1)$ \\
$\mathrm{C}(17)$ & $4529(2)$ & $3841(1)$ & $3168(1)$ & $27(1)$ \\
$\mathrm{C}(18)$ & $5657(2)$ & $3991(1)$ & $2750(1)$ & $29(1)$ \\
$\mathrm{C}(19)$ & $6989(2)$ & $3926(1)$ & $3037(1)$ & $23(1)$ \\
$\mathrm{C}(20)$ & $7194(2)$ & $3717(1)$ & $3739(1)$ & $16(1)$ \\
$\mathrm{C}(21)$ & $7469(2)$ & $4017(1)$ & $5379(1)$ & $14(1)$ \\
$\mathrm{C}(22)$ & $7046(2)$ & $4667(1)$ & $5169(1)$ & $20(1)$ \\
$\mathrm{C}(23)$ & $7835(2)$ & $5227(1)$ & $5357(1)$ & $25(1)$ \\
$\mathrm{C}(24)$ & $9056(2)$ & $5147(1)$ & $5758(1)$ & $27(1)$ \\
$\mathrm{C}(25)$ & $9488(2)$ & $4504(1)$ & $5959(1)$ & $24(1)$ \\
$\mathrm{C}(26)$ & $8708(2)$ & $3937(1)$ & $5772(1)$ & $17(1)$ \\
$\mathrm{C}(27)$ & $5931(2)$ & $1866(1)$ & $3888(1)$ & $12(1)$ \\
$\mathrm{C}(28)$ & $5699(2)$ & $2158(1)$ & $3214(1)$ & $16(1)$ \\
$\mathrm{C}(29)$ & $4442(2)$ & $2061(1)$ & $2843(1)$ & $21(1)$ \\
$\mathrm{C}(30)$ & $3396(2)$ & $1690(1)$ & $3147(1)$ & $23(1)$ \\
$\mathrm{C}(31)$ & $3596(2)$ & $1411(1)$ & $3825(1)$ & $21(1)$ \\
$\mathrm{C}(32)$ & $4865(2)$ & $1488(1)$ & $4189(1)$ & $17(1)$ \\
$\mathrm{C}(33)$ & $8834(2)$ & $2338(1)$ & $3888(1)$ & $11(1)$ \\
$\mathrm{C}(34)$ & $8970(2)$ & $2183(1)$ & $3162(1)$ & $16(1)$ \\
$\mathrm{C}(35)$ & $10007(2)$ & $2479(1)$ & $2767(1)$ & $21(1)$ \\
$\mathrm{C}(36)$ & $10950(2)$ & $2914(1)$ & $3094(1)$ & $23(1)$ \\
$\mathrm{C}(37)$ & $10875(2)$ & $3040(1)$ & $3823(1)$ & $19(1)$ \\
$\mathrm{C}(38)$ & $9826(2)$ & $2754(1)$ & $4222(1)$ & $15(1)$ \\
$\mathrm{C}(39)$ & $10011(2)$ & $1021(1)$ & $5252(1)$ & $23(1)$ \\
$\mathrm{C}(40)$ & $7270(2)$ & $302(1)$ & $4958(1)$ & $21(1)$ \\
$\mathrm{C}(41)$ & $9176(2)$ & $645(1)$ & $3802(1)$ & $26(1)$ \\
\hline
\end{tabular}

Table S6. Anisotropic displacement parameters $\left(\AA^{2} \mathrm{x} 10^{3}\right)$ for $\mathbf{1} \cdot \mathrm{Ni}(\mathrm{CO})_{3}$. The anisotropic displacement factor exponent takes the form: $-2 \pi^{2}\left[h^{2} a^{* 2} U^{11}+\ldots+2 h k a^{*} b^{*} U^{12}\right]$

\begin{tabular}{ccccccc}
\hline & $\mathrm{U}^{11}$ & $\mathrm{U}^{22}$ & $\mathrm{U}^{33}$ & $\mathrm{U}^{23}$ & $\mathrm{U}^{13}$ & $\mathrm{U}^{12}$ \\
\hline $\mathrm{Ni}(1)$ & $18(1)$ & $12(1)$ & $13(1)$ & $0(1)$ & $0(1)$ & $1(1)$ \\
$\mathrm{S}(1)$ & $11(1)$ & $14(1)$ & $6(1)$ & $0(1)$ & $-1(1)$ & $0(1)$ \\
$\mathrm{P}(1)$ & $10(1)$ & $12(1)$ & $10(1)$ & $-1(1)$ & $0(1)$ & $0(1)$ \\
$\mathrm{P}(2)$ & $12(1)$ & $11(1)$ & $7(1)$ & $0(1)$ & $0(1)$ & $-2(1)$ \\
$\mathrm{O}(1)$ & $16(1)$ & $16(1)$ & $9(1)$ & $-2(1)$ & $1(1)$ & $2(1)$ \\
$\mathrm{O}(2)$ & $12(1)$ & $19(1)$ & $13(1)$ & $-1(1)$ & $-3(1)$ & $2(1)$ \\
$\mathrm{O}(3)$ & $28(1)$ & $40(1)$ & $50(1)$ & $-18(1)$ & $-17(1)$ & $15(1)$ \\
$\mathrm{O}(4)$ & $44(1)$ & $27(1)$ & $34(1)$ & $2(1)$ & $6(1)$ & $-15(1)$ \\
$\mathrm{O}(5)$ & $74(1)$ & $36(1)$ & $30(1)$ & $-8(1)$ & $20(1)$ & $10(1)$ \\
$\mathrm{C}(1)$ & $11(1)$ & $12(1)$ & $7(1)$ & $-1(1)$ & $0(1)$ & $0(1)$ \\
$\mathrm{C}(2)$ & $16(1)$ & $15(1)$ & $6(1)$ & $1(1)$ & $0(1)$ & $0(1)$ \\
$\mathrm{C}(3)$ & $16(1)$ & $19(1)$ & $12(1)$ & $3(1)$ & $-1(1)$ & $0(1)$ \\
$\mathrm{C}(4)$ & $18(1)$ & $20(1)$ & $14(1)$ & $-1(1)$ & $1(1)$ & $-2(1)$ \\
$\mathrm{C}(5)$ & $27(1)$ & $16(1)$ & $9(1)$ & $-2(1)$ & $4(1)$ & $-2(1)$ \\
$\mathrm{C}(6)$ & $27(1)$ & $17(1)$ & $13(1)$ & $2(1)$ & $1(1)$ & $3(1)$ \\
$\mathrm{C}(7)$ & $18(1)$ & $20(1)$ & $11(1)$ & $1(1)$ & $-2(1)$ & $3(1)$ \\
$\mathrm{C}(8)$ & $34(1)$ & $16(1)$ & $23(1)$ & $1(1)$ & $9(1)$ & $-4(1)$ \\
$\mathrm{C}(9)$ & $12(1)$ & $19(1)$ & $12(1)$ & $2(1)$ & $0(1)$ & $3(1)$ \\
$\mathrm{C}(10)$ & $14(1)$ & $21(1)$ & $14(1)$ & $4(1)$ & $0(1)$ & $2(1)$ \\
$\mathrm{C}(11)$ & $13(1)$ & $28(1)$ & $25(1)$ & $12(1)$ & $0(1)$ & $1(1)$
\end{tabular}




$\begin{array}{lcccccc}\mathrm{C}(12) & 16(1) & 38(1) & 22(1) & 13(1) & 7(1) & 11(1) \\ \mathrm{C}(13) & 24(1) & 34(1) & 16(1) & 1(1) & 4(1) & 14(1) \\ \mathrm{C}(14) & 17(1) & 23(1) & 16(1) & -1(1) & 1(1) & 5(1) \\ \mathrm{C}(15) & 14(1) & 12(1) & 14(1) & 2(1) & 0(1) & 1(1) \\ \mathrm{C}(16) & 15(1) & 24(1) & 18(1) & 6(1) & -2(1) & -1(1) \\ \mathrm{C}(17) & 21(1) & 35(1) & 25(1) & 12(1) & -10(1) & -3(1) \\ \mathrm{C}(18) & 31(1) & 35(1) & 20(1) & 14(1) & -6(1) & -7(1) \\ \mathrm{C}(19) & 24(1) & 24(1) & 19(1) & 8(1) & 3(1) & -5(1) \\ \mathrm{C}(20) & 14(1) & 14(1) & 19(1) & 2(1) & 0(1) & 0(1) \\ \mathrm{C}(21) & 13(1) & 13(1) & 15(1) & -3(1) & 0(1) & -1(1) \\ \mathrm{C}(22) & 17(1) & 16(1) & 26(1) & -2(1) & -2(1) & 3(1) \\ \mathrm{C}(23) & 25(1) & 14(1) & 36(1) & -4(1) & -2(1) & 2(1) \\ \mathrm{C}(24) & 23(1) & 16(1) & 42(1) & -7(1) & -4(1) & -4(1) \\ \mathrm{C}(25) & 19(1) & 20(1) & 33(1) & -6(1) & -8(1) & -1(1) \\ \mathrm{C}(26) & 17(1) & 15(1) & 19(1) & -2(1) & -2(1) & 0(1) \\ \mathrm{C}(27) & 14(1) & 13(1) & 10(1) & -3(1) & -2(1) & 0(1) \\ \mathrm{C}(28) & 20(1) & 16(1) & 13(1) & 0(1) & -4(1) & -1(1) \\ \mathrm{C}(29) & 24(1) & 20(1) & 18(1) & -2(1) & -10(1) & 3(1) \\ \mathrm{C}(30) & 18(1) & 21(1) & 29(1) & -8(1) & -10(1) & 2(1) \\ \mathrm{C}(31) & 15(1) & 22(1) & 27(1) & -5(1) & 1(1) & -3(1) \\ \mathrm{C}(32) & 17(1) & 18(1) & 15(1) & -2(1) & 1(1) & -2(1) \\ \mathrm{C}(33) & 13(1) & 11(1) & 10(1) & 1(1) & 2(1) & 1(1) \\ \mathrm{C}(34) & 18(1) & 17(1) & 12(1) & -1(1) & 3(1) & 0(1) \\ \mathrm{C}(35) & 22(1) & 25(1) & 16(1) & 0(1) & 7(1) & 2(1) \\ \mathrm{C}(36) & 16(1) & 25(1) & 28(1) & 5(1) & 11(1) & 0(1) \\ \mathrm{C}(37) & 11(1) & 17(1) & 30(1) & -2(1) & 2(1) & -2(1) \\ \mathrm{C}(38) & 12(1) & 17(1) & 15(1) & -2(1) & 0(1) & 1(1) \\ \mathrm{C}(39) & 23(1) & 19(1) & 27(1) & -5(1) & -3(1) & 6(1) \\ \mathrm{C}(40) & 28(1) & 17(1) & 17(1) & -2(1) & -1(1) & 0(1) \\ \mathrm{C}(41) & 36(1) & 18(1) & 22(1) & 0(1) & 4(1) & 4(1) \\ & & & & & & \end{array}$




\subsection{Crystal Structure Determination of $1 \cdot \mathrm{Ni}(\mathrm{CO})_{2}$}

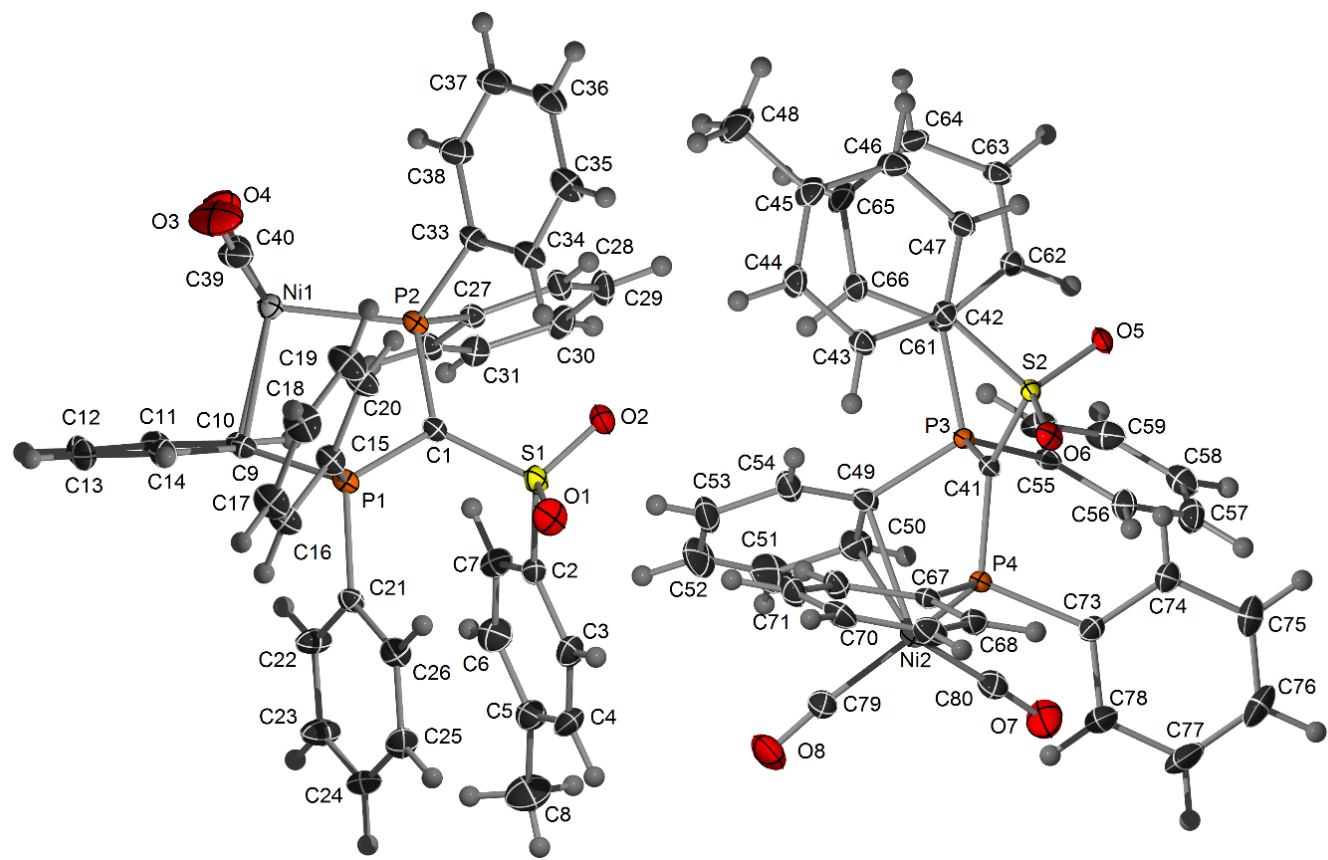

Figure S20. ORTEP Plot of $\mathbf{1}\left[\mathrm{Ni}(\mathrm{CO})_{2}\right]$. Ellipsoids are drawn at the $50 \%$ probability level.

Table S7. Atomic coordinates ( $\mathrm{x} 10^{4}$ ) and equivalent isotropic displacement parameters $\left(\AA^{2} \times 10^{3}\right)$ for $\mathbf{1} \cdot \mathrm{Ni}(\mathrm{CO})_{2}$. U(eq) is defined as one third of the trace of the orthogonalized $\mathrm{Uij}^{\mathrm{ij}}$ tensor.

\begin{tabular}{ccccc}
\hline & $\mathrm{x}$ & $\mathrm{y}$ & $\mathrm{z}$ & $\mathrm{U}(\mathrm{eq})$ \\
\hline $\mathrm{Ni}(1)$ & $3693(1)$ & $1617(1)$ & $6735(1)$ & $16(1)$ \\
$\mathrm{Ni}(2)$ & $1180(1)$ & $7336(1)$ & $3304(1)$ & $16(1)$ \\
$\mathrm{S}(1)$ & $3544(1)$ & $4570(1)$ & $5508(1)$ & $17(1)$ \\
$\mathrm{S}(2)$ & $1581(1)$ & $9512(1)$ & $4984(1)$ & $12(1)$ \\
$\mathrm{P}(1)$ & $4342(1)$ & $2754(1)$ & $5935(1)$ & $13(1)$ \\
$\mathrm{P}(2)$ & $3315(1)$ & $3145(1)$ & $6412(1)$ & $13(1)$ \\
$\mathrm{P}(3)$ & $688(1)$ & $7940(1)$ & $4284(1)$ & $11(1)$ \\
$\mathrm{P}(4)$ & $1673(1)$ & $8633(1)$ & $3855(1)$ & $13(1)$ \\
$\mathrm{O}(1)$ & $4117(1)$ & $5100(1)$ & $5543(1)$ & $27(1)$ \\
$\mathrm{O}(2)$ & $3064(1)$ & $5154(1)$ & $5636(1)$ & $24(1)$ \\
$\mathrm{O}(3)$ & $4735(1)$ & $1841(1)$ & $7795(1)$ & $45(1)$ \\
$\mathrm{O}(4)$ & $2667(1)$ & $406(1)$ & $6873(1)$ & $34(1)$ \\
$\mathrm{O}(5)$ & $1061(1)$ & $10118(1)$ & $5029(1)$ & $16(1)$ \\
$\mathrm{O}(6)$ & $2123(1)$ & $10064(1)$ & $4951(1)$ & $16(1)$ \\
$\mathrm{O}(7)$ & $232(1)$ & $8159(1)$ & $2299(1)$ & $40(1)$ \\
$\mathrm{O}(8)$ & $2092(1)$ & $5989(1)$ & $3057(1)$ & $39(1)$ \\
$\mathrm{C}(1)$ & $3705(1)$ & $3497(1)$ & $5928(1)$ & $14(1)$ \\
$\mathrm{C}(2)$ & $3228(1)$ & $4159(1)$ & $4790(1)$ & $17(1)$ \\
$\mathrm{C}(3)$ & $3422(1)$ & $4629(1)$ & $4373(1)$ & $26(1)$ \\
$\mathrm{C}(4)$ & $3169(1)$ & $4308(2)$ & $3813(1)$ & $31(1)$ \\
$\mathrm{C}(5)$ & $2734(1)$ & $3528(2)$ & $3662(1)$ & $29(1)$ \\
$\mathrm{C}(6)$ & $2545(1)$ & $3070(2)$ & $4084(1)$ & $27(1)$ \\
$\mathrm{C}(7)$ & $2787(1)$ & $3381(1)$ & $4645(1)$ & $22(1)$ \\
$\mathrm{C}(8)$ & $2467(1)$ & $3170(2)$ & $3054(1)$ & $50(1)$ \\
$\mathrm{C}(9)$ & $4221(1)$ & $1443(1)$ & $6117(1)$ & $14(1)$
\end{tabular}




\begin{tabular}{|c|c|c|c|c|}
\hline$C(10)$ & $3637(1)$ & $960(1)$ & $5868(1)$ & $14(1)$ \\
\hline$C(11)$ & $3597(1)$ & $-122(1)$ & $5865(1)$ & $18(1)$ \\
\hline$C(12)$ & $4120(1)$ & $-710(1)$ & $6097(1)$ & $22(1)$ \\
\hline$C(13)$ & $4702(1)$ & $-240(1)$ & $6347(1)$ & $21(1)$ \\
\hline$C(14)$ & $4755(1)$ & 808(1) & $6357(1)$ & $18(1)$ \\
\hline$C(15)$ & $5092(1)$ & $3150(1)$ & $6426(1)$ & $17(1)$ \\
\hline$C(16)$ & $5647(1)$ & 2885(1) & 6331(1) & $21(1)$ \\
\hline$C(17)$ & $6218(1)$ & 3158(1) & $6719(1)$ & $24(1)$ \\
\hline$C(18)$ & $6241(1)$ & $3690(2)$ & 7208(1) & $28(1)$ \\
\hline$C(19)$ & $5691(1)$ & $3956(2)$ & 7307(1) & $28(1)$ \\
\hline$C(20)$ & $5117(1)$ & 3691(1) & 6916(1) & $22(1)$ \\
\hline$C(21)$ & $4450(1)$ & $2706(1)$ & $5242(1)$ & $15(1)$ \\
\hline$C(22)$ & 4149(1) & 1949(1) & $4856(1)$ & $23(1)$ \\
\hline$C(23)$ & 4203(1) & 1923(2) & 4314(1) & $30(1)$ \\
\hline$C(24)$ & $4560(1)$ & 2647(1) & $4156(1)$ & $26(1)$ \\
\hline$C(25)$ & $4857(1)$ & 3403(1) & $4532(1)$ & $24(1)$ \\
\hline$C(26)$ & $4804(1)$ & $3440(1)$ & $5072(1)$ & $20(1)$ \\
\hline$C(27)$ & $2476(1)$ & $3266(1)$ & $6040(1)$ & $14(1)$ \\
\hline$C(28)$ & 2149(1) & $4168(1)$ & 6051(1) & $20(1)$ \\
\hline$C(29)$ & $1525(1)$ & $4256(1)$ & $5725(1)$ & $24(1)$ \\
\hline$C(30)$ & 1219(1) & $3459(1)$ & $5380(1)$ & $22(1)$ \\
\hline$C(31)$ & 1531(1) & 2547(1) & $5377(1)$ & $20(1)$ \\
\hline$C(32)$ & 2154(1) & 2449(1) & $5708(1)$ & $16(1)$ \\
\hline$C(33)$ & $3438(1)$ & 4103(1) & 6975(1) & $17(1)$ \\
\hline$C(34)$ & 3761(1) & $5022(1)$ & $7007(1)$ & $20(1)$ \\
\hline$C(35)$ & $3850(1)$ & $5671(1)$ & $7473(1)$ & $23(1)$ \\
\hline$C(36)$ & $3613(1)$ & $5415(1)$ & $7905(1)$ & $24(1)$ \\
\hline$C(37)$ & $3284(1)$ & $4512(2)$ & $7872(1)$ & $25(1)$ \\
\hline$C(38)$ & $3200(1)$ & $3858(1)$ & $7413(1)$ & $24(1)$ \\
\hline$C(39)$ & $4340(1)$ & $1742(2)$ & $7375(1)$ & $25(1)$ \\
\hline$C(40)$ & $3072(1)$ & 847(1) & $6808(1)$ & 21(1) \\
\hline$C(41)$ & 1346(1) & $8700(1)$ & 4413(1) & $12(1)$ \\
\hline$C(42)$ & $1820(1)$ & $8736(1)$ & $5605(1)$ & $14(1)$ \\
\hline$C(43)$ & $2259(1)$ & 7964(1) & $5655(1)$ & $16(1)$ \\
\hline$C(44)$ & $2420(1)$ & $7336(1)$ & 6131(1) & 21(1) \\
\hline$C(45)$ & 2151(1) & $7476(1)$ & $6555(1)$ & $22(1)$ \\
\hline$C(46)$ & $1728(1)$ & $8269(1)$ & $6502(1)$ & $22(1)$ \\
\hline $\mathrm{C}(47)$ & $1558(1)$ & 8902(1) & $6029(1)$ & $18(1)$ \\
\hline$C(48)$ & $2300(1)$ & $6767(2)$ & 7061(1) & $41(1)$ \\
\hline C(49) & $868(1)$ & $6730(1)$ & 4017(1) & $17(1)$ \\
\hline$C(50)$ & 453(1) & $6232(1)$ & 3543(1) & $26(1)$ \\
\hline$C(51)$ & $554(1)$ & $5192(2)$ & $3439(1)$ & $39(1)$ \\
\hline$C(52)$ & $1048(1)$ & $4672(2)$ & 3794(1) & $40(1)$ \\
\hline$C(53)$ & $1473(1)$ & $5156(1)$ & $4259(1)$ & $32(1)$ \\
\hline$C(54)$ & $1390(1)$ & $6171(1)$ & $4370(1)$ & $22(1)$ \\
\hline$C(55)$ & $-31(1)$ & $8442(1)$ & $3790(1)$ & $14(1)$ \\
\hline$C(56)$ & $-25(1)$ & $9332(1)$ & $3485(1)$ & $18(1)$ \\
\hline$C(57)$ & $-583(1)$ & $9748(1)$ & 3133(1) & $24(1)$ \\
\hline$C(58)$ & $-1144(1)$ & $9282(1)$ & $3089(1)$ & $24(1)$ \\
\hline$C(59)$ & $-1153(1)$ & $8389(2)$ & $3390(1)$ & $24(1)$ \\
\hline$C(60)$ & $-599(1)$ & 7968(1) & $3739(1)$ & 21(1) \\
\hline$C(61)$ & 477(1) & $7695(1)$ & 4920(1) & $14(1)$ \\
\hline$C(62)$ & 119(1) & $8424(1)$ & $5089(1)$ & $14(1)$ \\
\hline$C(63)$ & $-38(1)$ & $8269(1)$ & $5578(1)$ & $18(1)$ \\
\hline$C(64)$ & $147(1)$ & $7387(2)$ & $5895(1)$ & $24(1)$ \\
\hline
\end{tabular}




\begin{tabular}{lrrrr}
$\mathrm{C}(65)$ & $491(1)$ & $6653(2)$ & $5725(1)$ & $27(1)$ \\
$\mathrm{C}(66)$ & $658(1)$ & $6804(1)$ & $5240(1)$ & $21(1)$ \\
$\mathrm{C}(67)$ & $2523(1)$ & $8558(1)$ & $4170(1)$ & $15(1)$ \\
$\mathrm{C}(68)$ & $2917(1)$ & $9387(1)$ & $4194(1)$ & $19(1)$ \\
$\mathrm{C}(69)$ & $3557(1)$ & $9286(1)$ & $4450(1)$ & $22(1)$ \\
$\mathrm{C}(70)$ & $3811(1)$ & $8367(1)$ & $4703(1)$ & $21(1)$ \\
$\mathrm{C}(71)$ & $3425(1)$ & $7535(1)$ & $4680(1)$ & $22(1)$ \\
$\mathrm{C}(72)$ & $2786(1)$ & $7624(1)$ & $4405(1)$ & $19(1)$ \\
$\mathrm{C}(73)$ & $1535(1)$ & $9854(1)$ & $3464(1)$ & $17(1)$ \\
$\mathrm{C}(74)$ & $1312(1)$ & $10732(1)$ & $3652(1)$ & $19(1)$ \\
$\mathrm{C}(75)$ & $1109(1)$ & $11565(1)$ & $3292(1)$ & $27(1)$ \\
$\mathrm{C}(76)$ & $1144(1)$ & $11548(2)$ & $2746(1)$ & $34(1)$ \\
$\mathrm{C}(77)$ & $1394(1)$ & $10704(2)$ & $2564(1)$ & $41(1)$ \\
$\mathrm{C}(78)$ & $1582(1)$ & $9860(2)$ & $2919(1)$ & $32(1)$ \\
$\mathrm{C}(79)$ & $1737(1)$ & $6485(1)$ & $3170(1)$ & $23(1)$ \\
$\mathrm{C}(80)$ & $590(1)$ & $7826(1)$ & $2698(1)$ & $24(1)$ \\
\hline
\end{tabular}

Table S8. Anisotropic displacement parameters $\left(\AA^{2} \mathrm{x} 10^{3}\right)$ for $\mathbf{1} \cdot \mathrm{Ni}(\mathrm{CO})_{2}$. The anisotropic displacement factor exponent takes the form: $-2 \pi^{2}\left[h^{2} a^{* 2} U^{11}+\ldots+2 h \mathrm{k} \mathrm{a}^{*} \mathrm{~b}^{*} \mathrm{U}^{12}\right]$

\begin{tabular}{|c|c|c|c|c|c|c|}
\hline & $\mathrm{U}^{11}$ & $\mathrm{U}^{22}$ & $\mathrm{U}^{33}$ & $\mathrm{U}^{23}$ & $\mathrm{U}^{13}$ & $\mathrm{U}^{12}$ \\
\hline $\mathrm{Ni}(1)$ & $15(1)$ & $18(1)$ & $15(1)$ & $1(1)$ & $5(1)$ & $2(1)$ \\
\hline $\mathrm{Ni}(2)$ & $22(1)$ & $15(1)$ & $13(1)$ & $-3(1)$ & $6(1)$ & $2(1)$ \\
\hline $\mathrm{S}(1)$ & $20(1)$ & $11(1)$ & $23(1)$ & $-1(1)$ & $10(1)$ & $-2(1)$ \\
\hline $\mathrm{S}(2)$ & $12(1)$ & $11(1)$ & $12(1)$ & $-2(1)$ & $3(1)$ & $2(1)$ \\
\hline $\mathrm{P}(1)$ & $11(1)$ & $14(1)$ & $14(1)$ & $-3(1)$ & $5(1)$ & $-2(1)$ \\
\hline $\mathrm{P}(2)$ & $13(1)$ & $14(1)$ & $14(1)$ & $-3(1)$ & $6(1)$ & $0(1)$ \\
\hline $\mathrm{P}(3)$ & $12(1)$ & $11(1)$ & $11(1)$ & $-1(1)$ & $4(1)$ & 1(1) \\
\hline $\mathrm{P}(4)$ & $16(1)$ & $12(1)$ & $13(1)$ & $0(1)$ & $7(1)$ & $2(1)$ \\
\hline $\mathrm{O}(1)$ & $28(1)$ & $19(1)$ & $37(1)$ & $-4(1)$ & $15(1)$ & $-11(1)$ \\
\hline $\mathrm{O}(2)$ & $31(1)$ & $16(1)$ & $29(1)$ & 1(1) & $16(1)$ & $6(1)$ \\
\hline $\mathrm{O}(3)$ & $35(1)$ & $74(1)$ & $19(1)$ & $-4(1)$ & $0(1)$ & $0(1)$ \\
\hline $\mathrm{O}(4)$ & $30(1)$ & $36(1)$ & $39(1)$ & $5(1)$ & $16(1)$ & $-6(1)$ \\
\hline $\mathrm{O}(5)$ & $17(1)$ & $14(1)$ & $17(1)$ & $-2(1)$ & $5(1)$ & $6(1)$ \\
\hline $\mathrm{O}(6)$ & $16(1)$ & $16(1)$ & $18(1)$ & $-3(1)$ & $6(1)$ & $-3(1)$ \\
\hline $\mathrm{O}(7)$ & $50(1)$ & $40(1)$ & $20(1)$ & $-1(1)$ & $-2(1)$ & $14(1)$ \\
\hline $\mathrm{O}(8)$ & $43(1)$ & $41(1)$ & $35(1)$ & $-3(1)$ & $16(1)$ & $21(1)$ \\
\hline $\mathrm{C}(1)$ & $14(1)$ & $14(1)$ & $16(1)$ & $-2(1)$ & $6(1)$ & $0(1)$ \\
\hline $\mathrm{C}(2)$ & $18(1)$ & $16(1)$ & $19(1)$ & $3(1)$ & $8(1)$ & $4(1)$ \\
\hline $\mathrm{C}(3)$ & $33(1)$ & $18(1)$ & $32(1)$ & $6(1)$ & $18(1)$ & $2(1)$ \\
\hline $\mathrm{C}(4)$ & $43(1)$ & $32(1)$ & $24(1)$ & $15(1)$ & $18(1)$ & $13(1)$ \\
\hline $\mathrm{C}(5)$ & $32(1)$ & $36(1)$ & $18(1)$ & $6(1)$ & $6(1)$ & $16(1)$ \\
\hline$C(6)$ & $22(1)$ & $33(1)$ & $22(1)$ & $0(1)$ & $2(1)$ & $2(1)$ \\
\hline$C(7)$ & $20(1)$ & $26(1)$ & $20(1)$ & $3(1)$ & $7(1)$ & $-1(1)$ \\
\hline $\mathrm{C}(8)$ & $64(2)$ & $60(2)$ & $19(1)$ & $3(1)$ & $6(1)$ & $12(1)$ \\
\hline$C(9)$ & $13(1)$ & $16(1)$ & $14(1)$ & $-1(1)$ & $5(1)$ & $0(1)$ \\
\hline$C(10)$ & $13(1)$ & $17(1)$ & $14(1)$ & $-1(1)$ & $5(1)$ & $0(1)$ \\
\hline$C(11)$ & $20(1)$ & $18(1)$ & $18(1)$ & $-4(1)$ & $7(1)$ & $-4(1)$ \\
\hline$C(12)$ & $28(1)$ & $15(1)$ & $23(1)$ & $-1(1)$ & $10(1)$ & 1(1) \\
\hline$C(13)$ & $20(1)$ & $22(1)$ & $22(1)$ & $3(1)$ & $6(1)$ & $7(1)$ \\
\hline$C(14)$ & $14(1)$ & $22(1)$ & $18(1)$ & $0(1)$ & $4(1)$ & $2(1)$ \\
\hline$C(15)$ & $14(1)$ & $18(1)$ & $20(1)$ & $-4(1)$ & $4(1)$ & $-3(1)$ \\
\hline$C(16)$ & $15(1)$ & $23(1)$ & $24(1)$ & $-6(1)$ & $7(1)$ & $-3(1)$ \\
\hline
\end{tabular}




\begin{tabular}{|c|c|c|c|c|c|c|}
\hline $\mathrm{C}(17)$ & $13(1)$ & $29(1)$ & $30(1)$ & $-4(1)$ & $6(1)$ & $-4(1)$ \\
\hline$C(18)$ & $18(1)$ & $30(1)$ & $30(1)$ & $-8(1)$ & $0(1)$ & $-7(1)$ \\
\hline$C(19)$ & $24(1)$ & $31(1)$ & $26(1)$ & $-14(1)$ & $4(1)$ & $-5(1)$ \\
\hline $\mathrm{C}(20)$ & $18(1)$ & $26(1)$ & $24(1)$ & $-9(1)$ & $7(1)$ & $-2(1)$ \\
\hline $\mathrm{C}(21)$ & $14(1)$ & $16(1)$ & $17(1)$ & $-1(1)$ & $7(1)$ & $0(1)$ \\
\hline $\mathrm{C}(22)$ & $32(1)$ & 21(1) & $20(1)$ & $-4(1)$ & $14(1)$ & $-12(1)$ \\
\hline $\mathrm{C}(23)$ & $46(1)$ & $26(1)$ & 21(1) & $-7(1)$ & $17(1)$ & $-12(1)$ \\
\hline $\mathrm{C}(24)$ & $34(1)$ & $28(1)$ & $23(1)$ & $3(1)$ & $18(1)$ & 1(1) \\
\hline$C(25)$ & 21(1) & $24(1)$ & $32(1)$ & $5(1)$ & $16(1)$ & $-2(1)$ \\
\hline$C(26)$ & $16(1)$ & 19(1) & $27(1)$ & $-1(1)$ & $10(1)$ & $-4(1)$ \\
\hline $\mathrm{C}(27)$ & $14(1)$ & $14(1)$ & $17(1)$ & $0(1)$ & $7(1)$ & $0(1)$ \\
\hline $\mathrm{C}(28)$ & 21(1) & $15(1)$ & $27(1)$ & $0(1)$ & $10(1)$ & $3(1)$ \\
\hline$C(29)$ & $21(1)$ & $18(1)$ & $34(1)$ & $6(1)$ & $11(1)$ & $8(1)$ \\
\hline$C(30)$ & $14(1)$ & $25(1)$ & $27(1)$ & $9(1)$ & $5(1)$ & $4(1)$ \\
\hline $\mathrm{C}(31)$ & $14(1)$ & $20(1)$ & $24(1)$ & $1(1)$ & $6(1)$ & $-2(1)$ \\
\hline$C(32)$ & $14(1)$ & $14(1)$ & $22(1)$ & $0(1)$ & $8(1)$ & $0(1)$ \\
\hline $\mathrm{C}(33)$ & $16(1)$ & $20(1)$ & $16(1)$ & $-4(1)$ & $5(1)$ & 1(1) \\
\hline $\mathrm{C}(34)$ & $16(1)$ & $23(1)$ & $20(1)$ & $-6(1)$ & $6(1)$ & $0(1)$ \\
\hline$C(35)$ & $17(1)$ & $24(1)$ & $25(1)$ & $-10(1)$ & $3(1)$ & $-4(1)$ \\
\hline$C(36)$ & 21(1) & $30(1)$ & $18(1)$ & $-10(1)$ & 1(1) & $4(1)$ \\
\hline$C(37)$ & $28(1)$ & $32(1)$ & $18(1)$ & $-3(1)$ & $10(1)$ & $4(1)$ \\
\hline $\mathrm{C}(38)$ & $29(1)$ & $25(1)$ & 21(1) & $-4(1)$ & $11(1)$ & $-3(1)$ \\
\hline C(39) & $25(1)$ & $34(1)$ & 19(1) & $0(1)$ & $10(1)$ & $3(1)$ \\
\hline $\mathrm{C}(40)$ & $24(1)$ & 21(1) & 19(1) & $2(1)$ & $8(1)$ & $3(1)$ \\
\hline $\mathrm{C}(41)$ & $12(1)$ & $12(1)$ & $12(1)$ & $-2(1)$ & $4(1)$ & $1(1)$ \\
\hline$C(42)$ & $12(1)$ & $15(1)$ & $12(1)$ & $-1(1)$ & 1(1) & $2(1)$ \\
\hline$C(43)$ & $13(1)$ & $19(1)$ & $18(1)$ & $-1(1)$ & $5(1)$ & $3(1)$ \\
\hline$C(44)$ & $17(1)$ & $20(1)$ & $23(1)$ & $5(1)$ & $4(1)$ & $6(1)$ \\
\hline$C(45)$ & $21(1)$ & $27(1)$ & $17(1)$ & $6(1)$ & $3(1)$ & $4(1)$ \\
\hline$C(46)$ & $21(1)$ & $31(1)$ & $13(1)$ & 1(1) & $5(1)$ & $5(1)$ \\
\hline $\mathrm{C}(47)$ & $16(1)$ & $22(1)$ & $14(1)$ & $-1(1)$ & $3(1)$ & $5(1)$ \\
\hline $\mathrm{C}(48)$ & $44(1)$ & $49(1)$ & $32(1)$ & $24(1)$ & $16(1)$ & $18(1)$ \\
\hline $\mathrm{C}(49)$ & $22(1)$ & $13(1)$ & $20(1)$ & $-4(1)$ & $12(1)$ & $-2(1)$ \\
\hline$C(50)$ & $35(1)$ & $21(1)$ & $23(1)$ & $-6(1)$ & $9(1)$ & $-6(1)$ \\
\hline $\mathrm{C}(51)$ & $54(1)$ & $25(1)$ & $41(1)$ & $-17(1)$ & $19(1)$ & $-15(1)$ \\
\hline$C(52)$ & $65(2)$ & $12(1)$ & $56(1)$ & $-6(1)$ & $36(1)$ & $-3(1)$ \\
\hline$C(53)$ & $42(1)$ & $16(1)$ & $48(1)$ & $5(1)$ & $28(1)$ & $9(1)$ \\
\hline$C(54)$ & $23(1)$ & $16(1)$ & $31(1)$ & 1(1) & $14(1)$ & $2(1)$ \\
\hline$C(55)$ & $13(1)$ & $19(1)$ & $10(1)$ & $-2(1)$ & $3(1)$ & $2(1)$ \\
\hline$C(56)$ & $15(1)$ & $18(1)$ & $21(1)$ & 1(1) & $3(1)$ & $2(1)$ \\
\hline $\mathrm{C}(57)$ & $24(1)$ & $21(1)$ & $23(1)$ & $3(1)$ & $3(1)$ & $6(1)$ \\
\hline $\mathrm{C}(58)$ & $16(1)$ & $33(1)$ & $18(1)$ & $-6(1)$ & $-1(1)$ & $8(1)$ \\
\hline $\mathrm{C}(59)$ & $14(1)$ & $39(1)$ & $18(1)$ & $-4(1)$ & $3(1)$ & $-2(1)$ \\
\hline $\mathrm{C}(60)$ & $17(1)$ & $30(1)$ & $15(1)$ & 1(1) & $5(1)$ & $-3(1)$ \\
\hline$C(61)$ & $11(1)$ & $17(1)$ & $13(1)$ & 1(1) & $4(1)$ & 1(1) \\
\hline$C(62)$ & $12(1)$ & $17(1)$ & $13(1)$ & $0(1)$ & $2(1)$ & $2(1)$ \\
\hline$C(63)$ & $14(1)$ & $25(1)$ & $15(1)$ & $0(1)$ & $6(1)$ & $5(1)$ \\
\hline$C(64)$ & $22(1)$ & $38(1)$ & $16(1)$ & $10(1)$ & $10(1)$ & $9(1)$ \\
\hline$C(65)$ & $29(1)$ & $31(1)$ & $26(1)$ & $17(1)$ & $14(1)$ & $15(1)$ \\
\hline$C(66)$ & $20(1)$ & $22(1)$ & $24(1)$ & $8(1)$ & 11(1) & 11(1) \\
\hline $\mathrm{C}(67)$ & $17(1)$ & $16(1)$ & $16(1)$ & $-1(1)$ & $10(1)$ & 2(1) \\
\hline $\mathrm{C}(68)$ & $22(1)$ & $17(1)$ & 21(1) & $1(1)$ & $12(1)$ & 1(1) \\
\hline C(69) & 21(1) & $22(1)$ & $28(1)$ & $-5(1)$ & $13(1)$ & $-5(1)$ \\
\hline$C(70)$ & $16(1)$ & $26(1)$ & $24(1)$ & $-6(1)$ & $9(1)$ & $2(1)$ \\
\hline $\mathrm{C}(71)$ & $20(1)$ & $20(1)$ & $27(1)$ & $0(1)$ & $10(1)$ & $6(1)$ \\
\hline
\end{tabular}




\begin{tabular}{lcccccc}
$\mathrm{C}(72)$ & $18(1)$ & $16(1)$ & $25(1)$ & $0(1)$ & $10(1)$ & $1(1)$ \\
$\mathrm{C}(73)$ & $19(1)$ & $17(1)$ & $17(1)$ & $4(1)$ & $7(1)$ & $1(1)$ \\
$\mathrm{C}(74)$ & $20(1)$ & $15(1)$ & $21(1)$ & $2(1)$ & $6(1)$ & $-1(1)$ \\
$\mathrm{C}(75)$ & $28(1)$ & $16(1)$ & $34(1)$ & $6(1)$ & $6(1)$ & $0(1)$ \\
$\mathrm{C}(76)$ & $41(1)$ & $25(1)$ & $32(1)$ & $16(1)$ & $6(1)$ & $-1(1)$ \\
$\mathrm{C}(77)$ & $65(2)$ & $39(1)$ & $24(1)$ & $14(1)$ & $20(1)$ & $1(1)$ \\
$\mathrm{C}(78)$ & $51(1)$ & $27(1)$ & $24(1)$ & $6(1)$ & $21(1)$ & $4(1)$ \\
$\mathrm{C}(79)$ & $31(1)$ & $20(1)$ & $17(1)$ & $-2(1)$ & $7(1)$ & $4(1)$ \\
$\mathrm{C}(80)$ & $31(1)$ & $21(1)$ & $18(1)$ & $-5(1)$ & $7(1)$ & $2(1)$ \\
\hline
\end{tabular}

\subsection{Crystal Structure Determination of $1 \cdot \mathrm{Pd}(\mathrm{allyl}) \mathrm{Cl}$}

There are two independent molecules in the unit cell. Both allyl groups bound to palladium were disordered over two positions and modelled using appropriate PART, SAME, SIMU, RIGU and ISOR instructions. The final occupancy of the allyl groups was 0.69:0.31 and 0.73:0.27. Additionally both DCM molecules were only partially occupied. The final occupancy was 0.87 and 0.89 .

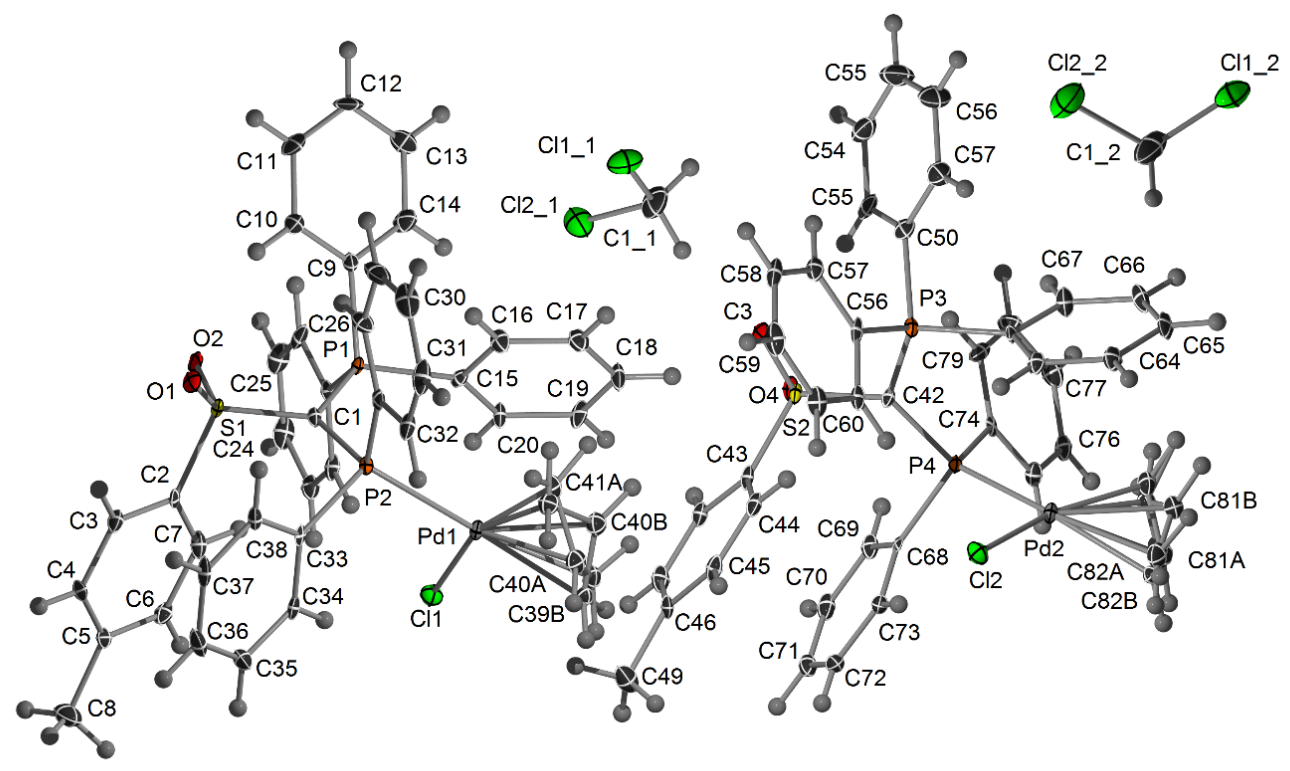

Figure S21. ORTEP Plot of 1·Pd(allyl)Cl. Ellipsoids are drawn at the $50 \%$ probability level.

Table S9. Atomic coordinates ( $\left.\mathrm{x} 10^{4}\right)$ and equivalent isotropic displacement parameters $\left(\AA^{2} \mathrm{x} 10^{3}\right)$ for $1 \cdot \operatorname{Pd}($ allyl $) \mathrm{Cl}$. $\mathrm{U}(\mathrm{eq})$ is defined as one third of the trace of the orthogonalized $\mathrm{U}^{\mathrm{ij}}$ tensor.

\begin{tabular}{ccccc}
\hline & $\mathrm{x}$ & $\mathrm{y}$ & $\mathrm{z}$ & $\mathrm{U}(\mathrm{eq})$ \\
\hline $\mathrm{Pd}(1)$ & $5072(1)$ & $5886(1)$ & $3679(1)$ & $9(1)$ \\
$\mathrm{Pd}(2)$ & $409(1)$ & $889(1)$ & $3632(1)$ & $10(1)$ \\
$\mathrm{Cl}(1)$ & $3203(1)$ & $6274(1)$ & $4096(1)$ & $15(1)$ \\
$\mathrm{Cl}(2)$ & $2082(1)$ & $1248(1)$ & $4102(1)$ & $15(1)$ \\
$\mathrm{S}(1)$ & $5582(1)$ & $8850(1)$ & $2303(1)$ & $9(1)$ \\
$\mathrm{S}(2)$ & $310(1)$ & $3866(1)$ & $2229(1)$ & $10(1)$ \\
$\mathrm{P}(1)$ & $4249(1)$ & $7806(1)$ & $1905(1)$ & $7(1)$
\end{tabular}




\begin{tabular}{|c|c|c|c|c|}
\hline $\mathrm{P}(2)$ & $6104(1)$ & $7107(1)$ & $3046(1)$ & $7(1)$ \\
\hline $\mathrm{P}(3)$ & 1831(1) & 2835(1) & $1838(1)$ & $9(1)$ \\
\hline $\mathrm{P}(4)$ & $-412(1)$ & 2106(1) & 2996(1) & $8(1)$ \\
\hline $\mathrm{O}(1)$ & 6884(2) & $9056(2)$ & $2144(2)$ & $13(1)$ \\
\hline $\mathrm{O}(2)$ & $4779(3)$ & 9336(2) & $1775(2)$ & $13(1)$ \\
\hline $\mathrm{O}(3)$ & 1297(3) & $4346(2)$ & $1699(2)$ & $14(1)$ \\
\hline $\mathrm{O}(4)$ & $-941(2)$ & 4073(2) & $2065(2)$ & $12(1)$ \\
\hline $\mathrm{C}(1)$ & $5274(3)$ & $7900(2)$ & $2455(2)$ & $9(1)$ \\
\hline$C(2)$ & 5113(4) & $8955(2)$ & $3093(2)$ & $9(1)$ \\
\hline$C(3)$ & $5878(4)$ & 9354(2) & $3325(2)$ & $12(1)$ \\
\hline$C(4)$ & $5480(4)$ & $9463(2)$ & $3927(2)$ & $13(1)$ \\
\hline$C(5)$ & $4334(4)$ & $9167(2)$ & $4300(2)$ & $14(1)$ \\
\hline$C(6)$ & 3582(4) & $8770(2)$ & $4054(2)$ & $13(1)$ \\
\hline$C(7)$ & $3962(4)$ & $8663(2)$ & $3458(2)$ & $12(1)$ \\
\hline$C(8)$ & $3895(4)$ & 9255(3) & $4974(2)$ & $25(1)$ \\
\hline$C(9)$ & $4742(4)$ & $8236(2)$ & $945(2)$ & $10(1)$ \\
\hline$C(10)$ & $5625(4)$ & $8839(2)$ & $603(2)$ & $16(1)$ \\
\hline $\mathrm{C}(11)$ & 5935(4) & 9133(3) & $-136(2)$ & $24(1)$ \\
\hline$C(12)$ & $5367(4)$ & $8825(3)$ & $-527(2)$ & $23(1)$ \\
\hline$C(13)$ & $4480(5)$ & 8234(3) & $-189(2)$ & $25(1)$ \\
\hline$C(14)$ & 4158(4) & 7936(3) & $547(2)$ & $19(1)$ \\
\hline$C(15)$ & 4055(4) & $6780(2)$ & $2106(2)$ & $9(1)$ \\
\hline$C(16)$ & $5004(4)$ & $6430(3)$ & $1872(2)$ & $15(1)$ \\
\hline$C(17)$ & $4824(4)$ & $5686(3)$ & $1924(2)$ & $15(1)$ \\
\hline $\mathrm{C}(18)$ & $3697(4)$ & $5283(3)$ & $2210(2)$ & $17(1)$ \\
\hline $\mathrm{C}(19)$ & 2749(4) & $5620(2)$ & $2448(2)$ & $15(1)$ \\
\hline$C(20)$ & 2925(4) & $6367(2)$ & $2399(2)$ & $12(1)$ \\
\hline $\mathrm{C}(21)$ & 2732(4) & $8168(2)$ & 1983(2) & $9(1)$ \\
\hline $\mathrm{C}(22)$ & 2067(4) & 7887(2) & 2663(2) & $12(1)$ \\
\hline $\mathrm{C}(23)$ & $902(4)$ & $8150(2)$ & $2730(2)$ & $14(1)$ \\
\hline $\mathrm{C}(24)$ & $386(4)$ & $8686(3)$ & 2134(3) & $19(1)$ \\
\hline $\mathrm{C}(25)$ & 1034(4) & 8966(3) & 1467(3) & $18(1)$ \\
\hline$C(26)$ & 2207(4) & $8715(2)$ & $1386(2)$ & $15(1)$ \\
\hline $\mathrm{C}(27)$ & 7578(4) & 7087(2) & $2555(2)$ & $10(1)$ \\
\hline $\mathrm{C}(28)$ & 7803(4) & $7525(3)$ & $1818(2)$ & $15(1)$ \\
\hline C(29) & 8911(4) & 7457(3) & $1457(3)$ & $23(1)$ \\
\hline $\mathrm{C}(30)$ & $9785(4)$ & 6958(3) & $1829(3)$ & $24(1)$ \\
\hline $\mathrm{C}(31)$ & $9565(4)$ & $6532(3)$ & $2565(3)$ & 21(1) \\
\hline $\mathrm{C}(32)$ & $8470(4)$ & 6597(2) & 2923(2) & $15(1)$ \\
\hline$C(33)$ & $6592(4)$ & 7414(2) & $3699(2)$ & $8(1)$ \\
\hline$C(34)$ & 5793(4) & $7276(2)$ & $4318(2)$ & $11(1)$ \\
\hline$C(35)$ & $6147(4)$ & $7523(2)$ & $4807(2)$ & $16(1)$ \\
\hline$C(36)$ & 7291(4) & 7898(3) & $4704(2)$ & 19(1) \\
\hline $\mathrm{C}(37)$ & $8088(4)$ & $8033(2)$ & $4100(2)$ & $16(1)$ \\
\hline $\mathrm{C}(38)$ & $7739(4)$ & $7795(2)$ & $3597(2)$ & $12(1)$ \\
\hline$C(39 A)$ & $4461(6)$ & $4646(5)$ & $4186(4)$ & $15(2)$ \\
\hline$C(40 A)$ & $5737(5)$ & $4718(4)$ & $4137(3)$ & $16(1)$ \\
\hline$C(41 \mathrm{~A})$ & $6372(7)$ & $5169(5)$ & $3447(4)$ & $14(2)$ \\
\hline$C(39 B)$ & $4599(14)$ & $4630(10)$ & $4387(8)$ & $12(3)$ \\
\hline$C(40 B)$ & $5298(11)$ & $4722(8)$ & $3753(7)$ & $15(2)$ \\
\hline$C(41 B)$ & $6444(14)$ & $5137(12)$ & $3623(10)$ & $14(3)$ \\
\hline $\mathrm{C}(42)$ & $590(4)$ & $2916(2)$ & $2381(2)$ & $9(1)$ \\
\hline $\mathrm{C}(43)$ & $501(4)$ & $3976(2)$ & $3014(2)$ & $11(1)$ \\
\hline$C(44)$ & 1527(4) & $3684(2)$ & $3373(2)$ & $13(1)$ \\
\hline $\mathrm{C}(45)$ & $1726(4)$ & $3795(2)$ & $3968(2)$ & $13(1)$ \\
\hline
\end{tabular}




\begin{tabular}{ccccc}
$\mathrm{C}(46)$ & $883(4)$ & $4192(2)$ & $4220(2)$ & $13(1)$ \\
$\mathrm{C}(47)$ & $-134(4)$ & $4484(2)$ & $3852(2)$ & $15(1)$ \\
$\mathrm{C}(48)$ & $-334(4)$ & $4383(2)$ & $3248(2)$ & $12(1)$ \\
$\mathrm{C}(49)$ & $1068(4)$ & $4278(3)$ & $4891(2)$ & $22(1)$ \\
$\mathrm{C}(50)$ & $1730(4)$ & $3310(2)$ & $873(2)$ & $14(1)$ \\
$\mathrm{C}(51)$ & $2595(4)$ & $3100(3)$ & $453(2)$ & $20(1)$ \\
$\mathrm{C}(52)$ & $2581(5)$ & $3456(3)$ & $-297(3)$ & $29(1)$ \\
$\mathrm{C}(53)$ & $1736(5)$ & $4005(3)$ & $-617(3)$ & $33(1)$ \\
$\mathrm{C}(54)$ & $878(5)$ & $4203(3)$ & $-201(2)$ & $24(1)$ \\
$\mathrm{C}(55)$ & $865(4)$ & $3857(2)$ & $543(2)$ & $17(1)$ \\
$\mathrm{C}(56)$ & $3305(4)$ & $3183(2)$ & $1958(2)$ & $12(1)$ \\
$\mathrm{C}(57)$ & $4040(4)$ & $3762(2)$ & $1388(2)$ & $14(1)$ \\
$\mathrm{C}(58)$ & $5164(4)$ & $4010(2)$ & $1494(3)$ & $17(1)$ \\
$\mathrm{C}(59)$ & $5570(4)$ & $3692(3)$ & $2164(3)$ & $18(1)$ \\
$\mathrm{C}(60)$ & $4836(4)$ & $3116(3)$ & $2742(2)$ & $16(1)$ \\
$\mathrm{C}(61)$ & $3713(4)$ & $2861(2)$ & $2638(2)$ & $12(1)$ \\
$\mathrm{C}(62)$ & $1973(4)$ & $1819(2)$ & $2003(2)$ & $10(1)$ \\
$\mathrm{C}(63)$ & $2881(4)$ & $1355(2)$ & $2397(2)$ & $11(1)$ \\
$\mathrm{C}(64)$ & $3043(4)$ & $610(2)$ & $2441(2)$ & $15(1)$ \\
$\mathrm{C}(65)$ & $2300(4)$ & $315(3)$ & $2104(2)$ & $16(1)$ \\
$\mathrm{C}(66)$ & $1371(4)$ & $768(3)$ & $1734(2)$ & $18(1)$ \\
$\mathrm{C}(67)$ & $1195(4)$ & $1514(2)$ & $1681(2)$ & $16(1)$ \\
$\mathrm{C}(68)$ & $-1121(4)$ & $2424(2)$ & $3649(2)$ & $9(1)$ \\
$\mathrm{C}(69)$ & $-2236(4)$ & $2809(2)$ & $3547(2)$ & $13(1)$ \\
$\mathrm{C}(70)$ & $-2759(4)$ & $3052(2)$ & $4039(2)$ & $17(1)$ \\
$\mathrm{C}(71)$ & $-2175(4)$ & $2929(2)$ & $4639(2)$ & $17(1)$ \\
$\mathrm{C}(72)$ & $-1069(4)$ & $2550(2)$ & $4745(2)$ & $16(1)$ \\
$\mathrm{C}(73)$ & $-552(4)$ & $2293(2)$ & $4264(2)$ & $12(1)$ \\
$\mathrm{C}(74)$ & $-1753(4)$ & $2060(2)$ & $2539(2)$ & $9(1)$ \\
$\mathrm{C}(75)$ & $-2760(4)$ & $1566(2)$ & $2953(2)$ & $12(1)$ \\
$\mathrm{C}(76)$ & $-3784(4)$ & $1500(2)$ & $2633(2)$ & $13(1)$ \\
$\mathrm{C}(77)$ & $-3801(4)$ & $1912(3)$ & $1899(2)$ & $19(1)$ \\
$\mathrm{C}(78)$ & $-2800(4)$ & $2402(3)$ & $1484(2)$ & $17(1)$ \\
$\mathrm{C}(79)$ & $-1780(4)$ & $2477(2)$ & $1814(2)$ & $14(1)$ \\
$\mathrm{C}(80 \mathrm{~A})$ & $-721(7)$ & $200(5)$ & $3342(4)$ & $16(2)$ \\
$\mathrm{C}(81 \mathrm{~A})$ & $-451(5)$ & $-271(3)$ & $4062(3)$ & $18(1)$ \\
$\mathrm{C}(82 \mathrm{~A})$ & $786(6)$ & $-363(5)$ & $4171(4)$ & $18(2)$ \\
$\mathrm{C}(80 \mathrm{~B})$ & $-898(18)$ & $152(14)$ & $3490(11)$ & $12(3)$ \\
$\mathrm{C}(81 \mathrm{~B})$ & $194(13)$ & $-261(9)$ & $3672(8)$ & $14(3)$ \\
$\mathrm{C}(82 \mathrm{~B})$ & $564(18)$ & $-364(12)$ & $4336(8)$ & $10(3)$ \\
$\mathrm{C} 111$ & $4274(1)$ & $6022(1)$ & $191(1)$ & $29(1)$ \\
$\mathrm{C} 121$ & $1850(1)$ & $6500(1)$ & $512(1)$ & $32(2)$ \\
$\mathrm{C} 11$ & $2724(5)$ & $5742(3)$ & $471(3)$ & $42(1)$ \\
$\mathrm{C} 112$ & $8405(1)$ & $-94(1)$ & $490(1)$ & $436(1)$ \\
$\mathrm{C} 122$ & $8882(2)$ & $1436(1)$ & $893(3)$ & \\
$\mathrm{C} 12$ & $8037(6)$ & $534(3)$ & & \\
\hline & & & & \\
\end{tabular}

Table S10. Anisotropic displacement parameters $\left(\AA^{2} \times 10^{3}\right)$ for $\mathbf{1} \cdot \mathrm{Pd}(\mathrm{allyl}) \mathrm{Cl}$. The anisotropic displacement factor exponent takes the form: $-2 \pi^{2}\left[\mathrm{~h}^{2} \mathrm{a} * 2 \mathrm{U}^{11}+\ldots+2 \mathrm{~h} \mathrm{k} \mathrm{a}^{*} \mathrm{~b}^{*} \mathrm{U}^{12}\right]$

\begin{tabular}{ccccccc}
\hline & $\mathrm{U}^{11}$ & $\mathrm{U}^{22}$ & $\mathrm{U}^{33}$ & $\mathrm{U}^{23}$ & $\mathrm{U}^{13}$ & $\mathrm{U}^{12}$ \\
\hline $\operatorname{Pd}(1)$ & $10(1)$ & $5(1)$ & $11(1)$ & $-3(1)$ & $-1(1)$ & $0(1)$ \\
$\operatorname{Pd}(2)$ & $11(1)$ & $5(1)$ & $12(1)$ & $-4(1)$ & $-1(1)$ & $-1(1)$
\end{tabular}




\begin{tabular}{|c|c|c|c|c|c|c|}
\hline $\mathrm{Cl}(1)$ & $14(1)$ & $15(1)$ & $16(1)$ & $-7(1)$ & $0(1)$ & 1(1) \\
\hline $\mathrm{Cl}(2)$ & $15(1)$ & $16(1)$ & $16(1)$ & $-10(1)$ & $-3(1)$ & $-1(1)$ \\
\hline $\mathrm{S}(1)$ & 11(1) & $4(1)$ & $10(1)$ & $-3(1)$ & $0(1)$ & $-2(1)$ \\
\hline $\mathrm{S}(2)$ & 11(1) & $5(1)$ & $14(1)$ & $-4(1)$ & $0(1)$ & $0(1)$ \\
\hline $\mathrm{P}(1)$ & $8(1)$ & $5(1)$ & $9(1)$ & $-3(1)$ & $0(1)$ & $-2(1)$ \\
\hline $\mathrm{P}(2)$ & $8(1)$ & $6(1)$ & $9(1)$ & $-4(1)$ & $-1(1)$ & 1(1) \\
\hline $\mathrm{P}(3)$ & $9(1)$ & $7(1)$ & $12(1)$ & $-5(1)$ & 1(1) & $-1(1)$ \\
\hline $\mathrm{P}(4)$ & $9(1)$ & $6(1)$ & $10(1)$ & $-4(1)$ & $0(1)$ & $-1(1)$ \\
\hline $\mathrm{O}(1)$ & $9(2)$ & $12(2)$ & $16(2)$ & $-7(1)$ & $3(1)$ & $-4(1)$ \\
\hline $\mathrm{O}(2)$ & $20(2)$ & $5(1)$ & $13(2)$ & $-2(1)$ & $-6(1)$ & $3(1)$ \\
\hline $\mathrm{O}(3)$ & $15(2)$ & $10(2)$ & $14(2)$ & $-5(1)$ & $5(1)$ & $-7(1)$ \\
\hline $\mathrm{O}(4)$ & $12(2)$ & $9(2)$ & $18(2)$ & $-6(1)$ & $-3(1)$ & $2(1)$ \\
\hline $\mathrm{C}(1)$ & $8(2)$ & $7(2)$ & $11(2)$ & $-5(1)$ & $0(1)$ & $-1(1)$ \\
\hline$C(2)$ & $12(2)$ & $3(2)$ & $11(2)$ & $-3(2)$ & $-1(2)$ & $1(2)$ \\
\hline $\mathrm{C}(3)$ & $11(2)$ & $8(2)$ & $16(2)$ & $-5(2)$ & $1(2)$ & $-2(2)$ \\
\hline$C(4)$ & $18(2)$ & $12(2)$ & $13(2)$ & $-8(2)$ & $-3(2)$ & $-5(2)$ \\
\hline $\mathrm{C}(5)$ & $21(2)$ & $7(2)$ & $12(2)$ & $-5(2)$ & $0(2)$ & $4(2)$ \\
\hline$C(6)$ & $11(2)$ & $10(2)$ & $16(2)$ & $-4(2)$ & $4(2)$ & $-1(2)$ \\
\hline$C(7)$ & $9(2)$ & $10(2)$ & $22(2)$ & $-9(2)$ & $-3(2)$ & $1(2)$ \\
\hline $\mathrm{C}(8)$ & $27(3)$ & $30(3)$ & $20(3)$ & $-16(2)$ & $5(2)$ & $-3(2)$ \\
\hline $\mathrm{C}(9)$ & $12(2)$ & $9(2)$ & $12(2)$ & $-6(2)$ & $1(2)$ & $-2(2)$ \\
\hline$C(10)$ & $22(3)$ & $13(2)$ & $12(2)$ & $-5(2)$ & $-1(2)$ & $-3(2)$ \\
\hline$C(11)$ & $30(3)$ & $18(3)$ & $16(2)$ & $-3(2)$ & $7(2)$ & $-13(2)$ \\
\hline$C(12)$ & $31(3)$ & $29(3)$ & $6(2)$ & $-6(2)$ & $4(2)$ & $-5(2)$ \\
\hline $\mathrm{C}(13)$ & $36(3)$ & $28(3)$ & $17(3)$ & $-13(2)$ & $-10(2)$ & $-3(2)$ \\
\hline$C(14)$ & $21(3)$ & $18(2)$ & $16(2)$ & $-5(2)$ & $-1(2)$ & $-8(2)$ \\
\hline$C(15)$ & $9(2)$ & $8(2)$ & $9(2)$ & $-2(2)$ & $-2(2)$ & $-3(2)$ \\
\hline$C(16)$ & $11(2)$ & $16(2)$ & $19(2)$ & $-11(2)$ & $3(2)$ & $-4(2)$ \\
\hline $\mathrm{C}(17)$ & $14(2)$ & $16(2)$ & $17(2)$ & $-10(2)$ & $1(2)$ & $0(2)$ \\
\hline $\mathrm{C}(18)$ & $19(2)$ & $10(2)$ & $25(3)$ & $-11(2)$ & $1(2)$ & $-4(2)$ \\
\hline$C(19)$ & $11(2)$ & $10(2)$ & $23(2)$ & $-6(2)$ & $3(2)$ & $-5(2)$ \\
\hline $\mathrm{C}(20)$ & $12(2)$ & $7(2)$ & $19(2)$ & $-7(2)$ & $-1(2)$ & $-1(2)$ \\
\hline $\mathrm{C}(21)$ & $8(2)$ & $8(2)$ & $13(2)$ & $-6(2)$ & $-2(2)$ & $-1(2)$ \\
\hline$C(22)$ & $7(2)$ & $9(2)$ & $20(2)$ & $-7(2)$ & $-1(2)$ & $-2(2)$ \\
\hline $\mathrm{C}(23)$ & $12(2)$ & $12(2)$ & $19(2)$ & $-8(2)$ & $0(2)$ & $-2(2)$ \\
\hline $\mathrm{C}(24)$ & $10(2)$ & $14(2)$ & $32(3)$ & $-10(2)$ & $-2(2)$ & $2(2)$ \\
\hline$C(25)$ & $15(2)$ & $13(2)$ & $26(3)$ & $-5(2)$ & $-10(2)$ & $4(2)$ \\
\hline$C(26)$ & $18(2)$ & $7(2)$ & $16(2)$ & $-1(2)$ & $-6(2)$ & $-4(2)$ \\
\hline $\mathrm{C}(27)$ & $11(2)$ & $8(2)$ & $14(2)$ & $-7(2)$ & $0(2)$ & $-4(2)$ \\
\hline $\mathrm{C}(28)$ & $13(2)$ & $18(2)$ & $15(2)$ & $-10(2)$ & $0(2)$ & $1(2)$ \\
\hline $\mathrm{C}(29)$ & $23(3)$ & $31(3)$ & $21(3)$ & $-19(2)$ & $6(2)$ & $-4(2)$ \\
\hline $\mathrm{C}(30)$ & $12(2)$ & $25(3)$ & $42(3)$ & $-22(3)$ & $7(2)$ & $-3(2)$ \\
\hline $\mathrm{C}(31)$ & $11(2)$ & $13(2)$ & $40(3)$ & $-13(2)$ & $0(2)$ & $2(2)$ \\
\hline$C(32)$ & $15(2)$ & $8(2)$ & $22(2)$ & $-6(2)$ & $0(2)$ & $-2(2)$ \\
\hline$C(33)$ & $10(2)$ & $3(2)$ & $12(2)$ & $-2(2)$ & $-4(2)$ & $4(2)$ \\
\hline$C(34)$ & $12(2)$ & $4(2)$ & $16(2)$ & $-3(2)$ & $-3(2)$ & $0(2)$ \\
\hline$C(35)$ & $25(3)$ & $14(2)$ & $14(2)$ & $-9(2)$ & $-3(2)$ & $2(2)$ \\
\hline$C(36)$ & $26(3)$ & $15(2)$ & $23(3)$ & $-14(2)$ & $-11(2)$ & $5(2)$ \\
\hline $\mathrm{C}(37)$ & $15(2)$ & $9(2)$ & $27(3)$ & $-10(2)$ & $-9(2)$ & $0(2)$ \\
\hline$C(38)$ & $14(2)$ & $7(2)$ & $16(2)$ & $-6(2)$ & $-4(2)$ & $1(2)$ \\
\hline C(39A) & $18(2)$ & $12(2)$ & $16(2)$ & $-6(2)$ & $-3(2)$ & $-3(2)$ \\
\hline $\mathrm{C}(40 \mathrm{~A})$ & $17(2)$ & $12(2)$ & $18(2)$ & $-6(2)$ & $-1(2)$ & $4(2)$ \\
\hline $\mathrm{C}(41 \mathrm{~A})$ & $17(2)$ & $10(2)$ & $17(2)$ & $-8(2)$ & $-1(2)$ & $3(2)$ \\
\hline$C(39 B)$ & $12(3)$ & $10(3)$ & $12(3)$ & $-4(2)$ & $-3(2)$ & $-1(2)$ \\
\hline $\mathrm{C}(40 \mathrm{~B})$ & $16(3)$ & $13(3)$ & $17(3)$ & $-7(2)$ & $-1(2)$ & $1(2)$ \\
\hline
\end{tabular}




\begin{tabular}{|c|c|c|c|c|c|c|}
\hline$C(41 B)$ & $15(3)$ & $13(3)$ & $14(3)$ & $-6(2)$ & $-2(2)$ & $2(2)$ \\
\hline $\mathrm{C}(42)$ & $8(2)$ & $5(2)$ & $11(2)$ & $-3(1)$ & 1(1) & $-2(1)$ \\
\hline $\mathrm{C}(43)$ & $11(2)$ & $6(2)$ & $17(2)$ & $-5(2)$ & $-1(2)$ & $-3(2)$ \\
\hline $\mathrm{C}(44)$ & $11(2)$ & $7(2)$ & $18(2)$ & $-4(2)$ & $3(2)$ & $-2(2)$ \\
\hline$C(45)$ & $10(2)$ & $9(2)$ & $19(2)$ & $-5(2)$ & $-5(2)$ & $-1(2)$ \\
\hline$C(46)$ & $18(2)$ & $8(2)$ & $16(2)$ & $-7(2)$ & $-1(2)$ & $-4(2)$ \\
\hline $\mathrm{C}(47)$ & $12(2)$ & $11(2)$ & $22(2)$ & $-9(2)$ & $1(2)$ & $3(2)$ \\
\hline $\mathrm{C}(48)$ & $10(2)$ & $7(2)$ & $17(2)$ & $-4(2)$ & $-1(2)$ & $-1(2)$ \\
\hline$C(49)$ & $25(3)$ & $23(3)$ & $24(3)$ & $-16(2)$ & $-7(2)$ & $5(2)$ \\
\hline$C(50)$ & $13(2)$ & $13(2)$ & $15(2)$ & $-5(2)$ & $3(2)$ & $-4(2)$ \\
\hline $\mathrm{C}(51)$ & $21(3)$ & $20(3)$ & $19(2)$ & $-9(2)$ & $1(2)$ & $-2(2)$ \\
\hline$C(52)$ & $32(3)$ & $34(3)$ & $20(3)$ & $-13(3)$ & $10(2)$ & $-6(2)$ \\
\hline$C(53)$ & $42(3)$ & $40(3)$ & $14(3)$ & $-11(3)$ & $0(2)$ & $-8(3)$ \\
\hline$C(54)$ & $34(3)$ & $16(3)$ & $17(3)$ & $-1(2)$ & $-8(2)$ & $-5(2)$ \\
\hline$C(55)$ & $20(2)$ & $9(2)$ & $19(2)$ & $-2(2)$ & $1(2)$ & $-6(2)$ \\
\hline$C(56)$ & $9(2)$ & $6(2)$ & $23(2)$ & $-8(2)$ & $2(2)$ & $1(2)$ \\
\hline$C(57)$ & $13(2)$ & $11(2)$ & $18(2)$ & $-8(2)$ & $1(2)$ & $0(2)$ \\
\hline $\mathrm{C}(58)$ & $13(2)$ & $8(2)$ & $30(3)$ & $-9(2)$ & $4(2)$ & $-1(2)$ \\
\hline $\mathrm{C}(59)$ & $10(2)$ & $18(2)$ & $34(3)$ & $-16(2)$ & $-5(2)$ & $0(2)$ \\
\hline$C(60)$ & $11(2)$ & $14(2)$ & $27(3)$ & $-11(2)$ & $-6(2)$ & $4(2)$ \\
\hline$C(61)$ & $9(2)$ & $8(2)$ & $19(2)$ & $-8(2)$ & $-1(2)$ & $1(2)$ \\
\hline$C(62)$ & $12(2)$ & $5(2)$ & $12(2)$ & $-5(2)$ & $1(2)$ & $1(2)$ \\
\hline$C(63)$ & $9(2)$ & $10(2)$ & $15(2)$ & $-6(2)$ & $1(2)$ & $-2(2)$ \\
\hline$C(64)$ & $15(2)$ & $12(2)$ & $18(2)$ & $-8(2)$ & $-1(2)$ & $3(2)$ \\
\hline$C(65)$ & $17(2)$ & $11(2)$ & $24(3)$ & $-12(2)$ & $3(2)$ & $2(2)$ \\
\hline $\mathrm{C}(66)$ & $18(2)$ & $18(2)$ & $27(3)$ & $-17(2)$ & $-2(2)$ & $-3(2)$ \\
\hline $\mathrm{C}(67)$ & $14(2)$ & $12(2)$ & $25(3)$ & $-11(2)$ & $-5(2)$ & $2(2)$ \\
\hline $\mathrm{C}(68)$ & $12(2)$ & $4(2)$ & $9(2)$ & $-3(2)$ & $5(2)$ & $-6(2)$ \\
\hline $\mathrm{C}(69)$ & $11(2)$ & $11(2)$ & $18(2)$ & $-7(2)$ & $0(2)$ & $-2(2)$ \\
\hline $\mathrm{C}(70)$ & $14(2)$ & $11(2)$ & $24(3)$ & $-8(2)$ & $4(2)$ & $-3(2)$ \\
\hline $\mathrm{C}(71)$ & $20(3)$ & $12(2)$ & $19(2)$ & $-9(2)$ & $9(2)$ & $-4(2)$ \\
\hline $\mathrm{C}(72)$ & $26(3)$ & $13(2)$ & $11(2)$ & $-6(2)$ & $3(2)$ & $-7(2)$ \\
\hline $\mathrm{C}(73)$ & $17(2)$ & $8(2)$ & $11(2)$ & $-4(2)$ & $1(2)$ & $-1(2)$ \\
\hline $\mathrm{C}(74)$ & $9(2)$ & $5(2)$ & $13(2)$ & $-6(2)$ & $0(2)$ & $3(2)$ \\
\hline $\mathrm{C}(75)$ & $11(2)$ & $9(2)$ & $16(2)$ & $-5(2)$ & $-4(2)$ & $1(2)$ \\
\hline $\mathrm{C}(76)$ & $10(2)$ & $12(2)$ & $20(2)$ & $-9(2)$ & $1(2)$ & $-3(2)$ \\
\hline $\mathrm{C}(77)$ & $15(2)$ & $22(3)$ & $26(3)$ & $-16(2)$ & $-4(2)$ & $1(2)$ \\
\hline $\mathrm{C}(78)$ & $20(3)$ & $20(3)$ & $12(2)$ & $-7(2)$ & $-5(2)$ & $0(2)$ \\
\hline $\mathrm{C}(79)$ & $15(2)$ & $11(2)$ & $11(2)$ & $-1(2)$ & $2(2)$ & $-1(2)$ \\
\hline $\mathrm{C}(80 \mathrm{~A})$ & $18(2)$ & $12(2)$ & $20(2)$ & $-9(2)$ & $-2(2)$ & $-3(2)$ \\
\hline $\mathrm{C}(81 \mathrm{~A})$ & $19(2)$ & $14(2)$ & $20(2)$ & $-7(2)$ & $0(2)$ & $-3(2)$ \\
\hline $\mathrm{C}(82 \mathrm{~A})$ & $20(2)$ & $12(2)$ & $20(2)$ & $-7(2)$ & $0(2)$ & $3(2)$ \\
\hline $\mathrm{C}(80 \mathrm{~B})$ & $13(3)$ & $12(3)$ & $13(4)$ & $-7(2)$ & $-1(2)$ & $-1(2)$ \\
\hline $\mathrm{C}(81 \mathrm{~B})$ & $16(3)$ & $13(3)$ & $15(3)$ & $-7(2)$ & $-2(2)$ & $0(2)$ \\
\hline $\mathrm{C}(82 \mathrm{~B})$ & 11(3) & $9(3)$ & $11(3)$ & $-4(2)$ & $1(2)$ & $1(2)$ \\
\hline $\mathrm{C} 111$ & $26(1)$ & $33(1)$ & 21(1) & $-7(1)$ & $3(1)$ & 1(1) \\
\hline $\mathrm{Cl} 21$ & $24(1)$ & $33(1)$ & $34(1)$ & $-20(1)$ & $2(1)$ & $-3(1)$ \\
\hline $\mathrm{C} 11$ & $33(4)$ & $23(3)$ & $37(4)$ & $-12(3)$ & $12(3)$ & $-14(3)$ \\
\hline $\mathrm{Cl12}$ & $23(1)$ & $31(1)$ & $26(1)$ & $-5(1)$ & $-2(1)$ & $-4(1)$ \\
\hline $\mathrm{Cl} 22$ & $45(1)$ & $29(1)$ & $38(1)$ & $-3(1)$ & $-6(1)$ & $-10(1)$ \\
\hline $\mathrm{C} 12$ & $34(4)$ & $25(3)$ & $36(4)$ & $-3(3)$ & $13(3)$ & $-2(3)$ \\
\hline
\end{tabular}




\subsection{Crystal Structure Determination of $1 \cdot \mathrm{Rh}(\mathrm{CO}) \mathrm{Cl}$}

Both solvent molecules $\left(\mathrm{CHCl}_{3}\right.$ and benzene) were disordered over two positions. They were modelled using appropriate PART, SAME, SIMU and RIGU instructions. The final occupancy was $0.67: 0.33$ for the $\mathrm{CHCl}_{3}$ and $0.53: 0.47$ for the benzene.

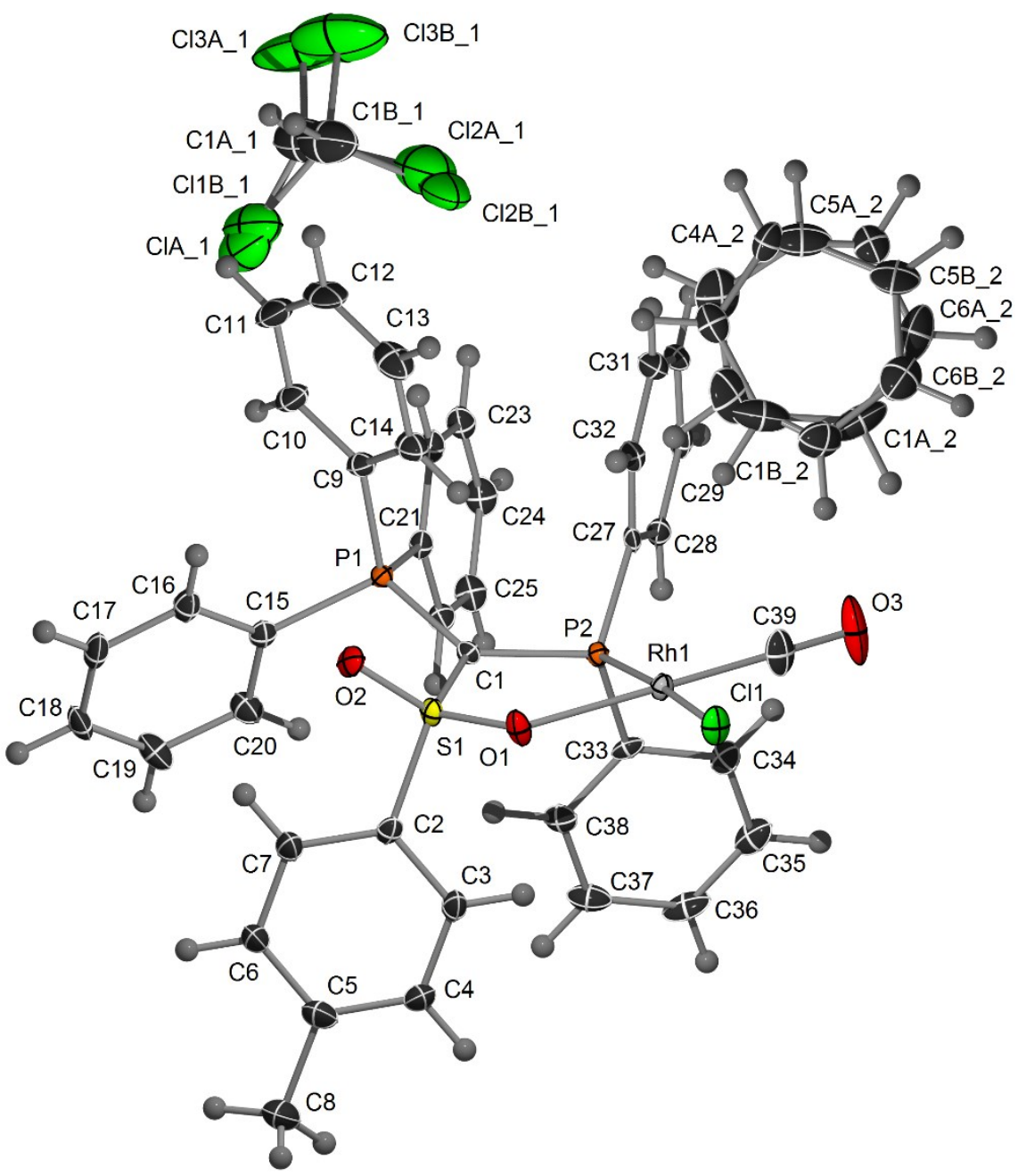

Figure S22. ORTEP Plot of $\mathbf{1} \cdot \mathrm{Rh}(\mathrm{CO}) \mathrm{Cl}$. Ellipsoids are drawn at the $50 \%$ probability level.

Table S11. Atomic coordinates ( $\left.\mathrm{x} 10^{4}\right)$ and equivalent isotropic displacement parameters $\left(\AA^{2} \times 10^{3}\right)$ for $1 \cdot \mathrm{Rh}(\mathrm{CO}) \mathrm{Cl}$. $\mathrm{U}(\mathrm{eq})$ is defined as one third of the trace of the orthogonalized $\mathrm{U}^{\mathrm{ij}}$ tensor.

\begin{tabular}{ccccc}
\hline & $\mathrm{x}$ & $\mathrm{y}$ & $\mathrm{z}$ & $\mathrm{U}(\mathrm{eq})$ \\
\hline $\mathrm{Rh}(1)$ & $9014(1)$ & $1360(1)$ & $6147(1)$ & $11(1)$ \\
$\mathrm{Cl}(1)$ & $9226(1)$ & $595(1)$ & $7759(1)$ & $19(1)$ \\
$\mathrm{S}(1)$ & $6252(1)$ & $1026(1)$ & $4050(1)$ & $12(1)$ \\
$\mathrm{P}(1)$ & $5722(1)$ & $2462(1)$ & $2770(1)$ & $11(1)$ \\
$\mathrm{P}(2)$ & $8627(1)$ & $2017(1)$ & $4560(1)$ & $10(1)$ \\
$\mathrm{O}(1)$ & $7183(2)$ & $789(1)$ & $5209(2)$ & $16(1)$ \\
$\mathrm{O}(2)$ & $4875(2)$ & $1150(1)$ & $3888(2)$ & $16(1)$ \\
$\mathrm{O}(3)$ & $11550(3)$ & $2202(2)$ & $7262(2)$ & $44(1)$
\end{tabular}




\begin{tabular}{|c|c|c|c|c|}
\hline $\mathrm{C}(1)$ & 6896(3) & $1831(2)$ & $3682(3)$ & $11(1)$ \\
\hline $\mathrm{C}(2)$ & $6247(3)$ & $249(2)$ & $3159(3)$ & $13(1)$ \\
\hline $\mathrm{C}(3)$ & 7407(3) & $-161(2)$ & $3345(3)$ & $18(1)$ \\
\hline $\mathrm{C}(4)$ & 7386(3) & $-756(2)$ & $2617(3)$ & $22(1)$ \\
\hline $\mathrm{C}(5)$ & 6213(4) & $-944(2)$ & 1693(3) & $24(1)$ \\
\hline $\mathrm{C}(6)$ & 5053(4) & $-534(2)$ & $1524(3)$ & $24(1)$ \\
\hline$C(7)$ & $5060(3)$ & $62(2)$ & $2244(3)$ & $20(1)$ \\
\hline $\mathrm{C}(8)$ & 6193(4) & $-1587(2)$ & $877(4)$ & $37(1)$ \\
\hline $\mathrm{C}(9)$ & 4806(3) & 2999(2) & $3449(3)$ & $16(1)$ \\
\hline$C(10)$ & 3808(3) & $3511(2)$ & 2803(3) & $22(1)$ \\
\hline $\mathrm{C}(11)$ & 3084(4) & $3916(2)$ & $3306(4)$ & $32(1)$ \\
\hline$C(12)$ & 3348(4) & $3804(2)$ & 4447(4) & $35(1)$ \\
\hline C(13) & 4324(4) & $3293(2)$ & $5090(3)$ & $29(1)$ \\
\hline$C(14)$ & $5054(3)$ & $2884(2)$ & 4591(3) & $20(1)$ \\
\hline$C(15)$ & 4455(3) & 1973(2) & 1572(3) & $14(1)$ \\
\hline$C(16)$ & 3148(3) & $1888(2)$ & 1523(3) & $18(1)$ \\
\hline$C(17)$ & 2190(3) & $1508(2)$ & 602(3) & $22(1)$ \\
\hline $\mathrm{C}(18)$ & 2503(3) & $1217(2)$ & $-267(3)$ & $21(1)$ \\
\hline$C(19)$ & 3794(3) & $1298(2)$ & $-221(3)$ & $23(1)$ \\
\hline$C(20)$ & 4771(4) & $1666(2)$ & 698(3) & $20(1)$ \\
\hline$C(21)$ & $6570(3)$ & $3138(2)$ & $2205(3)$ & $13(1)$ \\
\hline$C(22)$ & 6453(3) & $3929(2)$ & $2335(3)$ & $16(1)$ \\
\hline$C(23)$ & 7104(3) & $4442(2)$ & 1890(3) & $19(1)$ \\
\hline$C(24)$ & 7870(3) & $4174(2)$ & 1318(3) & $20(1)$ \\
\hline$C(25)$ & 7988(3) & $3392(2)$ & 1181(3) & $19(1)$ \\
\hline$C(26)$ & 7348(3) & $2874(2)$ & 1631(3) & $15(1)$ \\
\hline $\mathrm{C}(27)$ & $8870(3)$ & $3056(2)$ & $4696(2)$ & $12(1)$ \\
\hline $\mathrm{C}(28)$ & 9554(3) & $3463(2)$ & $4148(3)$ & $15(1)$ \\
\hline$C(29)$ & $9660(3)$ & $4265(2)$ & $4261(3)$ & $18(1)$ \\
\hline$C(30)$ & 9090(3) & $4643(2)$ & 4919(3) & $20(1)$ \\
\hline $\mathrm{C}(31)$ & 8445(3) & $4234(2)$ & $5478(3)$ & $21(1)$ \\
\hline $\mathrm{C}(32)$ & 8349(3) & $3441(2)$ & $5380(3)$ & $17(1)$ \\
\hline $\mathrm{C}(33)$ & 9686(3) & $1689(2)$ & $3835(3)$ & $13(1)$ \\
\hline$C(34)$ & 11074(3) & $1817(2)$ & $4364(3)$ & $17(1)$ \\
\hline$C(35)$ & 11928(3) & $1485(2)$ & $3926(3)$ & $23(1)$ \\
\hline$C(36)$ & $11426(4)$ & $1015(2)$ & 2982(3) & $24(1)$ \\
\hline$C(37)$ & 10053(4) & $886(2)$ & 2443(3) & $22(1)$ \\
\hline$C(38)$ & 9191(3) & $1226(2)$ & 2872(3) & $16(1)$ \\
\hline$C(39)$ & $10559(4)$ & $1868(2)$ & 6841(3) & $24(1)$ \\
\hline Cl1A1 & $3558(11)$ & $5310(4)$ & $970(8)$ & $55(1)$ \\
\hline $\mathrm{Cl} 2 \mathrm{~A} 1$ & $5306(8)$ & $5796(4)$ & $3207(6)$ & $70(1)$ \\
\hline $\mathrm{Cl3A} 1$ & $3780(6)$ & $6928(2)$ & $1576(8)$ & $100(2)$ \\
\hline C1A1 & $3769(11)$ & $5986(5)$ & 2071(9) & $54(2)$ \\
\hline Cl1B1 & $3570(20)$ & $5453(10)$ & $1113(18)$ & $55(2)$ \\
\hline $\mathrm{Cl} 2 \mathrm{~B} 1$ & $5517(13)$ & $5596(7)$ & $3501(11)$ & $63(2)$ \\
\hline $\mathrm{Cl3B} 1$ & $4014(12)$ & $6891(5)$ & $2273(15)$ & $108(2)$ \\
\hline C1B1 & $3970(20)$ & $5902(10)$ & $2481(16)$ & $56(2)$ \\
\hline C1A2 & $10262(15)$ & $3154(9)$ & $9097(12)$ & $42(3)$ \\
\hline $\mathrm{C} 2 \mathrm{~A} 2$ & $8973(18)$ & $3069(8)$ & $8383(16)$ & $40(4)$ \\
\hline C3A2 & $8046(14)$ & $3686(8)$ & $8202(14)$ & $22(3)$ \\
\hline C4A2 & $8486(13)$ & $4341(9)$ & $8805(15)$ & $23(3)$ \\
\hline $\mathrm{C} 5 \mathrm{~A} 2$ & $9820(13)$ & $4432(7)$ & $9615(10)$ & $23(2)$ \\
\hline C6A2 & $10699(10)$ & $3845(10)$ & $9749(8)$ & $39(3)$ \\
\hline C1B2 & $9736(17)$ & $2896(7)$ & $8931(11)$ & $30(3)$ \\
\hline C2B2 & $8523(18)$ & $3184(11)$ & $8174(16)$ & $40(4)$ \\
\hline
\end{tabular}




\begin{tabular}{ccccc} 
C3B2 & $8050(20)$ & $3940(11)$ & $8205(19)$ & $36(4)$ \\
C4B2 & $8920(20)$ & $4403(10)$ & $9051(18)$ & $36(4)$ \\
C5B2 & $10184(17)$ & $4174(9)$ & $9840(13)$ & $33(3)$ \\
C6B2 & $10532(11)$ & $3393(11)$ & $9787(10)$ & $37(3)$ \\
\hline
\end{tabular}

Table S12. Anisotropic displacement parameters $\left(\AA^{2} \times 10^{3}\right)$ for $\mathbf{1} \cdot \mathrm{Rh}(\mathrm{CO}) \mathrm{Cl}$. The anisotropic displacement factor exponent takes the form: $-2 \pi^{2}\left[h^{2} a * 2 U^{11}+\ldots+2 h k a^{*} b^{*} U^{12}\right]$

\begin{tabular}{|c|c|c|c|c|c|c|}
\hline & $\mathrm{U}^{11}$ & $\mathrm{U}^{22}$ & $\mathrm{U}^{33}$ & $\mathrm{U}^{23}$ & $\mathrm{U}^{13}$ & $\mathrm{U}^{12}$ \\
\hline $\mathrm{Rh}(1)$ & $10(1)$ & 11(1) & $12(1)$ & $1(1)$ & $3(1)$ & $-1(1)$ \\
\hline $\mathrm{Cl}(1)$ & $17(1)$ & $22(1)$ & $15(1)$ & $6(1)$ & $4(1)$ & $-3(1)$ \\
\hline $\mathrm{S}(1)$ & $10(1)$ & $12(1)$ & $13(1)$ & 1(1) & $4(1)$ & $-2(1)$ \\
\hline $\mathrm{P}(1)$ & $9(1)$ & $10(1)$ & $12(1)$ & 1(1) & $4(1)$ & $0(1)$ \\
\hline $\mathrm{P}(2)$ & $8(1)$ & $10(1)$ & $12(1)$ & $0(1)$ & $4(1)$ & $-1(1)$ \\
\hline $\mathrm{O}(1)$ & $16(1)$ & $16(1)$ & $13(1)$ & $3(1)$ & 1(1) & $-6(1)$ \\
\hline $\mathrm{O}(2)$ & $11(1)$ & $16(1)$ & $21(1)$ & $0(1)$ & $8(1)$ & $-2(1)$ \\
\hline $\mathrm{O}(3)$ & $29(2)$ & $62(2)$ & $26(2)$ & $9(1)$ & $-5(1)$ & $-29(2)$ \\
\hline $\mathrm{C}(1)$ & $10(1)$ & $8(1)$ & $13(1)$ & $0(1)$ & $5(1)$ & $-2(1)$ \\
\hline $\mathrm{C}(2)$ & $12(2)$ & $10(1)$ & $17(2)$ & $4(1)$ & $4(1)$ & $0(1)$ \\
\hline C(3) & $11(2)$ & $15(2)$ & $23(2)$ & $0(1)$ & $2(1)$ & $-1(1)$ \\
\hline $\mathrm{C}(4)$ & $17(2)$ & $16(2)$ & $28(2)$ & $-2(2)$ & $6(2)$ & $4(1)$ \\
\hline$C(5)$ & $28(2)$ & $15(2)$ & $26(2)$ & $-5(2)$ & $8(2)$ & $-1(2)$ \\
\hline$C(6)$ & $21(2)$ & $16(2)$ & $25(2)$ & $-5(1)$ & $-2(1)$ & 1(1) \\
\hline $\mathrm{C}(7)$ & $15(2)$ & $14(2)$ & $24(2)$ & $-1(1)$ & 1(1) & $-1(1)$ \\
\hline $\mathrm{C}(8)$ & $37(2)$ & $27(2)$ & $36(2)$ & $-14(2)$ & $2(2)$ & $7(2)$ \\
\hline $\mathrm{C}(9)$ & $13(2)$ & $12(1)$ & $24(2)$ & $-2(1)$ & $10(1)$ & $-1(1)$ \\
\hline $\mathrm{C}(10)$ & $15(2)$ & $16(2)$ & $35(2)$ & $0(2)$ & $11(2)$ & $2(1)$ \\
\hline $\mathrm{C}(11)$ & $19(2)$ & $20(2)$ & $54(3)$ & $-3(2)$ & $13(2)$ & $6(2)$ \\
\hline $\mathrm{C}(12)$ & $26(2)$ & $30(2)$ & $56(3)$ & $-20(2)$ & $25(2)$ & $0(2)$ \\
\hline$C(13)$ & $29(2)$ & $36(2)$ & $28(2)$ & $-12(2)$ & $18(2)$ & $-7(2)$ \\
\hline$C(14)$ & $18(2)$ & $20(2)$ & $23(2)$ & $-6(1)$ & $10(1)$ & $-2(1)$ \\
\hline$C(15)$ & $14(2)$ & 11(1) & $14(1)$ & $3(1)$ & $4(1)$ & $1(1)$ \\
\hline$C(16)$ & $13(2)$ & $16(2)$ & $21(2)$ & $2(1)$ & $4(1)$ & $-1(1)$ \\
\hline$C(17)$ & $12(2)$ & $18(2)$ & $28(2)$ & $5(1)$ & $0(1)$ & $-2(1)$ \\
\hline$C(18)$ & $21(2)$ & $14(2)$ & $18(2)$ & $-1(1)$ & $-3(1)$ & $-6(1)$ \\
\hline$C(19)$ & $28(2)$ & $23(2)$ & $18(1)$ & $-6(2)$ & $8(1)$ & $-8(2)$ \\
\hline$C(20)$ & $20(2)$ & $21(2)$ & $21(2)$ & $-4(1)$ & $9(1)$ & $-4(1)$ \\
\hline$C(21)$ & $10(2)$ & $13(1)$ & $13(1)$ & $2(1)$ & $3(1)$ & $-1(1)$ \\
\hline$C(22)$ & $13(2)$ & $18(2)$ & $16(2)$ & $2(1)$ & $4(1)$ & $-1(1)$ \\
\hline$C(23)$ & $20(2)$ & $14(2)$ & $18(2)$ & $3(1)$ & $2(1)$ & $-2(1)$ \\
\hline$C(24)$ & $21(2)$ & $21(2)$ & $18(2)$ & $5(1)$ & $7(1)$ & $-5(2)$ \\
\hline$C(25)$ & $19(2)$ & $23(2)$ & $16(2)$ & $1(1)$ & $9(1)$ & $-3(1)$ \\
\hline $\mathrm{C}(26)$ & $15(2)$ & $14(2)$ & $15(2)$ & $-1(1)$ & $4(1)$ & $0(1)$ \\
\hline $\mathrm{C}(27)$ & $8(1)$ & $10(1)$ & $12(1)$ & $-1(1)$ & $-1(1)$ & $-3(1)$ \\
\hline $\mathrm{C}(28)$ & $11(2)$ & $13(2)$ & $16(2)$ & 1(1) & $2(1)$ & $0(1)$ \\
\hline $\mathrm{C}(29)$ & $11(2)$ & $14(2)$ & $23(2)$ & $5(1)$ & 1(1) & $-4(1)$ \\
\hline $\mathrm{C}(30)$ & $16(2)$ & $11(2)$ & $25(2)$ & $-4(1)$ & $-1(1)$ & $-1(1)$ \\
\hline $\mathrm{C}(31)$ & $19(2)$ & $20(2)$ & $24(2)$ & $-8(1)$ & $7(1)$ & $-1(1)$ \\
\hline $\mathrm{C}(32)$ & $14(2)$ & $16(2)$ & $20(2)$ & $-3(1)$ & $6(1)$ & $-3(1)$ \\
\hline $\mathrm{C}(33)$ & $15(2)$ & $9(1)$ & $19(2)$ & $4(1)$ & $10(1)$ & $4(1)$ \\
\hline $\mathrm{C}(34)$ & $14(2)$ & $19(2)$ & $21(2)$ & 2(1) & $10(1)$ & $-1(1)$ \\
\hline$C(35)$ & $17(2)$ & $21(2)$ & $38(2)$ & $7(2)$ & $16(1)$ & $0(1)$ \\
\hline $\mathrm{C}(36)$ & $28(2)$ & $20(2)$ & $38(2)$ & $7(2)$ & $28(2)$ & $7(2)$ \\
\hline
\end{tabular}




\begin{tabular}{lcccccc}
$\mathrm{C}(37)$ & $33(2)$ & $16(2)$ & $26(2)$ & $-2(1)$ & $21(2)$ & $-1(2)$ \\
$\mathrm{C}(38)$ & $18(2)$ & $12(2)$ & $20(1)$ & $1(1)$ & $10(1)$ & $-2(1)$ \\
$\mathrm{C}(39)$ & $21(2)$ & $29(2)$ & $17(2)$ & $8(2)$ & $2(1)$ & $-6(2)$ \\
$\mathrm{C} 11 \mathrm{~A} 1$ & $41(1)$ & $38(3)$ & $89(3)$ & $7(2)$ & $28(2)$ & $1(2)$ \\
$\mathrm{C} 12 \mathrm{~A} 1$ & $72(3)$ & $57(2)$ & $86(3)$ & $-20(2)$ & $38(2)$ & $-5(2)$ \\
$\mathrm{Cl} 1 \mathrm{~A} 1$ & $142(3)$ & $32(1)$ & $182(5)$ & $24(2)$ & $123(4)$ & $24(2)$ \\
$\mathrm{C} 1 \mathrm{~A} 1$ & $56(3)$ & $37(3)$ & $87(4)$ & $-8(3)$ & $46(3)$ & $-7(3)$ \\
$\mathrm{C} 11 \mathrm{~B} 1$ & $49(3)$ & $41(4)$ & $89(4)$ & $-3(3)$ & $41(3)$ & $-3(3)$ \\
$\mathrm{C} 12 \mathrm{~B} 1$ & $47(3)$ & $63(5)$ & $83(5)$ & $-46(4)$ & $30(3)$ & $-6(3)$ \\
$\mathrm{C} 13 \mathrm{~B} 1$ & $142(4)$ & $30(2)$ & $191(7)$ & $4(4)$ & $109(6)$ & $-2(3)$ \\
$\mathrm{C} 1 \mathrm{~B} 1$ & $57(3)$ & $38(3)$ & $87(5)$ & $-11(4)$ & $45(4)$ & $-6(3)$ \\
$\mathrm{C} 1 \mathrm{~A} 2$ & $45(7)$ & $39(7)$ & $50(7)$ & $16(6)$ & $28(6)$ & $26(6)$ \\
$\mathrm{C} 2 \mathrm{~A} 2$ & $65(10)$ & $22(5)$ & $42(10)$ & $-5(6)$ & $31(7)$ & $3(6)$ \\
$\mathrm{C} 3 \mathrm{~A} 2$ & $23(5)$ & $26(7)$ & $18(5)$ & $-1(5)$ & $9(4)$ & $-7(5)$ \\
$\mathrm{C} 4 \mathrm{~A} 2$ & $18(5)$ & $27(5)$ & $23(5)$ & $7(4)$ & $5(4)$ & $4(4)$ \\
$\mathrm{C} 5 \mathrm{~A} 2$ & $24(6)$ & $30(5)$ & $21(5)$ & $-2(4)$ & $13(4)$ & $-4(4)$ \\
$\mathrm{C} 6 \mathrm{~A} 2$ & $25(5)$ & $58(9)$ & $31(5)$ & $15(5)$ & $7(4)$ & $4(5)$ \\
$\mathrm{C} 1 \mathrm{~B} 2$ & $39(8)$ & $23(5)$ & $28(6)$ & $7(4)$ & $12(5)$ & $6(5)$ \\
$\mathrm{C} 2 \mathrm{~B} 2$ & $52(9)$ & $45(9)$ & $16(5)$ & $9(6)$ & $6(6)$ & $-7(6)$ \\
$\mathrm{C} 3 \mathrm{~B} 2$ & $42(7)$ & $41(10)$ & $23(7)$ & $15(7)$ & $11(5)$ & $-1(7)$ \\
$\mathrm{C} 4 \mathrm{~B} 2$ & $59(11)$ & $24(6)$ & $40(11)$ & $3(6)$ & $36(8)$ & $6(7)$ \\
$\mathrm{C} 5 \mathrm{~B} 2$ & $43(8)$ & $36(8)$ & $31(7)$ & $-9(6)$ & $26(6)$ & $4(6)$ \\
$\mathrm{C} 6 \mathrm{~B} 2$ & $33(6)$ & $46(8)$ & $32(6)$ & $3(6)$ & $13(4)$ & $7(5)$ \\
\hline
\end{tabular}




\subsection{Crystal Structure Determination of 5}

There are two $\mathrm{CHCl}_{3}$ solvents in the asymmetric unit. One of them lies on an inversion center and was modelled using PART -1 with an occupancy of 0.5 and no further restraints. The second $\mathrm{CHCl}_{3}$ was disordered over two positions and was modelled using appropriate PART, SAME, SIMU and RIGU instructions. The final occupancy was 0.74:0.26.

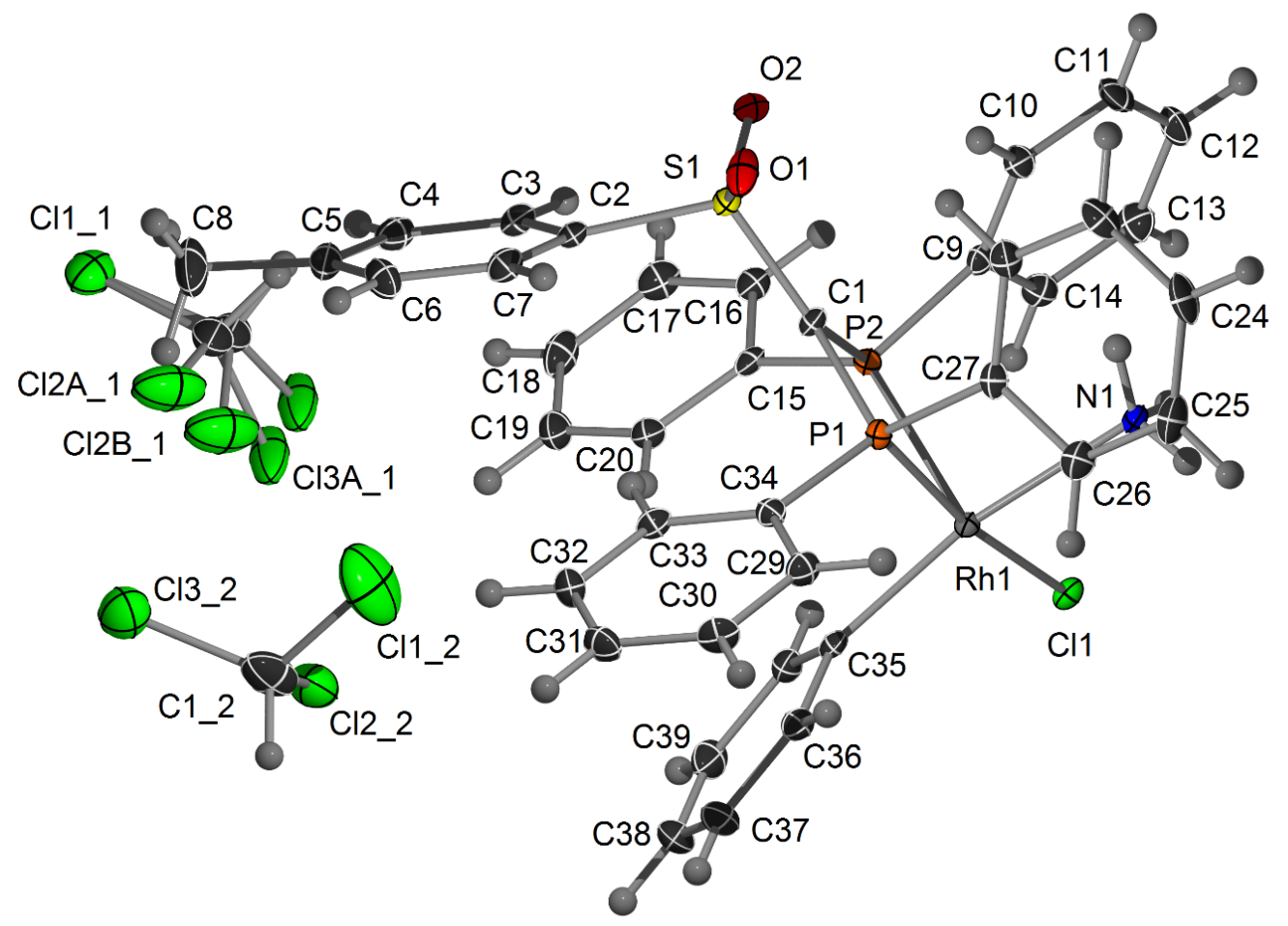

Figure S23. ORTEP Plot of 5. Ellipsoids are drawn at the 50\% probability level.

Table S13. Atomic coordinates $\left(\mathrm{x} 10^{4}\right)$ and equivalent isotropic displacement parameters $\left(\AA^{2} \times 10^{3}\right)$ for $\mathbf{5}$. $U(\mathrm{eq})$ is defined as one third of the trace of the orthogonalized $\mathrm{Uij}^{\mathrm{ij}}$ tensor.

\begin{tabular}{lllll}
\hline & $\mathrm{x}$ & \multicolumn{1}{c}{$\mathrm{y}$} & $\mathrm{Z}$ & $\mathrm{U}(\mathrm{eq})$ \\
\hline $\mathrm{Rh}(1)$ & $5776(1)$ & $-193(1)$ & $9016(1)$ & $8(1)$ \\
$\mathrm{Cl}(1)$ & $6523(1)$ & $-45(1)$ & $10321(1)$ & $11(1)$ \\
$\mathrm{S}(1)$ & $7736(1)$ & $-1092(1)$ & $6639(1)$ & $12(1)$ \\
$\mathrm{P}(1)$ & $7821(1)$ & $-599(1)$ & $8341(1)$ & $9(1)$ \\
$\mathrm{P}(2)$ & $5338(1)$ & $-508(1)$ & $7836(1)$ & $9(1)$ \\
$\mathrm{O}(1)$ & $9263(2)$ & $-1672(1)$ & $6770(1)$ & $21(1)$ \\
$\mathrm{O}(2)$ & $6933(2)$ & $-1687(1)$ & $6429(1)$ & $20(1)$ \\
$\mathrm{N}(1)$ & $6319(2)$ & $-2043(1)$ & $9764(1)$ & $11(1)$ \\
$\mathrm{C}(1)$ & $7066(2)$ & $-747(2)$ & $7507(1)$ & $10(1)$ \\
$\mathrm{C}(2)$ & $7439(2)$ & $166(2)$ & $5761(1)$ & $12(1)$ \\
$\mathrm{C}(3)$ & $6168(2)$ & $670(2)$ & $5274(1)$ & $15(1)$ \\
$\mathrm{C}(4)$ & $6012(2)$ & $1575(2)$ & $4538(1)$ & $18(1)$ \\
$\mathrm{C}(5)$ & $7105(3)$ & $1981(2)$ & $4283(1)$ & $19(1)$ \\
$\mathrm{C}(6)$ & $8349(2)$ & $1482(2)$ & $4796(2)$ & $19(1)$ \\
$\mathrm{C}(7)$ & $8525(2)$ & $580(2)$ & $5528(1)$ & $15(1)$
\end{tabular}




\begin{tabular}{|c|c|c|c|c|}
\hline$C(8)$ & $6955(3)$ & $2909(2)$ & $3452(2)$ & $34(1)$ \\
\hline$C(9)$ & $4898(2)$ & $-1789(2)$ & $8174(1)$ & $11(1)$ \\
\hline$C(10)$ & $5885(2)$ & $-2858(2)$ & $8186(1)$ & $14(1)$ \\
\hline $\mathrm{C}(11)$ & $5576(2)$ & $-3845(2)$ & $8552(1)$ & $18(1)$ \\
\hline$C(12)$ & $4305(3)$ & $-3777(2)$ & $8913(2)$ & $20(1)$ \\
\hline$C(13)$ & $3300(3)$ & $-2709(2)$ & $8896(2)$ & $20(1)$ \\
\hline$C(14)$ & $3604(2)$ & $-1724(2)$ & $8536(1)$ & $14(1)$ \\
\hline$C(15)$ & $4015(2)$ & $503(2)$ & 6993(1) & $12(1)$ \\
\hline$C(16)$ & $3044(2)$ & $178(2)$ & $6689(1)$ & $15(1)$ \\
\hline $\mathrm{C}(17)$ & $2107(2)$ & $941(2)$ & 6011(1) & 19(1) \\
\hline $\mathrm{C}(18)$ & $2109(3)$ & 2034(2) & $5640(1)$ & $22(1)$ \\
\hline$C(19)$ & $3075(3)$ & $2367(2)$ & 5929(1) & $20(1)$ \\
\hline$C(20)$ & $4039(2)$ & $1605(2)$ & $6595(1)$ & $14(1)$ \\
\hline$C(22)$ & $9723(2)$ & $-2901(2)$ & $8726(1)$ & $15(1)$ \\
\hline $\mathrm{C}(23)$ & $10644(2)$ & $-3940(2)$ & $9261(2)$ & $18(1)$ \\
\hline$C(24)$ & $10996(2)$ & $-4036(2)$ & $10066(2)$ & 19(1) \\
\hline$C(25)$ & $10439(2)$ & $-3084(2)$ & $10336(1)$ & 19(1) \\
\hline $\mathrm{C}(26)$ & $9524(2)$ & $-2039(2)$ & $9805(1)$ & $15(1)$ \\
\hline $\mathrm{C}(27)$ & $9154(2)$ & $-1941(2)$ & 8993(1) & $12(1)$ \\
\hline $\mathrm{C}(29)$ & $9839(2)$ & $352(2)$ & $8462(1)$ & $14(1)$ \\
\hline $\mathrm{C}(30)$ & $10414(2)$ & $1202(2)$ & $8197(1)$ & $16(1)$ \\
\hline $\mathrm{C}(31)$ & $9904(2)$ & 2099(2) & $7463(2)$ & $18(1)$ \\
\hline$C(32)$ & $8789(2)$ & $2166(2)$ & $6987(1)$ & $17(1)$ \\
\hline$C(33)$ & $8195(2)$ & $1334(2)$ & $7253(1)$ & $13(1)$ \\
\hline $\mathrm{C}(34)$ & $8716(2)$ & $415(2)$ & 7991(1) & $11(1)$ \\
\hline$C(35)$ & $5163(2)$ & $1546(2)$ & $8514(1)$ & $12(1)$ \\
\hline$C(36)$ & $6037(2)$ & 2112(2) & $8619(1)$ & $16(1)$ \\
\hline $\mathrm{C}(37)$ & $5538(3)$ & $3307(2)$ & $8399(2)$ & $22(1)$ \\
\hline $\mathrm{C}(38)$ & $4159(3)$ & $3975(2)$ & $8068(2)$ & $26(1)$ \\
\hline C(39) & $3281(3)$ & $3438(2)$ & $7959(2)$ & $21(1)$ \\
\hline $\mathrm{C}(40)$ & $3775(2)$ & $2243(2)$ & $8183(1)$ & $16(1)$ \\
\hline Cl11 & $719(1)$ & $4800(1)$ & $2332(1)$ & $29(1)$ \\
\hline $\mathrm{Cl} 2 \mathrm{~A} 1$ & $2987(2)$ & $4603(1)$ & $3367(1)$ & $46(1)$ \\
\hline $\mathrm{Cl3A} 1$ & 203(3) & $4920(1)$ & $3996(1)$ & $55(1)$ \\
\hline C1A1 & 1449(9) & $4295(8)$ & $3361(6)$ & $30(2)$ \\
\hline $\mathrm{Cl} 2 \mathrm{~B} 1$ & $2282(8)$ & $4648(4)$ & 3751(4) & $66(2)$ \\
\hline $\mathrm{Cl3B} 1$ & $-524(6)$ & $4578(5)$ & $3936(2)$ & $57(2)$ \\
\hline C1B1 & $1110(30)$ & $4190(30)$ & $3420(18)$ & $30(3)$ \\
\hline $\mathrm{Cl} 12$ & $4305(2)$ & $5972(2)$ & $4521(2)$ & $58(1)$ \\
\hline $\mathrm{Cl} 22$ & $7226(2)$ & 4401(1) & $4784(1)$ & $33(1)$ \\
\hline $\mathrm{Cl} 32$ & $5195(2)$ & 4046(1) & $6032(1)$ & $36(1)$ \\
\hline C12 & $5449(7)$ & $4540(5)$ & $4950(4)$ & $36(1)$ \\
\hline
\end{tabular}

Table S14. Anisotropic displacement parameters $\left(\AA^{2} \times 10^{3}\right)$ for 5 . The anisotropic displacement factor exponent takes the form: $-2 \pi^{2}\left[h^{2} a^{* 2} U^{11}+\ldots+2 h k a^{*} b^{*} U^{12}\right]$

\begin{tabular}{lllllll}
\hline & \multicolumn{1}{c}{$\mathrm{U}^{11}$} & \multicolumn{1}{c}{$\mathrm{U}^{22}$} & \multicolumn{1}{c}{$\mathrm{U}^{33}$} & $\mathrm{U}^{23}$ & $\mathrm{U}^{13}$ & $\mathrm{U}^{12}$ \\
\hline $\mathrm{Rh}(1)$ & $9(1)$ & $9(1)$ & $7(1)$ & $-2(1)$ & $0(1)$ & $-3(1)$ \\
$\mathrm{Cl}(1)$ & $12(1)$ & $15(1)$ & $9(1)$ & $-5(1)$ & $1(1)$ & $-5(1)$ \\
$\mathrm{S}(1)$ & $14(1)$ & $12(1)$ & $10(1)$ & $-4(1)$ & $3(1)$ & $-4(1)$ \\
$\mathrm{P}(1)$ & $9(1)$ & $10(1)$ & $8(1)$ & $-2(1)$ & $0(1)$ & $-4(1)$ \\
$\mathrm{P}(2)$ & $9(1)$ & $9(1)$ & $9(1)$ & $-3(1)$ & $1(1)$ & $-4(1)$ \\
$\mathrm{O}(1)$ & $15(1)$ & $20(1)$ & $17(1)$ & $0(1)$ & $5(1)$ & $1(1)$
\end{tabular}




\begin{tabular}{|c|c|c|c|c|c|c|}
\hline $\mathrm{O}(2)$ & $33(1)$ & $18(1)$ & $14(1)$ & $-9(1)$ & $4(1)$ & $-14(1)$ \\
\hline $\mathrm{N}(1)$ & $12(1)$ & $13(1)$ & $8(1)$ & $-4(1)$ & $2(1)$ & $-5(1)$ \\
\hline $\mathrm{C}(1)$ & $10(1)$ & $13(1)$ & $8(1)$ & $-4(1)$ & 1(1) & $-5(1)$ \\
\hline$C(2)$ & $17(1)$ & $12(1)$ & $9(1)$ & $-4(1)$ & $3(1)$ & $-5(1)$ \\
\hline$C(3)$ & $16(1)$ & $19(1)$ & $12(1)$ & $-8(1)$ & $2(1)$ & $-7(1)$ \\
\hline $\mathrm{C}(4)$ & $18(1)$ & $17(1)$ & $15(1)$ & $-5(1)$ & $-4(1)$ & $-1(1)$ \\
\hline$C(5)$ & $24(1)$ & $16(1)$ & $13(1)$ & $-2(1)$ & $4(1)$ & $-4(1)$ \\
\hline$C(6)$ & $18(1)$ & $17(1)$ & 21(1) & $-2(1)$ & $3(1)$ & $-8(1)$ \\
\hline$C(7)$ & $14(1)$ & $18(1)$ & $14(1)$ & $-6(1)$ & $2(1)$ & $-6(1)$ \\
\hline $\mathrm{C}(8)$ & $38(2)$ & $24(1)$ & 21(1) & $8(1)$ & $0(1)$ & $-5(1)$ \\
\hline $\mathrm{C}(9)$ & $13(1)$ & $12(1)$ & $8(1)$ & $-3(1)$ & $-1(1)$ & $-5(1)$ \\
\hline $\mathrm{C}(10)$ & $14(1)$ & $12(1)$ & $16(1)$ & $-4(1)$ & 1(1) & $-4(1)$ \\
\hline $\mathrm{C}(11)$ & $22(1)$ & $8(1)$ & $19(1)$ & $-2(1)$ & $-4(1)$ & $-4(1)$ \\
\hline $\mathrm{C}(12)$ & $30(1)$ & $16(1)$ & $19(1)$ & $-3(1)$ & $3(1)$ & $-15(1)$ \\
\hline $\mathrm{C}(13)$ & $18(1)$ & $25(1)$ & 21(1) & $-8(1)$ & $7(1)$ & $-14(1)$ \\
\hline$C(14)$ & 13(1) & $16(1)$ & $16(1)$ & $-6(1)$ & $0(1)$ & $-6(1)$ \\
\hline $\mathrm{C}(15)$ & $12(1)$ & $12(1)$ & $10(1)$ & $-4(1)$ & $0(1)$ & $-2(1)$ \\
\hline $\mathrm{C}(16)$ & $15(1)$ & $16(1)$ & $14(1)$ & $-5(1)$ & $0(1)$ & $-5(1)$ \\
\hline $\mathrm{C}(17)$ & $15(1)$ & $24(1)$ & $18(1)$ & $-6(1)$ & $-3(1)$ & $-7(1)$ \\
\hline $\mathrm{C}(18)$ & $18(1)$ & $24(1)$ & $14(1)$ & $-2(1)$ & $-4(1)$ & $-1(1)$ \\
\hline $\mathrm{C}(19)$ & $25(1)$ & $14(1)$ & $15(1)$ & $-1(1)$ & $0(1)$ & $-3(1)$ \\
\hline $\mathrm{C}(20)$ & $15(1)$ & $15(1)$ & $13(1)$ & $-6(1)$ & 1(1) & $-5(1)$ \\
\hline $\mathrm{C}(22)$ & $15(1)$ & $15(1)$ & $15(1)$ & $-2(1)$ & $-2(1)$ & $-7(1)$ \\
\hline $\mathrm{C}(23)$ & $17(1)$ & $12(1)$ & $24(1)$ & $-2(1)$ & $-2(1)$ & $-5(1)$ \\
\hline $\mathrm{C}(24)$ & $14(1)$ & $15(1)$ & 21(1) & $4(1)$ & $-4(1)$ & $-5(1)$ \\
\hline $\mathrm{C}(25)$ & $15(1)$ & $24(1)$ & $12(1)$ & 1(1) & $-1(1)$ & $-7(1)$ \\
\hline$C(26)$ & $13(1)$ & $18(1)$ & $14(1)$ & $-4(1)$ & 1(1) & $-5(1)$ \\
\hline $\mathrm{C}(27)$ & $8(1)$ & $12(1)$ & $13(1)$ & $-1(1)$ & $0(1)$ & $-4(1)$ \\
\hline $\mathrm{C}(29)$ & $13(1)$ & $16(1)$ & $14(1)$ & $-5(1)$ & $-1(1)$ & $-5(1)$ \\
\hline $\mathrm{C}(30)$ & $11(1)$ & $20(1)$ & 19(1) & $-7(1)$ & $-2(1)$ & $-9(1)$ \\
\hline $\mathrm{C}(31)$ & $19(1)$ & $16(1)$ & $22(1)$ & $-6(1)$ & $2(1)$ & $-10(1)$ \\
\hline $\mathrm{C}(32)$ & $19(1)$ & $14(1)$ & $15(1)$ & $-1(1)$ & $0(1)$ & $-7(1)$ \\
\hline $\mathrm{C}(33)$ & $12(1)$ & $16(1)$ & $13(1)$ & $-5(1)$ & $0(1)$ & $-6(1)$ \\
\hline $\mathrm{C}(34)$ & $11(1)$ & $11(1)$ & $12(1)$ & $-4(1)$ & $3(1)$ & $-5(1)$ \\
\hline $\mathrm{C}(35)$ & $15(1)$ & $10(1)$ & $8(1)$ & $-3(1)$ & $3(1)$ & $-4(1)$ \\
\hline $\mathrm{C}(36)$ & $18(1)$ & $14(1)$ & $15(1)$ & $-6(1)$ & $4(1)$ & $-7(1)$ \\
\hline $\mathrm{C}(37)$ & $32(1)$ & $17(1)$ & $23(1)$ & $-9(1)$ & $7(1)$ & $-15(1)$ \\
\hline $\mathrm{C}(38)$ & $42(2)$ & $12(1)$ & 21(1) & $-5(1)$ & $4(1)$ & $-7(1)$ \\
\hline C(39) & $23(1)$ & $14(1)$ & $17(1)$ & $-2(1)$ & $-2(1)$ & $2(1)$ \\
\hline $\mathrm{C}(40)$ & $19(1)$ & $15(1)$ & $12(1)$ & $-4(1)$ & $0(1)$ & $-4(1)$ \\
\hline Cl11 & $26(1)$ & $28(1)$ & $27(1)$ & $-10(1)$ & $-1(1)$ & $-3(1)$ \\
\hline $\mathrm{Cl} 2 \mathrm{~A} 1$ & $43(1)$ & $46(1)$ & $51(1)$ & $-28(1)$ & $-14(1)$ & $-6(1)$ \\
\hline $\mathrm{Cl3A} 1$ & $84(1)$ & $25(1)$ & $33(1)$ & $-6(1)$ & $35(1)$ & $-1(1)$ \\
\hline C1A1 & $47(4)$ & $14(2)$ & $22(2)$ & $-6(2)$ & $3(2)$ & $-3(2)$ \\
\hline $\mathrm{Cl} 2 \mathrm{~B} 1$ & $82(4)$ & $39(2)$ & $70(3)$ & $10(2)$ & $-50(3)$ & $-29(2)$ \\
\hline $\mathrm{Cl3B} 1$ & $56(3)$ & $48(3)$ & $22(1)$ & $3(2)$ & $11(2)$ & $18(2)$ \\
\hline C1B1 & $48(7)$ & $18(4)$ & $23(4)$ & $-8(4)$ & $-7(4)$ & $-6(4)$ \\
\hline Cl12 & $43(1)$ & $41(1)$ & $68(1)$ & $8(1)$ & $-26(1)$ & $-8(1)$ \\
\hline $\mathrm{Cl} 22$ & $40(1)$ & $25(1)$ & $34(1)$ & $-10(1)$ & 1(1) & $-13(1)$ \\
\hline $\mathrm{Cl} 32$ & $39(1)$ & $36(1)$ & $34(1)$ & $-9(1)$ & $-1(1)$ & $-16(1)$ \\
\hline $\mathrm{C} 12$ & $43(4)$ & $26(3)$ & $42(4)$ & $-9(3)$ & $-18(3)$ & $-14(3)$ \\
\hline
\end{tabular}




\subsection{Crystal Structure Determination of 1}

There are two disordered DCM Molecules in the structure, one of them is on an inversion center. Both of them were modelled using appropriate PART, SAME, SIMU, and RIGU instructions. The final occupancy was 0.63:0.37 and 0.31:0.19.

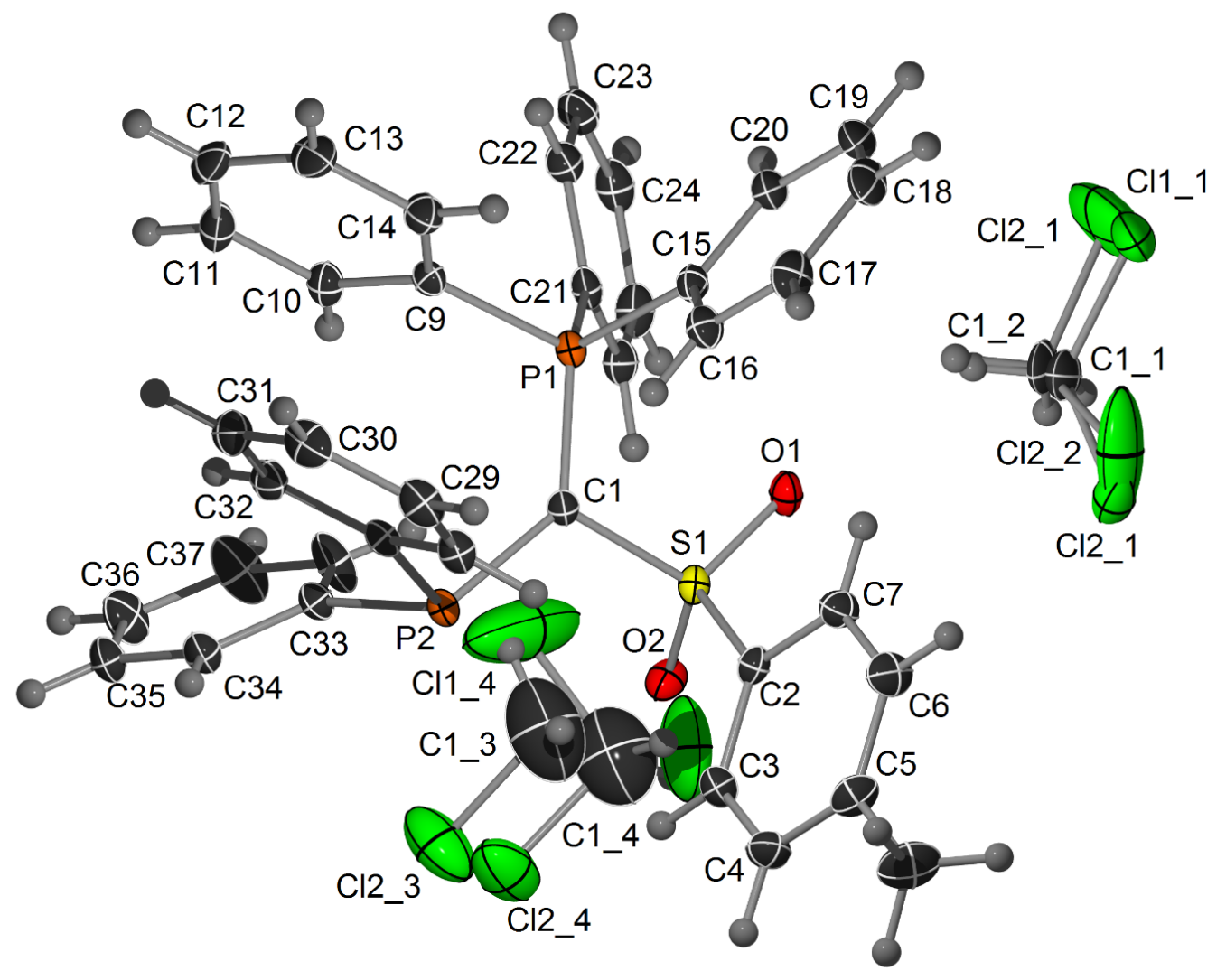

Figure S24. ORTEP Plot of $\mathbf{1}$. Ellipsoids are drawn at the 50\% probability level.

Table S15. Atomic coordinates $\left(\mathrm{x} 10^{4}\right)$ and equivalent isotropic displacement parameters $\left(\AA^{2} \times 10^{3}\right)$ for 1. $U(e q)$ is defined as one third of the trace of the orthogonalized $U^{i j}$ tensor.

\begin{tabular}{lllll}
\hline & $\mathrm{x}$ & $\mathrm{y}$ & $\mathrm{z}$ & $\mathrm{U}(\mathrm{eq})$ \\
\hline $\mathrm{S}(1)$ & $2927(1)$ & $4760(1)$ & $2353(1)$ & $14(1)$ \\
$\mathrm{P}(1)$ & $3900(1)$ & $7067(1)$ & $2052(1)$ & $13(1)$ \\
$\mathrm{P}(2)$ & $902(1)$ & $6901(1)$ & $2767(1)$ & $14(1)$ \\
$\mathrm{O}(1)$ & $4339(1)$ & $4396(1)$ & $2070(1)$ & $19(1)$ \\
$\mathrm{O}(2)$ & $1838(1)$ & $4506(1)$ & $1927(1)$ & $21(1)$ \\
$\mathrm{C}(1)$ & $2581(2)$ & $6331(1)$ & $2383(1)$ & $15(1)$ \\
$\mathrm{C}(2)$ & $2900(2)$ & $3842(1)$ & $3342(1)$ & $16(1)$ \\
$\mathrm{C}(3)$ & $1677(2)$ & $3540(2)$ & $3640(1)$ & $20(1)$ \\
$\mathrm{C}(4)$ & $1672(2)$ & $2790(2)$ & $4401(1)$ & $23(1)$ \\
$\mathrm{C}(5)$ & $2876(2)$ & $2315(2)$ & $4870(1)$ & $22(1)$ \\
$\mathrm{C}(6)$ & $4095(2)$ & $2616(2)$ & $4555(1)$ & $24(1)$ \\
$\mathrm{C}(7)$ & $4113(2)$ & $3380(2)$ & $3799(1)$ & $20(1)$
\end{tabular}




\begin{tabular}{|c|c|c|c|c|}
\hline$C(8)$ & $2869(2)$ & $1494(2)$ & $5694(1)$ & $34(1)$ \\
\hline$C(9)$ & $3431(2)$ & $8700(1)$ & $2158(1)$ & $16(1)$ \\
\hline$C(10)$ & $2488(2)$ & $9583(2)$ & $1606(1)$ & $20(1)$ \\
\hline$C(11)$ & $2147(2)$ & $10849(2)$ & $1649(1)$ & $25(1)$ \\
\hline$C(12)$ & $2749(2)$ & $11250(2)$ & $2232(1)$ & $27(1)$ \\
\hline$C(13)$ & $3691(2)$ & $10381(2)$ & $2775(1)$ & $27(1)$ \\
\hline$C(14)$ & $4040(2)$ & $9105(2)$ & 2742(1) & $22(1)$ \\
\hline$C(15)$ & $5488(2)$ & $6332(1)$ & $2627(1)$ & $16(1)$ \\
\hline$C(16)$ & $5376(2)$ & $6023(2)$ & $3461(1)$ & $20(1)$ \\
\hline$C(17)$ & $6563(2)$ & $5520(2)$ & $3939(1)$ & $24(1)$ \\
\hline$C(18)$ & $7858(2)$ & $5319(2)$ & $3587(1)$ & $26(1)$ \\
\hline$C(19)$ & $7973(2)$ & $5604(2)$ & $2759(1)$ & $24(1)$ \\
\hline$C(20)$ & 6791(2) & $6113(1)$ & $2276(1)$ & $20(1)$ \\
\hline$C(21)$ & $4327(2)$ & $7206(1)$ & $998(1)$ & $16(1)$ \\
\hline$C(22)$ & $5138(2)$ & $8042(2)$ & $653(1)$ & $20(1)$ \\
\hline$C(23)$ & $5514(2)$ & $8149(2)$ & $-146(1)$ & $24(1)$ \\
\hline$C(24)$ & $5088(2)$ & $7426(2)$ & $-608(1)$ & $24(1)$ \\
\hline$C(25)$ & $4263(2)$ & $6617(2)$ & $-276(1)$ & $23(1)$ \\
\hline$C(26)$ & $3875(2)$ & $6506(1)$ & $525(1)$ & 19(1) \\
\hline$C(27)$ & $1159(2)$ & $7824(1)$ & $3492(1)$ & $16(1)$ \\
\hline$C(28)$ & $1762(2)$ & $7110(2)$ & $4226(1)$ & $20(1)$ \\
\hline$C(29)$ & $2008(2)$ & $7699(2)$ & $4826(1)$ & $24(1)$ \\
\hline$C(30)$ & $1620(2)$ & $9011(2)$ & $4707(1)$ & $25(1)$ \\
\hline $\mathrm{C}(31)$ & $991(2)$ & $9726(2)$ & $3989(1)$ & $24(1)$ \\
\hline$C(32)$ & $762(2)$ & $9138(2)$ & $3383(1)$ & 19(1) \\
\hline $\mathrm{C}(33)$ & $-167(2)$ & $8045(1)$ & 1959(1) & $17(1)$ \\
\hline$C(34)$ & $-1430(2)$ & $8806(2)$ & 2141(1) & $20(1)$ \\
\hline$C(35)$ & $-2326(2)$ & $9597(2)$ & $1538(1)$ & $24(1)$ \\
\hline$C(36)$ & $-1989(2)$ & $9632(2)$ & $742(1)$ & $30(1)$ \\
\hline $\mathrm{C}(37)$ & $-763(2)$ & $8853(2)$ & $553(1)$ & $39(1)$ \\
\hline $\mathrm{C}(38)$ & $137(2)$ & $8060(2)$ & $1156(1)$ & $30(1)$ \\
\hline Cl11 & 9196(1) & $2617(1)$ & $2396(1)$ & $46(1)$ \\
\hline $\mathrm{Cl} 21$ & $7019(2)$ & 1472(2) & $3113(1)$ & $36(1)$ \\
\hline $\mathrm{C} 11$ & $7489(6)$ & $2529(12)$ & $2297(6)$ & $32(1)$ \\
\hline Cl12 & $9051(3)$ & $2905(4)$ & 1941(4) & $100(1)$ \\
\hline $\mathrm{Cl} 22$ & $7451(6)$ & 1655(4) & $3141(2)$ & $89(1)$ \\
\hline $\mathrm{C} 12$ & $7390(9)$ & $2750(20)$ & $2235(10)$ & $33(2)$ \\
\hline $\mathrm{Cl13}$ & $1028(4)$ & $3920(3)$ & $-37(2)$ & $86(1)$ \\
\hline $\mathrm{Cl} 23$ & $-1612(2)$ & $5640(5)$ & $169(2)$ & $59(1)$ \\
\hline $\mathrm{C} 13$ & $179(7)$ & $5283(11)$ & 297(9) & $97(3)$ \\
\hline Cl14 & $715(8)$ & $5992(8)$ & $-276(5)$ & $130(3)$ \\
\hline $\mathrm{Cl} 24$ & $-1269(5)$ & $4921(6)$ & $437(2)$ & $55(1)$ \\
\hline C14 & $477(11)$ & $4516(10)$ & $222(14)$ & $97(3)$ \\
\hline
\end{tabular}

Table S16. Anisotropic displacement parameters $\left(\AA^{2} \times 10^{3}\right)$ for 1 . The anisotropic displacement factor exponent takes the form: $-2 \pi^{2}\left[h^{2} a^{* 2} U^{11}+\ldots+2 h k a^{*} b^{*} U^{12}\right]$

\begin{tabular}{lllllll}
\hline & $\mathrm{U}^{11}$ & $\mathrm{U}^{22}$ & $\mathrm{U}^{33}$ & $\mathrm{U}^{23}$ & $\mathrm{U}^{13}$ & $\mathrm{U}^{12}$ \\
\hline $\mathrm{S}(1)$ & $15(1)$ & $13(1)$ & $14(1)$ & $-3(1)$ & $-1(1)$ & $-1(1)$ \\
$\mathrm{P}(1)$ & $12(1)$ & $13(1)$ & $13(1)$ & $-3(1)$ & $-1(1)$ & $-1(1)$ \\
$\mathrm{P}(2)$ & $12(1)$ & $15(1)$ & $15(1)$ & $-3(1)$ & $-1(1)$ & $-1(1)$ \\
$\mathrm{O}(1)$ & $19(1)$ & $17(1)$ & $17(1)$ & $-4(1)$ & $3(1)$ & $1(1)$ \\
$\mathrm{O}(2)$ & $24(1)$ & $18(1)$ & $21(1)$ & $-4(1)$ & $-7(1)$ & $-5(1)$
\end{tabular}




\begin{tabular}{|c|c|c|c|c|c|c|}
\hline $\mathrm{C}(1)$ & $13(1)$ & $14(1)$ & $16(1)$ & $-3(1)$ & $0(1)$ & $-1(1)$ \\
\hline$C(2)$ & $18(1)$ & $13(1)$ & $16(1)$ & $-4(1)$ & $0(1)$ & $-2(1)$ \\
\hline$C(3)$ & $16(1)$ & $20(1)$ & $23(1)$ & $-4(1)$ & $-1(1)$ & $-2(1)$ \\
\hline$C(4)$ & $21(1)$ & $23(1)$ & $25(1)$ & $-5(1)$ & $5(1)$ & $-9(1)$ \\
\hline$C(5)$ & $30(1)$ & $22(1)$ & $17(1)$ & $-3(1)$ & $1(1)$ & $-10(1)$ \\
\hline$C(6)$ & $24(1)$ & $27(1)$ & $21(1)$ & $0(1)$ & $-6(1)$ & $-7(1)$ \\
\hline$C(7)$ & $18(1)$ & $21(1)$ & $20(1)$ & $-2(1)$ & $0(1)$ & $-6(1)$ \\
\hline $\mathrm{C}(8)$ & $44(1)$ & $38(1)$ & $22(1)$ & $2(1)$ & $-1(1)$ & $-20(1)$ \\
\hline $\mathrm{C}(9)$ & $15(1)$ & $15(1)$ & $18(1)$ & $-4(1)$ & $4(1)$ & $-2(1)$ \\
\hline$C(10)$ & $21(1)$ & $19(1)$ & $16(1)$ & $-3(1)$ & $3(1)$ & $-1(1)$ \\
\hline$C(11)$ & $28(1)$ & $18(1)$ & $22(1)$ & $0(1)$ & $8(1)$ & $2(1)$ \\
\hline$C(12)$ & $31(1)$ & $15(1)$ & $34(1)$ & $-8(1)$ & $12(1)$ & $-6(1)$ \\
\hline$C(13)$ & $27(1)$ & $24(1)$ & $37(1)$ & $-15(1)$ & $4(1)$ & $-9(1)$ \\
\hline$C(14)$ & $19(1)$ & $21(1)$ & $26(1)$ & $-8(1)$ & $0(1)$ & $-4(1)$ \\
\hline$C(15)$ & $15(1)$ & $14(1)$ & $18(1)$ & $-3(1)$ & $-4(1)$ & $-1(1)$ \\
\hline$C(16)$ & $18(1)$ & $21(1)$ & $20(1)$ & $-5(1)$ & $-2(1)$ & $-2(1)$ \\
\hline$C(17)$ & $27(1)$ & $24(1)$ & $20(1)$ & $-2(1)$ & $-8(1)$ & $-2(1)$ \\
\hline $\mathrm{C}(18)$ & 19(1) & $23(1)$ & $33(1)$ & $-4(1)$ & $-11(1)$ & $-1(1)$ \\
\hline$C(19)$ & $15(1)$ & 21(1) & $34(1)$ & $-5(1)$ & $-3(1)$ & $-2(1)$ \\
\hline$C(20)$ & $16(1)$ & $18(1)$ & $23(1)$ & $-3(1)$ & $-1(1)$ & $-2(1)$ \\
\hline $\mathrm{C}(21)$ & $14(1)$ & $17(1)$ & $14(1)$ & $-2(1)$ & $0(1)$ & $2(1)$ \\
\hline $\mathrm{C}(22)$ & $17(1)$ & 21(1) & $20(1)$ & $-4(1)$ & 1(1) & $-2(1)$ \\
\hline$C(23)$ & $20(1)$ & $26(1)$ & 21(1) & $-1(1)$ & $4(1)$ & $-2(1)$ \\
\hline$C(24)$ & $24(1)$ & $27(1)$ & $15(1)$ & $-2(1)$ & $2(1)$ & $4(1)$ \\
\hline$C(25)$ & $27(1)$ & $22(1)$ & $17(1)$ & $-6(1)$ & $-3(1)$ & 1(1) \\
\hline$C(26)$ & $20(1)$ & $18(1)$ & $17(1)$ & $-3(1)$ & $-2(1)$ & $0(1)$ \\
\hline$C(27)$ & $13(1)$ & $20(1)$ & $16(1)$ & $-5(1)$ & $3(1)$ & $-3(1)$ \\
\hline $\mathrm{C}(28)$ & $18(1)$ & $22(1)$ & $19(1)$ & $-4(1)$ & $0(1)$ & $-1(1)$ \\
\hline $\mathrm{C}(29)$ & 21(1) & $31(1)$ & $18(1)$ & $-6(1)$ & $-1(1)$ & $-3(1)$ \\
\hline$C(30)$ & $24(1)$ & $33(1)$ & $23(1)$ & $-15(1)$ & $3(1)$ & $-7(1)$ \\
\hline $\mathrm{C}(31)$ & $24(1)$ & 21(1) & $26(1)$ & $-10(1)$ & $4(1)$ & $-4(1)$ \\
\hline$C(32)$ & $18(1)$ & $20(1)$ & $19(1)$ & $-5(1)$ & $3(1)$ & $-2(1)$ \\
\hline $\mathrm{C}(33)$ & $14(1)$ & $19(1)$ & $18(1)$ & $-3(1)$ & $-2(1)$ & $-3(1)$ \\
\hline$C(34)$ & $16(1)$ & $22(1)$ & $20(1)$ & $-4(1)$ & $0(1)$ & $-2(1)$ \\
\hline$C(35)$ & $16(1)$ & $22(1)$ & $29(1)$ & $-3(1)$ & $-4(1)$ & 1(1) \\
\hline$C(36)$ & $22(1)$ & $37(1)$ & $24(1)$ & $3(1)$ & $-9(1)$ & $0(1)$ \\
\hline $\mathrm{C}(37)$ & $27(1)$ & $63(1)$ & $17(1)$ & $-3(1)$ & $-4(1)$ & $2(1)$ \\
\hline$C(38)$ & 19(1) & $43(1)$ & $21(1)$ & $-8(1)$ & $-2(1)$ & $6(1)$ \\
\hline Cl11 & $20(1)$ & $32(1)$ & $83(1)$ & $-15(1)$ & $2(1)$ & $-2(1)$ \\
\hline $\mathrm{Cl} 21$ & $43(1)$ & $22(1)$ & $36(1)$ & $-2(1)$ & $15(1)$ & $-3(1)$ \\
\hline C11 & $24(2)$ & $19(4)$ & $43(2)$ & $5(2)$ & $-6(2)$ & $1(2)$ \\
\hline Cl12 & $39(1)$ & $116(3)$ & $160(4)$ & $-79(3)$ & $41(2)$ & $-14(2)$ \\
\hline $\mathrm{Cl} 22$ & $126(3)$ & $66(2)$ & $28(1)$ & $-3(1)$ & $-5(2)$ & $55(2)$ \\
\hline C12 & $24(3)$ & $17(5)$ & $47(3)$ & $4(2)$ & $-14(2)$ & $5(3)$ \\
\hline Cl13 & $86(2)$ & $62(2)$ & $95(2)$ & $-37(2)$ & $2(2)$ & $26(2)$ \\
\hline Cl23 & $33(1)$ & $91(3)$ & $58(2)$ & $-36(2)$ & $-7(1)$ & $-6(1)$ \\
\hline C13 & $81(6)$ & 113(7) & $91(6)$ & $-40(6)$ & $-10(4)$ & $7(5)$ \\
\hline $\mathrm{Cl} 14$ & $124(5)$ & $119(5)$ & $140(6)$ & $66(5)$ & $-65(4)$ & $-86(5)$ \\
\hline $\mathrm{Cl} 24$ & $40(2)$ & $68(3)$ & $44(2)$ & $10(2)$ & $6(1)$ & $-9(2)$ \\
\hline C14 & $90(6)$ & $109(7)$ & $91(6)$ & $-37(6)$ & $-8(5)$ & $-8(5)$ \\
\hline
\end{tabular}




\subsection{Crystal Structure Determination of 3}

The structure is pseudo- $A$-centered. The reflections that would be absent in a true $A$-centering are systematically weak but not absent. The solution without centering was preferred due to better R-values and much more reasonable ADPs. There are two THF molecules, each disordered over two positions. They were modelled using appropriate PART, SAME and RIGU instructions. The final occupancy was 0.94:0.06 for both.

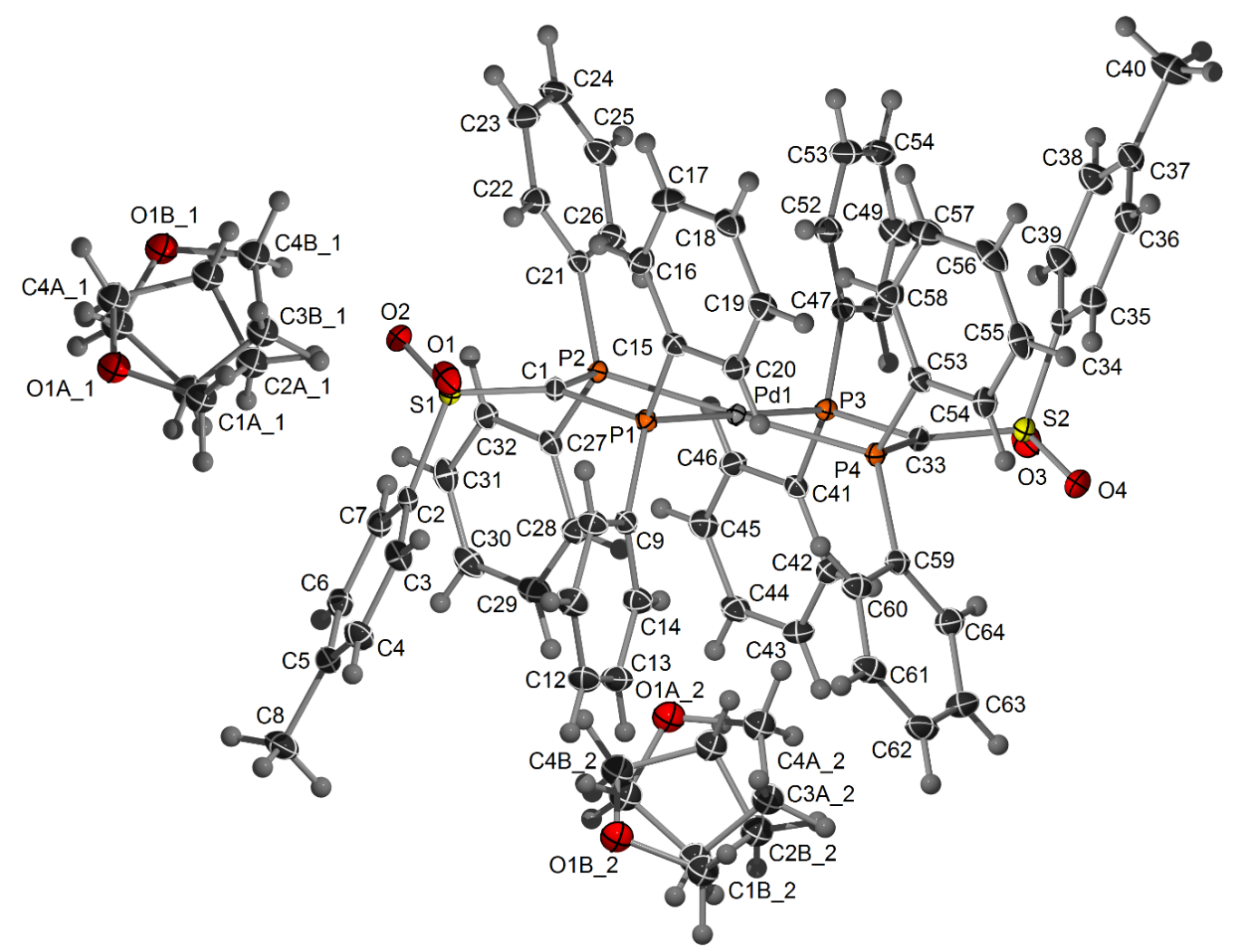

Figure S25. ORTEP Plot of 3. Ellipsoids are drawn at the 50\% probability level.

Table S17. Atomic coordinates $\left(\mathrm{x} 10^{4}\right)$ and equivalent isotropic displacement parameters $\left(\AA^{2} \times 10^{3}\right)$ for 3. $U(e q)$ is defined as one third of the trace of the orthogonalized $U^{i j}$ tensor.

\begin{tabular}{lrrll}
\hline & $\mathrm{x}$ & $\mathrm{y}$ & $\mathrm{Z}$ & $\mathrm{U}(\mathrm{eq})$ \\
\hline $\mathrm{Pd}(1)$ & $5088(1)$ & $2530(1)$ & $2473(1)$ & $10(1)$ \\
$\mathrm{S}(1)$ & $3838(1)$ & $2788(1)$ & $4565(1)$ & $15(1)$ \\
$\mathrm{S}(2)$ & $6408(1)$ & $2193(1)$ & $396(1)$ & $16(1)$ \\
$\mathrm{P}(1)$ & $3589(1)$ & $2299(1)$ & $3247(1)$ & $11(1)$ \\
$\mathrm{P}(2)$ & $5708(1)$ & $2726(1)$ & $3422(1)$ & $11(1)$ \\
$\mathrm{P}(3)$ & $6581(1)$ & $2772(1)$ & $1702(1)$ & $11(1)$ \\
$\mathrm{P}(4)$ & $4497(1)$ & $2294(1)$ & $1527(1)$ & $12(1)$ \\
$\mathrm{O}(1)$ & $2955(1)$ & $2180(1)$ & $4768(1)$ & $24(1)$ \\
$\mathrm{O}(2)$ & $4847(1)$ & $2633(1)$ & $4943(1)$ & $24(1)$ \\
$\mathrm{O}(3)$ & $7325(1)$ & $2767(1)$ & $203(1)$ & $25(1)$ \\
$\mathrm{O}(4)$ & $5437(1)$ & $2346(1)$ & $-6(1)$ & $26(1)$ \\
$\mathrm{C}(1)$ & $4376(1)$ & $2550(1)$ & $3829(1)$ & $13(1)$ \\
$\mathrm{C}(2)$ & $3062(1)$ & $4184(1)$ & $4549(1)$ & $15(1)$
\end{tabular}




\begin{tabular}{|c|c|c|c|c|}
\hline$C(3)$ & $1817(1)$ & 4494(1) & 4581(1) & $19(1)$ \\
\hline $\mathrm{C}(4)$ & $1221(1)$ & $5571(1)$ & $4625(1)$ & $21(1)$ \\
\hline$C(5)$ & 1854(1) & $6346(1)$ & $4636(1)$ & $20(1)$ \\
\hline$C(6)$ & $3100(1)$ & $6021(1)$ & $4588(1)$ & $18(1)$ \\
\hline$C(7)$ & $3709(1)$ & 4947(1) & $4545(1)$ & $17(1)$ \\
\hline $\mathrm{C}(8)$ & $1197(2)$ & 7503(1) & 4712(1) & $27(1)$ \\
\hline$C(9)$ & 2089(1) & $3214(1)$ & $3218(1)$ & $14(1)$ \\
\hline$C(10)$ & 1119(1) & 2921(1) & $3569(1)$ & $18(1)$ \\
\hline$C(11)$ & $-6(1)$ & $3657(1)$ & $3575(1)$ & $22(1)$ \\
\hline$C(12)$ & $-167(1)$ & 4692(1) & $3233(1)$ & $22(1)$ \\
\hline$C(13)$ & 794(1) & 4988(1) & 2886(1) & $23(1)$ \\
\hline$C(14)$ & 1920(1) & $4254(1)$ & $2878(1)$ & $19(1)$ \\
\hline$C(15)$ & $3346(1)$ & $944(1)$ & 3386(1) & $14(1)$ \\
\hline$C(16)$ & $4010(1)$ & $134(1)$ & $3814(1)$ & 19(1) \\
\hline$C(17)$ & $3900(1)$ & $-928(1)$ & 3881(1) & $23(1)$ \\
\hline$C(18)$ & $3124(1)$ & $-1181(1)$ & $3521(1)$ & $22(1)$ \\
\hline$C(19)$ & $2448(1)$ & $-375(1)$ & $3097(1)$ & $20(1)$ \\
\hline$C(20)$ & $2564(1)$ & $682(1)$ & $3026(1)$ & $17(1)$ \\
\hline$C(21)$ & 7032(1) & $1768(1)$ & $3759(1)$ & $14(1)$ \\
\hline$C(22)$ & $6935(1)$ & 992(1) & $4277(1)$ & 19(1) \\
\hline$C(23)$ & $7960(1)$ & $247(1)$ & 4514(1) & $23(1)$ \\
\hline$C(24)$ & 9081(1) & $274(1)$ & 4232(1) & $24(1)$ \\
\hline$C(25)$ & $9188(1)$ & $1035(1)$ & 3712(1) & $23(1)$ \\
\hline$C(26)$ & $8164(1)$ & 1781(1) & $3475(1)$ & $18(1)$ \\
\hline$C(27)$ & $5907(1)$ & $4069(1)$ & $3430(1)$ & $14(1)$ \\
\hline$C(28)$ & $5195(1)$ & 4944(1) & $3053(1)$ & $18(1)$ \\
\hline$C(29)$ & $5217(1)$ & $6000(1)$ & $3090(1)$ & $21(1)$ \\
\hline$C(30)$ & $5961(1)$ & $6187(1)$ & $3500(1)$ & $22(1)$ \\
\hline $\mathrm{C}(31)$ & $6690(1)$ & $5324(1)$ & 3863(1) & 19(1) \\
\hline$C(32)$ & $6669(1)$ & $4261(1)$ & $3832(1)$ & $16(1)$ \\
\hline $\mathrm{C}(33)$ & $5823(1)$ & 2489(1) & 1118(1) & $14(1)$ \\
\hline$C(34)$ & $7150(1)$ & 783(1) & $451(1)$ & $16(1)$ \\
\hline$C(35)$ & 6497(1) & $40(1)$ & $420(1)$ & 19(1) \\
\hline$C(36)$ & 7093(1) & $-1052(1)$ & $418(1)$ & $21(1)$ \\
\hline$C(37)$ & $8330(1)$ & $-1411(1)$ & $447(1)$ & $23(1)$ \\
\hline $\mathrm{C}(38)$ & $8966(1)$ & $-655(1)$ & $494(1)$ & $26(1)$ \\
\hline$C(39)$ & $8384(1)$ & $437(1)$ & $494(1)$ & $23(1)$ \\
\hline$C(40)$ & $8990(2)$ & $-2585(1)$ & $420(1)$ & $34(1)$ \\
\hline $\mathrm{C}(41)$ & $6797(1)$ & $4137(1)$ & $1574(1)$ & $14(1)$ \\
\hline$C(42)$ & $6127(1)$ & $4952(1)$ & 1151(1) & $18(1)$ \\
\hline C(43) & $6214(1)$ & $6017(1)$ & 1106(1) & $22(1)$ \\
\hline$C(44)$ & $6968(1)$ & $6267(1)$ & $1480(1)$ & $20(1)$ \\
\hline$C(45)$ & $7650(1)$ & $5455(1)$ & 1899(1) & 19(1) \\
\hline$C(46)$ & $7559(1)$ & 4394(1) & 1950(1) & $17(1)$ \\
\hline C(47) & $8097(1)$ & 1893(1) & 1744(1) & $15(1)$ \\
\hline C(48) & $9056(1)$ & 2193(1) & 1386(1) & $18(1)$ \\
\hline C(49) & 10193(1) & $1480(1)$ & 1394(1) & $21(1)$ \\
\hline$C(50)$ & 10381(1) & $464(1)$ & $1758(1)$ & $22(1)$ \\
\hline $\mathrm{C}(51)$ & $9430(1)$ & $158(1)$ & 2113(1) & $24(1)$ \\
\hline$C(52)$ & 8291(1) & $871(1)$ & $2107(1)$ & 19(1) \\
\hline C(53) & $4338(1)$ & $926(1)$ & $1550(1)$ & $15(1)$ \\
\hline C(54) & $3605(1)$ & $680(1)$ & $1155(1)$ & $18(1)$ \\
\hline C(55) & $3588(1)$ & $-396(1)$ & 1162(1) & $22(1)$ \\
\hline$C(56)$ & $4288(1)$ & $-1223(1)$ & 1559(1) & $24(1)$ \\
\hline $\mathrm{C}(57)$ & $5005(1)$ & $-980(1)$ & 1962(1) & $23(1)$ \\
\hline
\end{tabular}




\begin{tabular}{lllll}
$\mathrm{C}(58)$ & $5029(1)$ & $89(1)$ & $1957(1)$ & $19(1)$ \\
$\mathrm{C}(59)$ & $3149(1)$ & $3197(1)$ & $1187(1)$ & $15(1)$ \\
$\mathrm{C}(60)$ & $2048(1)$ & $3224(1)$ & $1517(1)$ & $19(1)$ \\
$\mathrm{C}(61)$ & $997(1)$ & $3930(1)$ & $1285(1)$ & $23(1)$ \\
$\mathrm{C}(62)$ & $1044(1)$ & $4609(1)$ & $724(1)$ & $24(1)$ \\
$\mathrm{C}(63)$ & $2138(1)$ & $4595(1)$ & $395(1)$ & $26(1)$ \\
$\mathrm{C}(64)$ & $3193(1)$ & $3894(1)$ & $626(1)$ & $21(1)$ \\
$\mathrm{O} 1 \mathrm{~A} 1$ & $820(1)$ & $2386(1)$ & $7243(1)$ & $27(1)$ \\
$\mathrm{C} 1 \mathrm{~A} 1$ & $567(2)$ & $2368(1)$ & $6612(1)$ & $28(1)$ \\
$\mathrm{C} 2 \mathrm{~A} 1$ & $1766(2)$ & $2187(2)$ & $6241(1)$ & $30(1)$ \\
$\mathrm{C} 3 \mathrm{~A} 1$ & $2587(2)$ & $1437(1)$ & $6727(1)$ & $29(1)$ \\
$\mathrm{C} 4 \mathrm{~A} 1$ & $2083(2)$ & $1929(2)$ & $7317(1)$ & $26(1)$ \\
$\mathrm{O} 1 \mathrm{~B} 1$ & $3074(17)$ & $1450(15)$ & $7048(9)$ & $35(2)$ \\
$\mathrm{C} 1 \mathrm{~B} 1$ & $2050(30)$ & $2290(20)$ & $7198(12)$ & $28(3)$ \\
$\mathrm{C} 2 \mathrm{~B} 1$ & $1390(20)$ & $2691(18)$ & $6594(11)$ & $32(2)$ \\
$\mathrm{C} 3 \mathrm{~B} 1$ & $1750(20)$ & $1670(20)$ & $6293(13)$ & $34(3)$ \\
$\mathrm{C} 4 \mathrm{~B} 1$ & $3070(20)$ & $1170(20)$ & $6437(12)$ & $37(3)$ \\
$\mathrm{O} 1 \mathrm{~A} 2$ & $3194(1)$ & $6436(1)$ & $2060(1)$ & $35(1)$ \\
$\mathrm{C} 1 \mathrm{~A} 2$ & $2194(2)$ & $7319(2)$ & $2191(1)$ & $32(1)$ \\
$\mathrm{C} 2 \mathrm{~A} 2$ & $1470(2)$ & $7653(1)$ & $1618(1)$ & $31(1)$ \\
$\mathrm{C} 3 \mathrm{~A} 2$ & $1752(2)$ & $6606(1)$ & $1336(1)$ & $26(1)$ \\
$\mathrm{C} 4 \mathrm{~A} 2$ & $3049(2)$ & $6121(1)$ & $1475(1)$ & $29(1)$ \\
$\mathrm{O} 1 \mathrm{~B} 2$ & $956(12)$ & $7412(13)$ & $2274(5)$ & $31(4)$ \\
$\mathrm{C} 1 \mathrm{~B} 2$ & $691(11)$ & $7447(17)$ & $1641(6)$ & $31(3)$ \\
$\mathrm{C} 2 \mathrm{~B} 2$ & $1878(14)$ & $7181(17)$ & $1269(5)$ & $27(2)$ \\
$\mathrm{C} 3 \mathrm{~B} 2$ & $2715(12)$ & $6469(16)$ & $1756(7)$ & $32(2)$ \\
$\mathrm{C} 4 \mathrm{~B} 2$ & $2225(13)$ & $6993(18)$ & $2331(6)$ & $31(3)$ \\
\hline
\end{tabular}

Table S18. Anisotropic displacement parameters $\left(\AA^{2} \times 10^{3}\right)$ for 3. The anisotropic displacement factor exponent takes the form: $-2 \pi^{2}\left[h^{2} a^{* 2} U^{11}+\ldots+2 h k a^{*} b^{*} U^{12}\right]$

\begin{tabular}{lllllll}
\hline & $\mathrm{U}^{11}$ & $\mathrm{U}^{22}$ & $\mathrm{U}^{33}$ & $\mathrm{U}^{23}$ & $\mathrm{U}^{13}$ & \multicolumn{1}{c}{$\mathrm{U}^{12}$} \\
\hline $\mathrm{Pd}(1)$ & $10(1)$ & $10(1)$ & $10(1)$ & $-2(1)$ & $-1(1)$ & $-3(1)$ \\
$\mathrm{S}(1)$ & $19(1)$ & $13(1)$ & $11(1)$ & $-2(1)$ & $0(1)$ & $-3(1)$ \\
$\mathrm{S}(2)$ & $21(1)$ & $15(1)$ & $11(1)$ & $-2(1)$ & $-1(1)$ & $-3(1)$ \\
$\mathrm{P}(1)$ & $11(1)$ & $11(1)$ & $12(1)$ & $-2(1)$ & $-1(1)$ & $-3(1)$ \\
$\mathrm{P}(2)$ & $12(1)$ & $11(1)$ & $11(1)$ & $-2(1)$ & $-2(1)$ & $-2(1)$ \\
$\mathrm{P}(3)$ & $11(1)$ & $11(1)$ & $12(1)$ & $-2(1)$ & $-1(1)$ & $-3(1)$ \\
$\mathrm{P}(4)$ & $13(1)$ & $12(1)$ & $12(1)$ & $-2(1)$ & $-3(1)$ & $-3(1)$ \\
$\mathrm{O}(1)$ & $32(1)$ & $18(1)$ & $22(1)$ & $-4(1)$ & $10(1)$ & $-9(1)$ \\
$\mathrm{O}(2)$ & $30(1)$ & $22(1)$ & $17(1)$ & $-5(1)$ & $-10(1)$ & $3(1)$ \\
$\mathrm{O}(3)$ & $33(1)$ & $20(1)$ & $21(1)$ & $-2(1)$ & $9(1)$ & $-10(1)$ \\
$\mathrm{O}(4)$ & $33(1)$ & $24(1)$ & $17(1)$ & $-5(1)$ & $-10(1)$ & $3(1)$ \\
$\mathrm{C}(1)$ & $13(1)$ & $14(1)$ & $11(1)$ & $-3(1)$ & $-1(1)$ & $-3(1)$ \\
$\mathrm{C}(2)$ & $18(1)$ & $14(1)$ & $11(1)$ & $-4(1)$ & $0(1)$ & $-2(1)$ \\
$\mathrm{C}(3)$ & $18(1)$ & $20(1)$ & $21(1)$ & $-7(1)$ & $1(1)$ & $-6(1)$ \\
$\mathrm{C}(4)$ & $16(1)$ & $22(1)$ & $24(1)$ & $-7(1)$ & $1(1)$ & $-2(1)$ \\
$\mathrm{C}(5)$ & $24(1)$ & $17(1)$ & $16(1)$ & $-4(1)$ & $0(1)$ & $-2(1)$ \\
$\mathrm{C}(6)$ & $24(1)$ & $17(1)$ & $16(1)$ & $-3(1)$ & $0(1)$ & $-8(1)$ \\
$\mathrm{C}(7)$ & $16(1)$ & $20(1)$ & $15(1)$ & $-5(1)$ & $0(1)$ & $-4(1)$ \\
$\mathrm{C}(8)$ & $29(1)$ & $17(1)$ & $32(1)$ & $-6(1)$ & $1(1)$ & $-1(1)$ \\
$\mathrm{C}(9)$ & $13(1)$ & $15(1)$ & $14(1)$ & $-4(1)$ & $-2(1)$ & $-2(1)$ \\
$\mathrm{C}(10)$ & $16(1)$ & $16(1)$ & $21(1)$ & $-2(1)$ & $0(1)$ & $-4(1)$
\end{tabular}




\begin{tabular}{|c|c|c|c|c|c|c|}
\hline$C(11)$ & $14(1)$ & $24(1)$ & $26(1)$ & $-5(1)$ & $1(1)$ & $-4(1)$ \\
\hline$C(12)$ & $16(1)$ & $22(1)$ & $25(1)$ & $-5(1)$ & $-3(1)$ & $2(1)$ \\
\hline$C(13)$ & $24(1)$ & $18(1)$ & 23(1) & $0(1)$ & $-1(1)$ & 1(1) \\
\hline$C(14)$ & $17(1)$ & $18(1)$ & $20(1)$ & $-2(1)$ & 1(1) & $-3(1)$ \\
\hline$C(15)$ & $14(1)$ & 13(1) & $15(1)$ & $-3(1)$ & 2(1) & $-3(1)$ \\
\hline$C(16)$ & $20(1)$ & $18(1)$ & $19(1)$ & $-3(1)$ & $-6(1)$ & $-4(1)$ \\
\hline$C(17)$ & $28(1)$ & $16(1)$ & 23(1) & 2(1) & $-5(1)$ & $-2(1)$ \\
\hline $\mathrm{C}(18)$ & $27(1)$ & $14(1)$ & $24(1)$ & $-2(1)$ & 1(1) & $-7(1)$ \\
\hline C(19) & $20(1)$ & $18(1)$ & $24(1)$ & $-6(1)$ & $-2(1)$ & $-7(1)$ \\
\hline$C(20)$ & $18(1)$ & $15(1)$ & $18(1)$ & $-1(1)$ & $-3(1)$ & $-4(1)$ \\
\hline$C(21)$ & $15(1)$ & $12(1)$ & $17(1)$ & $-6(1)$ & $-4(1)$ & $-2(1)$ \\
\hline $\mathrm{C}(22)$ & $19(1)$ & $16(1)$ & 21(1) & $-2(1)$ & $-4(1)$ & $-4(1)$ \\
\hline$C(23)$ & $28(1)$ & $16(1)$ & $25(1)$ & $0(1)$ & $-7(1)$ & $-2(1)$ \\
\hline$C(24)$ & $21(1)$ & $17(1)$ & $33(1)$ & $-8(1)$ & $-12(1)$ & $4(1)$ \\
\hline$C(25)$ & $15(1)$ & $22(1)$ & $32(1)$ & $-9(1)$ & $-4(1)$ & $-2(1)$ \\
\hline$C(26)$ & $17(1)$ & $17(1)$ & 21(1) & $-4(1)$ & $-2(1)$ & $-3(1)$ \\
\hline$C(27)$ & $14(1)$ & $14(1)$ & $15(1)$ & $-4(1)$ & 2(1) & $-5(1)$ \\
\hline$C(28)$ & $18(1)$ & $17(1)$ & $18(1)$ & $-2(1)$ & $-2(1)$ & $-4(1)$ \\
\hline$C(29)$ & $22(1)$ & $15(1)$ & $25(1)$ & $0(1)$ & $0(1)$ & $-4(1)$ \\
\hline$C(30)$ & $25(1)$ & $15(1)$ & $28(1)$ & $-6(1)$ & $5(1)$ & $-9(1)$ \\
\hline$C(31)$ & $20(1)$ & $22(1)$ & $20(1)$ & $-8(1)$ & $3(1)$ & $-11(1)$ \\
\hline$C(32)$ & $15(1)$ & $17(1)$ & $16(1)$ & $-4(1)$ & $0(1)$ & $-5(1)$ \\
\hline$C(33)$ & $14(1)$ & $15(1)$ & $12(1)$ & $-2(1)$ & $-1(1)$ & $-4(1)$ \\
\hline$C(34)$ & 21(1) & $17(1)$ & 11(1) & $-4(1)$ & 1(1) & $-3(1)$ \\
\hline$C(35)$ & $20(1)$ & 21(1) & $17(1)$ & $-5(1)$ & $-1(1)$ & $-6(1)$ \\
\hline$C(36)$ & $26(1)$ & $20(1)$ & $19(1)$ & $-4(1)$ & $2(1)$ & $-9(1)$ \\
\hline C(37) & $28(1)$ & $18(1)$ & 21(1) & $-6(1)$ & $2(1)$ & $-3(1)$ \\
\hline C(38) & $19(1)$ & $25(1)$ & $36(1)$ & $-11(1)$ & $-2(1)$ & $-2(1)$ \\
\hline C(39) & 21(1) & $22(1)$ & $28(1)$ & $-10(1)$ & $0(1)$ & $-6(1)$ \\
\hline C(40) & $32(1)$ & $21(1)$ & $45(1)$ & $-9(1)$ & $2(1)$ & $-1(1)$ \\
\hline C(41) & $14(1)$ & $13(1)$ & $15(1)$ & $-4(1)$ & $1(1)$ & $-2(1)$ \\
\hline C(42) & $19(1)$ & $17(1)$ & $17(1)$ & $-3(1)$ & $-4(1)$ & $-3(1)$ \\
\hline C(43) & $26(1)$ & $15(1)$ & $21(1)$ & 1(1) & $-5(1)$ & $-1(1)$ \\
\hline C(44) & $25(1)$ & $12(1)$ & $23(1)$ & $-2(1)$ & 1(1) & $-5(1)$ \\
\hline C(45) & $20(1)$ & $18(1)$ & $23(1)$ & $-4(1)$ & $-3(1)$ & $-7(1)$ \\
\hline C(46) & $18(1)$ & $15(1)$ & $18(1)$ & $-1(1)$ & $-4(1)$ & $-3(1)$ \\
\hline C(47) & $15(1)$ & $15(1)$ & $15(1)$ & $-4(1)$ & $-2(1)$ & $-3(1)$ \\
\hline C(48) & $17(1)$ & $15(1)$ & $22(1)$ & $-2(1)$ & $-1(1)$ & $-4(1)$ \\
\hline C(49) & $15(1)$ & $22(1)$ & $28(1)$ & $-6(1)$ & 1(1) & $-6(1)$ \\
\hline C(50) & $17(1)$ & $20(1)$ & $28(1)$ & $-6(1)$ & $-4(1)$ & $2(1)$ \\
\hline $\mathrm{C}(51)$ & $25(1)$ & $17(1)$ & $25(1)$ & $0(1)$ & $-3(1)$ & $0(1)$ \\
\hline $\mathrm{C}(52)$ & $19(1)$ & $16(1)$ & $19(1)$ & $-1(1)$ & $0(1)$ & $-3(1)$ \\
\hline $\mathrm{C}(53)$ & $17(1)$ & $14(1)$ & $16(1)$ & $-4(1)$ & 2(1) & $-5(1)$ \\
\hline C(54) & $20(1)$ & $20(1)$ & $17(1)$ & $-5(1)$ & $0(1)$ & $-8(1)$ \\
\hline $\mathrm{C}(55)$ & $25(1)$ & $25(1)$ & $22(1)$ & $-10(1)$ & $4(1)$ & $-14(1)$ \\
\hline C(56) & $27(1)$ & $16(1)$ & $32(1)$ & $-9(1)$ & $7(1)$ & $-10(1)$ \\
\hline C(57) & $24(1)$ & $15(1)$ & $29(1)$ & $-1(1)$ & $0(1)$ & $-4(1)$ \\
\hline C(58) & $20(1)$ & $17(1)$ & 21(1) & $-3(1)$ & $-3(1)$ & $-5(1)$ \\
\hline C(59) & $17(1)$ & $12(1)$ & $18(1)$ & $-4(1)$ & $-5(1)$ & $-4(1)$ \\
\hline $\mathrm{C}(60)$ & $19(1)$ & $16(1)$ & $23(1)$ & $-3(1)$ & $-3(1)$ & $-5(1)$ \\
\hline $\mathrm{C}(61)$ & $17(1)$ & $19(1)$ & $33(1)$ & $-7(1)$ & $-5(1)$ & $-3(1)$ \\
\hline $\mathrm{C}(62)$ & $22(1)$ & $18(1)$ & $32(1)$ & $-7(1)$ & $-12(1)$ & $2(1)$ \\
\hline $\mathrm{C}(63)$ & $29(1)$ & $21(1)$ & $24(1)$ & 1(1) & $-8(1)$ & $0(1)$ \\
\hline $\mathrm{C}(64)$ & 21(1) & $19(1)$ & 21(1) & $-2(1)$ & $-3(1)$ & $-4(1)$ \\
\hline O1A1 & $25(1)$ & $29(1)$ & 21(1) & $-2(1)$ & $0(1)$ & 1(1) \\
\hline
\end{tabular}




\begin{tabular}{lllllll} 
C1A1 & $33(1)$ & $27(1)$ & $22(1)$ & $0(1)$ & $-3(1)$ & $-7(1)$ \\
C2A1 & $46(1)$ & $22(1)$ & $24(1)$ & $-6(1)$ & $10(1)$ & $-16(1)$ \\
C3A1 & $26(1)$ & $20(1)$ & $41(1)$ & $-7(1)$ & $9(1)$ & $-8(1)$ \\
C4A1 & $25(1)$ & $20(1)$ & $32(1)$ & $-3(1)$ & $-4(1)$ & $-5(1)$ \\
O1B1 & $38(4)$ & $21(4)$ & $45(4)$ & $-7(4)$ & $3(4)$ & $-7(4)$ \\
C1B1 & $32(5)$ & $21(5)$ & $30(4)$ & $-3(4)$ & $-3(4)$ & $-3(5)$ \\
C2B1 & $40(4)$ & $27(3)$ & $27(3)$ & $-3(3)$ & $3(3)$ & $-9(3)$ \\
C3B1 & $46(4)$ & $27(5)$ & $28(5)$ & $-6(4)$ & $9(4)$ & $-12(5)$ \\
C4B1 & $37(4)$ & $26(5)$ & $43(5)$ & $-6(4)$ & $10(4)$ & $-5(4)$ \\
O1A2 & $42(1)$ & $32(1)$ & $32(1)$ & $-9(1)$ & $-11(1)$ & $-4(1)$ \\
C1A2 & $38(1)$ & $34(1)$ & $32(1)$ & $-17(1)$ & $9(1)$ & $-19(1)$ \\
C2A2 & $26(1)$ & $25(1)$ & $40(1)$ & $-10(1)$ & $6(1)$ & $-6(1)$ \\
C3A2 & $32(1)$ & $28(1)$ & $23(1)$ & $-6(1)$ & $2(1)$ & $-15(1)$ \\
C4A2 & $38(1)$ & $22(1)$ & $26(1)$ & $-7(1)$ & $-1(1)$ & $-2(1)$ \\
O1B2 & $42(7)$ & $19(8)$ & $24(6)$ & $2(7)$ & $2(5)$ & $2(6)$ \\
C1B2 & $31(4)$ & $25(5)$ & $35(4)$ & $-7(4)$ & $4(4)$ & $-4(4)$ \\
C2B2 & $30(3)$ & $24(4)$ & $30(3)$ & $-9(3)$ & $1(3)$ & $-11(3)$ \\
C3B2 & $40(3)$ & $26(4)$ & $29(3)$ & $-9(3)$ & $-2(3)$ & $-4(3)$ \\
C4B2 & $40(4)$ & $33(5)$ & $32(4)$ & $-18(4)$ & $-1(4)$ & $-19(5)$ \\
\hline
\end{tabular}




\subsection{Crystal Structure Determination of $[4 a]\left[\mathrm{BF}_{4}\right]$}

The $\mathrm{CH}_{2}$-Atoms of the COD-ligand are disordered over two positions. The disorder was modelled using appropriate PART, SIMU and RIGU instructions. The BF 4 -Anion was disordered over two positions, which was modelled using appropriate PART, SADI, SIMU and RIGU instructions. The final occupancy of the two possible positions of both was 0.59:0.41.

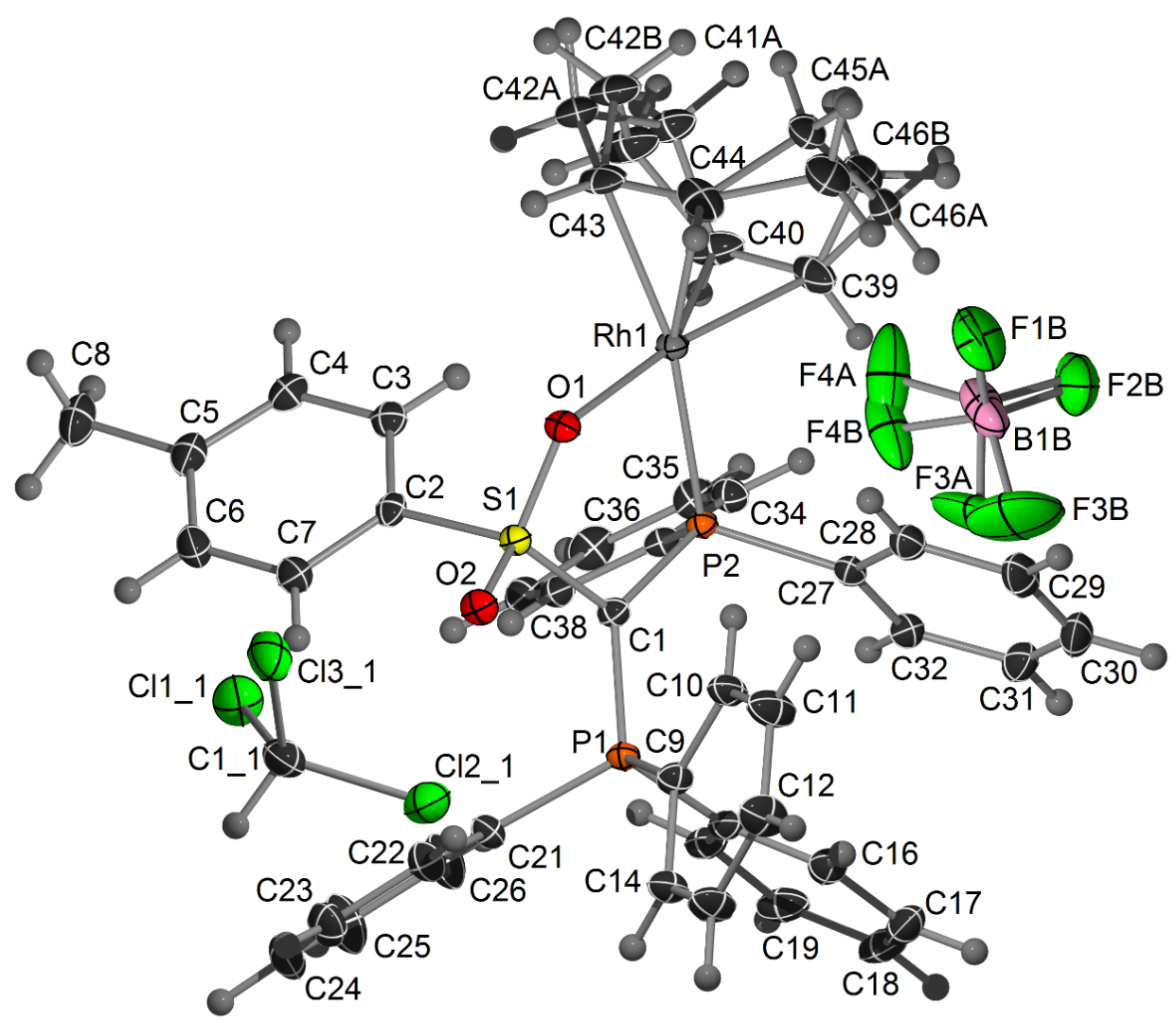

Figure S26. ORTEP Plot of $[4 \mathrm{a}]\left[\mathrm{BF}_{4}\right]$. Ellipsoids are drawn at the $50 \%$ probability level.

Table S19. Atomic coordinates ( $\left.\mathrm{x} 10^{4}\right)$ and equivalent isotropic displacement parameters $\left(\AA^{2} \times 10^{3}\right)$ for $[\mathbf{4 a}]\left[\mathrm{BF}_{4}\right]$. $\mathrm{U}(\mathrm{eq})$ is defined as one third of the trace of the orthogonalized $\mathrm{Uij}^{\mathrm{ij}}$ tensor.

\begin{tabular}{lrrrr}
\hline & $\mathrm{x}$ & $\mathrm{y}$ & $\mathrm{z}$ & $\mathrm{U}(\mathrm{eq})$ \\
\hline $\mathrm{Rh}(1)$ & $4334(1)$ & $6258(1)$ & $5703(1)$ & $13(1)$ \\
$\mathrm{S}(1)$ & $4110(1)$ & $3919(1)$ & $5324(1)$ & $13(1)$ \\
$\mathrm{P}(1)$ & $3345(1)$ & $3569(1)$ & $5601(1)$ & $13(1)$ \\
$\mathrm{P}(2)$ & $3827(1)$ & $5561(1)$ & $6186(1)$ & $12(1)$ \\
$\mathrm{O}(1)$ & $4308(1)$ & $4878(1)$ & $5098(1)$ & $16(1)$ \\
$\mathrm{O}(2)$ & $3981(1)$ & $3185(1)$ & $4804(1)$ & $18(1)$ \\
$\mathrm{C}(1)$ & $3748(1)$ & $4329(1)$ & $5756(1)$ & $14(1)$ \\
$\mathrm{C}(2)$ & $4452(1)$ & $3228(1)$ & $5836(1)$ & $16(1)$ \\
$\mathrm{C}(3)$ & $4711(1)$ & $3785(1)$ & $6252(1)$ & $18(1)$ \\
$\mathrm{C}(4)$ & $4992(1)$ & $3228(1)$ & $6612(1)$ & $21(1)$ \\
$\mathrm{C}(5)$ & $5020(1)$ & $2119(1)$ & $6558(1)$ & $23(1)$ \\
$\mathrm{C}(6)$ & $4752(1)$ & $1579(1)$ & $6155(1)$ & $26(1)$
\end{tabular}




\begin{tabular}{|c|c|c|c|c|}
\hline$C(7)$ & $4466(1)$ & $2120(1)$ & $5793(1)$ & $21(1)$ \\
\hline $\mathrm{C}(8)$ & $5341(1)$ & $1522(2)$ & 6913(1) & $32(1)$ \\
\hline C(9) & $3150(1)$ & $3700(1)$ & $4752(1)$ & $16(1)$ \\
\hline $\mathrm{C}(10)$ & $3264(1)$ & 4531(1) & $4359(1)$ & $21(1)$ \\
\hline $\mathrm{C}(11)$ & $3102(1)$ & $4658(1)$ & 3711(1) & $27(1)$ \\
\hline$C(12)$ & $2827(1)$ & $3957(1)$ & $3465(1)$ & $28(1)$ \\
\hline$C(13)$ & 2709(1) & $3134(1)$ & $3860(1)$ & $24(1)$ \\
\hline$C(14)$ & $2869(1)$ & 3001(1) & $4502(1)$ & $20(1)$ \\
\hline$C(15)$ & 2986(1) & 4048(1) & $6112(1)$ & $17(1)$ \\
\hline$C(16)$ & 2669(1) & 4533(1) & $5797(1)$ & $22(1)$ \\
\hline$C(17)$ & $2379(1)$ & $4855(1)$ & $6168(1)$ & $29(1)$ \\
\hline$C(18)$ & $2398(1)$ & $4679(2)$ & $6846(1)$ & $30(1)$ \\
\hline$C(19)$ & $2712(1)$ & 4199(1) & $7163(1)$ & $26(1)$ \\
\hline$C(20)$ & $3008(1)$ & $3904(1)$ & $6802(1)$ & $20(1)$ \\
\hline$C(21)$ & $3416(1)$ & $2147(1)$ & $5757(1)$ & $17(1)$ \\
\hline$C(22)$ & $3433(1)$ & $1430(1)$ & $5229(1)$ & 21(1) \\
\hline$C(23)$ & $3466(1)$ & $340(1)$ & $5351(1)$ & $26(1)$ \\
\hline$C(24)$ & $3485(1)$ & $-43(1)$ & $5997(1)$ & $28(1)$ \\
\hline$C(25)$ & 3483(1) & $665(2)$ & $6523(1)$ & $35(1)$ \\
\hline$C(26)$ & $3449(1)$ & $1754(1)$ & $6406(1)$ & $29(1)$ \\
\hline$C(27)$ & 3413(1) & 6402(1) & $6065(1)$ & $16(1)$ \\
\hline$C(28)$ & $3349(1)$ & 6903(1) & $5445(1)$ & $21(1)$ \\
\hline$C(29)$ & $3039(1)$ & $7554(1)$ & $5317(1)$ & $28(1)$ \\
\hline$C(30)$ & $2796(1)$ & $7725(1)$ & $5809(1)$ & $30(1)$ \\
\hline$C(31)$ & $2860(1)$ & $7242(1)$ & $6426(1)$ & $27(1)$ \\
\hline$C(32)$ & $3167(1)$ & $6579(1)$ & $6557(1)$ & $20(1)$ \\
\hline$C(33)$ & $3896(1)$ & $5298(1)$ & 7072(1) & $15(1)$ \\
\hline$C(34)$ & $3906(1)$ & $6152(1)$ & $7523(1)$ & $18(1)$ \\
\hline$C(35)$ & $3995(1)$ & $5971(1)$ & $8198(1)$ & $23(1)$ \\
\hline$C(36)$ & 4073(1) & 4947(1) & $8426(1)$ & $24(1)$ \\
\hline$C(37)$ & $4063(1)$ & 4096(1) & 7983(1) & $20(1)$ \\
\hline$C(38)$ & $3978(1)$ & $4270(1)$ & $7307(1)$ & $16(1)$ \\
\hline$C(39)$ & $4263(1)$ & 7798(1) & $6095(1)$ & $26(1)$ \\
\hline $\mathrm{C}(40)$ & $4540(1)$ & $7256(2)$ & $6487(1)$ & $28(1)$ \\
\hline $\mathrm{C}(41 \mathrm{~A})$ & $4944(2)$ & 7604(4) & $6449(3)$ & $24(1)$ \\
\hline $\mathrm{C}(42 \mathrm{~A})$ & 5144(1) & 6881(4) & $5966(3)$ & $25(1)$ \\
\hline$C(41 B)$ & 4978(3) & $7189(8)$ & $6516(5)$ & $34(2)$ \\
\hline$C(42 B)$ & $5121(2)$ & $7276(7)$ & $5828(4)$ & $29(1)$ \\
\hline$C(43)$ & $4890(1)$ & 6604(1) & $5339(1)$ & $24(1)$ \\
\hline$C(44)$ & $4630(1)$ & $7172(1)$ & 4963(1) & $28(1)$ \\
\hline$C(45 A)$ & $4610(1)$ & $8385(3)$ & $5101(2)$ & $22(1)$ \\
\hline$C(46 A)$ & $4279(1)$ & $8616(4)$ & $5521(3)$ & $23(1)$ \\
\hline$C(45 B)$ & $4449(2)$ & $8280(5)$ & $4967(4)$ & $34(1)$ \\
\hline $\mathrm{C}(46 \mathrm{~B})$ & $4415(2)$ & $8657(6)$ & $5668(5)$ & $34(2)$ \\
\hline $\mathrm{F}(1 \mathrm{~A})$ & $3643(3)$ & $8195(8)$ & $3015(4)$ & $38(2)$ \\
\hline $\mathrm{F}(2 \mathrm{~A})$ & 3394(3) & $8882(5)$ & $3922(3)$ & $30(1)$ \\
\hline $\mathrm{F}(3 \mathrm{~A})$ & $3198(2)$ & $7293(6)$ & $3491(4)$ & $90(2)$ \\
\hline $\mathrm{F}(4 \mathrm{~A})$ & $3764(2)$ & $7460(5)$ & $4037(2)$ & $70(2)$ \\
\hline $\mathrm{B}(1 \mathrm{~A})$ & $3494(2)$ & 7951(5) & $3616(4)$ & $32(2)$ \\
\hline $\mathrm{F}(1 \mathrm{~B})$ & $3676(4)$ & $8207(9)$ & $3055(6)$ & $31(2)$ \\
\hline $\mathrm{F}(2 \mathrm{~B})$ & $3370(4)$ & $8815(9)$ & $3911(7)$ & $50(3)$ \\
\hline $\mathrm{F}(3 \mathrm{~B})$ & $3118(3)$ & $7517(10)$ & $3267(5)$ & $88(3)$ \\
\hline $\mathrm{F}(4 \mathrm{~B})$ & $3618(2)$ & $7151(5)$ & $3954(4)$ & $52(2)$ \\
\hline $\mathrm{B}(1 \mathrm{~B})$ & $3446(3)$ & $7916(7)$ & $3550(5)$ & $29(2)$ \\
\hline Cl11 & 4171(1) & $712(1)$ & $4062(1)$ & $34(1)$ \\
\hline
\end{tabular}




\begin{tabular}{lllll}
$\mathrm{C} 121$ & $3505(1)$ & $1456(1)$ & $3312(1)$ & $37(1)$ \\
$\mathrm{C} 131$ & $4165(1)$ & $972(1)$ & $2635(1)$ & $34(1)$ \\
$\mathrm{C} 11$ & $3899(1)$ & $618(1)$ & $3302(1)$ & $26(1)$ \\
\hline
\end{tabular}

Table S20. Anisotropic displacement parameters $\left(\AA^{2} \mathrm{x} 10^{3}\right)$ for $[\mathbf{4 a}]\left[\mathrm{BF}_{4}\right]$. The anisotropic displacement factor exponent takes the form: $-2 \pi^{2}\left[\mathrm{~h}^{2} \mathrm{a} * 2 \mathrm{U}^{11}+\ldots+2 \mathrm{~h} \mathrm{k} \mathrm{a}^{*} \mathrm{~b}^{*} \mathrm{U}^{12}\right]$

\begin{tabular}{|c|c|c|c|c|c|c|}
\hline & $\mathrm{U}^{11}$ & $\mathrm{U}^{22}$ & $\mathrm{U}^{33}$ & $\mathrm{U}^{23}$ & $\mathrm{U}^{13}$ & $\mathrm{U}^{12}$ \\
\hline$\overline{R h}(1)$ & $13(1)$ & $14(1)$ & $13(1)$ & $-1(1)$ & $2(1)$ & $-2(1)$ \\
\hline $\mathrm{S}(1)$ & $13(1)$ & $14(1)$ & $12(1)$ & $-2(1)$ & 1(1) & $-1(1)$ \\
\hline $\mathrm{P}(1)$ & $13(1)$ & $14(1)$ & $13(1)$ & $-1(1)$ & $0(1)$ & $-2(1)$ \\
\hline $\mathrm{P}(2)$ & $12(1)$ & $13(1)$ & $12(1)$ & $-1(1)$ & 1(1) & $-1(1)$ \\
\hline $\mathrm{O}(1)$ & $18(1)$ & $15(1)$ & $14(1)$ & $-2(1)$ & $4(1)$ & $-2(1)$ \\
\hline $\mathrm{O}(2)$ & $19(1)$ & $19(1)$ & $15(1)$ & $-5(1)$ & 1(1) & $-1(1)$ \\
\hline $\mathrm{C}(1)$ & $13(1)$ & $15(1)$ & $14(1)$ & $-3(1)$ & 1(1) & $-1(1)$ \\
\hline$C(2)$ & $14(1)$ & $18(1)$ & $15(1)$ & 1(1) & $4(1)$ & $2(1)$ \\
\hline$C(3)$ & $18(1)$ & $19(1)$ & $18(1)$ & $-2(1)$ & $3(1)$ & $0(1)$ \\
\hline$C(4)$ & $17(1)$ & $26(1)$ & $18(1)$ & $0(1)$ & 1(1) & 1(1) \\
\hline$C(5)$ & $21(1)$ & $28(1)$ & $22(1)$ & $5(1)$ & $4(1)$ & $6(1)$ \\
\hline$C(6)$ & $28(1)$ & $17(1)$ & $32(1)$ & $2(1)$ & $4(1)$ & $4(1)$ \\
\hline$C(7)$ & $21(1)$ & $18(1)$ & $25(1)$ & $-2(1)$ & $2(1)$ & $0(1)$ \\
\hline $\mathrm{C}(8)$ & $28(1)$ & $33(1)$ & $34(1)$ & $6(1)$ & $0(1)$ & 11(1) \\
\hline $\mathrm{C}(9)$ & $16(1)$ & $18(1)$ & $15(1)$ & $-1(1)$ & $-1(1)$ & $-1(1)$ \\
\hline$C(10)$ & $18(1)$ & $18(1)$ & $26(1)$ & $2(1)$ & $-4(1)$ & $-5(1)$ \\
\hline $\mathrm{C}(11)$ & $29(1)$ & $26(1)$ & $25(1)$ & $9(1)$ & $-6(1)$ & $-8(1)$ \\
\hline$C(12)$ & $30(1)$ & $29(1)$ & $21(1)$ & $4(1)$ & $-8(1)$ & $-6(1)$ \\
\hline$C(13)$ & $23(1)$ & $25(1)$ & $24(1)$ & $-1(1)$ & $-6(1)$ & $-7(1)$ \\
\hline$C(14)$ & $19(1)$ & $19(1)$ & $20(1)$ & 1(1) & $0(1)$ & $-5(1)$ \\
\hline$C(15)$ & $14(1)$ & $16(1)$ & $22(1)$ & $-5(1)$ & $3(1)$ & $-4(1)$ \\
\hline$C(16)$ & $18(1)$ & $21(1)$ & $27(1)$ & $-5(1)$ & $0(1)$ & $-1(1)$ \\
\hline$C(17)$ & $16(1)$ & $28(1)$ & $42(1)$ & $-11(1)$ & $0(1)$ & $2(1)$ \\
\hline$C(18)$ & $17(1)$ & $34(1)$ & $40(1)$ & $-18(1)$ & $10(1)$ & $-6(1)$ \\
\hline C(19) & $24(1)$ & $31(1)$ & $25(1)$ & $-11(1)$ & $7(1)$ & $-10(1)$ \\
\hline$C(20)$ & $17(1)$ & $22(1)$ & $22(1)$ & $-5(1)$ & $3(1)$ & $-5(1)$ \\
\hline$C(21)$ & $20(1)$ & $13(1)$ & $18(1)$ & $-1(1)$ & 1(1) & $-2(1)$ \\
\hline$C(22)$ & $24(1)$ & $20(1)$ & $20(1)$ & $-2(1)$ & 1(1) & $0(1)$ \\
\hline$C(23)$ & $29(1)$ & $19(1)$ & $29(1)$ & $-6(1)$ & $-1(1)$ & 1(1) \\
\hline$C(24)$ & $34(1)$ & $16(1)$ & $36(1)$ & $2(1)$ & $-1(1)$ & $2(1)$ \\
\hline$C(25)$ & $55(1)$ & $25(1)$ & $25(1)$ & $6(1)$ & 2(1) & $7(1)$ \\
\hline$C(26)$ & $47(1)$ & $21(1)$ & $20(1)$ & $-2(1)$ & $0(1)$ & $5(1)$ \\
\hline$C(27)$ & $15(1)$ & $12(1)$ & $21(1)$ & $-2(1)$ & $-1(1)$ & $-1(1)$ \\
\hline $\mathrm{C}(28)$ & $20(1)$ & $18(1)$ & $24(1)$ & $0(1)$ & $-1(1)$ & $-2(1)$ \\
\hline C(29) & $27(1)$ & $21(1)$ & $33(1)$ & $4(1)$ & $-9(1)$ & 1(1) \\
\hline$C(30)$ & $20(1)$ & $21(1)$ & $48(1)$ & $-4(1)$ & $-7(1)$ & $6(1)$ \\
\hline$C(31)$ & $18(1)$ & $23(1)$ & $40(1)$ & $-8(1)$ & $5(1)$ & $2(1)$ \\
\hline$C(32)$ & $17(1)$ & $18(1)$ & $26(1)$ & $-2(1)$ & $2(1)$ & $0(1)$ \\
\hline C(33) & $12(1)$ & $18(1)$ & $14(1)$ & $-1(1)$ & 1(1) & $-2(1)$ \\
\hline$C(34)$ & $19(1)$ & $18(1)$ & $17(1)$ & $-3(1)$ & $2(1)$ & $-2(1)$ \\
\hline$C(35)$ & $24(1)$ & $28(1)$ & $17(1)$ & $-6(1)$ & 1(1) & $0(1)$ \\
\hline$C(36)$ & $24(1)$ & $34(1)$ & $13(1)$ & 1(1) & 1(1) & 1(1) \\
\hline$C(37)$ & $18(1)$ & $24(1)$ & $19(1)$ & $5(1)$ & $2(1)$ & 1(1) \\
\hline C(38) & $14(1)$ & $18(1)$ & $18(1)$ & $-2(1)$ & $2(1)$ & $-1(1)$ \\
\hline C(39) & $32(1)$ & $16(1)$ & $32(1)$ & $-9(1)$ & $15(1)$ & $-4(1)$ \\
\hline
\end{tabular}




\begin{tabular}{lllllll}
$\mathrm{C}(40)$ & $23(1)$ & $39(1)$ & $21(1)$ & $-15(1)$ & $6(1)$ & $-13(1)$ \\
$\mathrm{C}(41 \mathrm{~A})$ & $17(2)$ & $32(3)$ & $23(2)$ & $-7(2)$ & $0(1)$ & $-7(2)$ \\
$\mathrm{C}(42 \mathrm{~A})$ & $15(1)$ & $25(2)$ & $34(2)$ & $1(2)$ & $3(1)$ & $-4(2)$ \\
$\mathrm{C}(41 \mathrm{~B})$ & $23(3)$ & $46(5)$ & $33(3)$ & $-13(4)$ & $4(2)$ & $-14(3)$ \\
$\mathrm{C}(42 \mathrm{~B})$ & $19(2)$ & $34(4)$ & $34(3)$ & $-6(3)$ & $7(2)$ & $-11(3)$ \\
$\mathrm{C}(43)$ & $18(1)$ & $27(1)$ & $29(1)$ & $-7(1)$ & $13(1)$ & $-8(1)$ \\
$\mathrm{C}(44)$ & $40(1)$ & $22(1)$ & $24(1)$ & $4(1)$ & $19(1)$ & $-2(1)$ \\
$\mathrm{C}(45 \mathrm{~A})$ & $23(2)$ & $17(1)$ & $28(2)$ & $6(1)$ & $7(2)$ & $-1(2)$ \\
$\mathrm{C}(46 \mathrm{~A})$ & $22(2)$ & $14(1)$ & $35(2)$ & $1(1)$ & $6(2)$ & $1(2)$ \\
$\mathrm{C}(45 \mathrm{~B})$ & $35(3)$ & $25(2)$ & $44(3)$ & $12(2)$ & $9(3)$ & $-2(3)$ \\
$\mathrm{C}(46 \mathrm{~B})$ & $31(4)$ & $16(2)$ & $58(4)$ & $-1(2)$ & $14(3)$ & $-2(3)$ \\
$\mathrm{F}(1 \mathrm{~A})$ & $63(4)$ & $35(3)$ & $18(2)$ & $3(2)$ & $5(2)$ & $6(2)$ \\
$\mathrm{F}(2 \mathrm{~A})$ & $35(3)$ & $21(2)$ & $37(3)$ & $-5(2)$ & $12(2)$ & $1(2)$ \\
$\mathrm{F}(3 \mathrm{~A})$ & $111(5)$ & $57(3)$ & $105(5)$ & $-27(3)$ & $37(3)$ & $-61(3)$ \\
$\mathrm{F}(4 \mathrm{~A})$ & $105(4)$ & $81(4)$ & $26(1)$ & $7(2)$ & $9(2)$ & $64(3)$ \\
$\mathrm{B}(1 \mathrm{~A})$ & $44(3)$ & $20(3)$ & $32(3)$ & $2(2)$ & $7(2)$ & $1(2)$ \\
$\mathrm{F}(1 \mathrm{~B})$ & $40(3)$ & $15(3)$ & $39(5)$ & $5(3)$ & $12(3)$ & $2(2)$ \\
$\mathrm{F}(2 \mathrm{~B})$ & $45(5)$ & $35(4)$ & $72(6)$ & $-12(4)$ & $17(4)$ & $10(4)$ \\
$\mathrm{F}(3 \mathrm{~B})$ & $59(3)$ & $108(7)$ & $94(6)$ & $-32(4)$ & $-4(3)$ & $-48(3)$ \\
$\mathrm{F}(4 \mathrm{~B})$ & $97(5)$ & $33(2)$ & $32(3)$ & $14(2)$ & $35(3)$ & $26(2)$ \\
$\mathrm{B}(1 \mathrm{~B})$ & $38(4)$ & $18(4)$ & $33(4)$ & $-5(3)$ & $12(3)$ & $0(3)$ \\
$\mathrm{C} 111$ & $37(1)$ & $35(1)$ & $29(1)$ & $-2(1)$ & $-2(1)$ & $1(1)$ \\
$\mathrm{C} 121$ & $24(1)$ & $37(1)$ & $49(1)$ & $10(1)$ & $5(1)$ & $-1(1)$ \\
$\mathrm{C} 131$ & $38(1)$ & $30(1)$ & $34(1)$ & $11(1)$ & $9(1)$ & $5(1)$ \\
$\mathrm{C} 11$ & $30(1)$ & $20(1)$ & $29(1)$ & $4(1)$ & $0(1)$ & $-4(1)$ \\
\hline
\end{tabular}




\subsection{Crystal Structure Determination of [PPN][4b]}

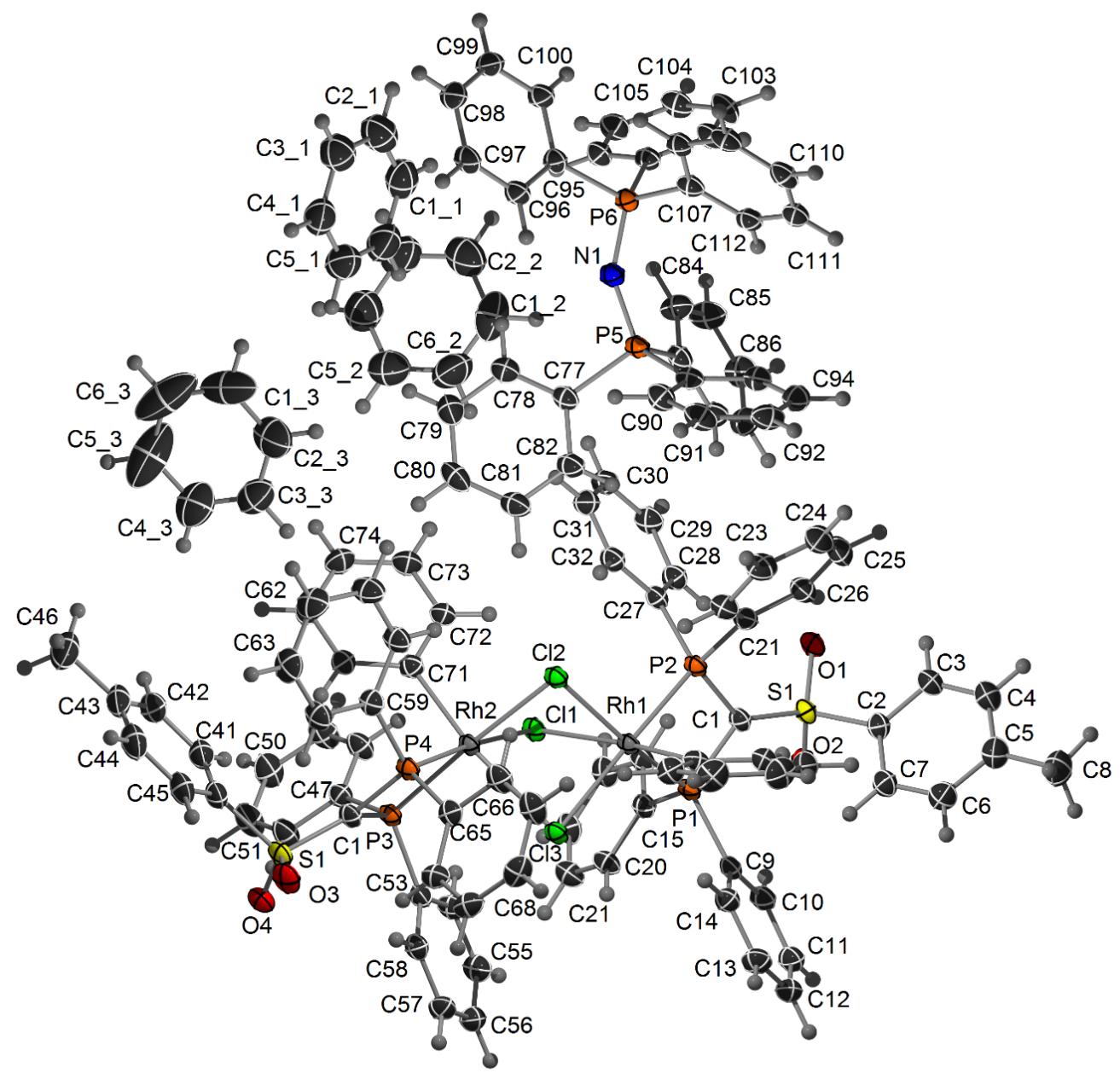

Figure S27. ORTEP Plot of [PPN][4b]. Ellipsoids are drawn at the 50\% probability level.

Table S21. Atomic coordinates ( $\left.\times 10^{4}\right)$ and equivalent isotropic displacement parameters $\left(\AA^{2} \times 10^{3}\right)$ for $[\mathrm{PPN}][\mathbf{4 b}] . \mathrm{U}(\mathrm{eq})$ is defined as one third of the trace of the orthogonalized $\mathrm{U}^{\mathrm{ij}}$ tensor.

\begin{tabular}{lllll}
\hline & $\mathrm{x}$ & $\mathrm{y}$ & $\mathrm{z}$ & $\mathrm{U}(\mathrm{eq})$ \\
\hline $\mathrm{Rh}(1)$ & $4500(1)$ & $1339(1)$ & $3167(1)$ & $20(1)$ \\
$\mathrm{Rh}(2)$ & $5925(1)$ & $1932(1)$ & $4004(1)$ & $20(1)$ \\
$\mathrm{Cl}(1)$ & $4526(1)$ & $1593(1)$ & $4216(1)$ & $23(1)$ \\
$\mathrm{Cl}(2)$ & $5059(1)$ & $2064(1)$ & $3019(1)$ & $23(1)$ \\
$\mathrm{Cl}(3)$ & $6102(1)$ & $1218(1)$ & $3539(1)$ & $23(1)$ \\
$\mathrm{S}(1)$ & $1768(1)$ & $612(1)$ & $2910(1)$ & $24(1)$ \\
$\mathrm{S}(2)$ & $8840(1)$ & $2181(1)$ & $4937(1)$ & $27(1)$ \\
$\mathrm{P}(1)$ & $3797(1)$ & $747(1)$ & $3384(1)$ & $21(1)$ \\
$\mathrm{P}(2)$ & $3016(1)$ & $1418(1)$ & $2858(1)$ & $21(1)$ \\
$\mathrm{P}(3)$ & $6887(1)$ & $1798(1)$ & $4828(1)$ & $21(1)$ \\
$\mathrm{P}(4)$ & $7277(1)$ & $2197(1)$ & $3865(1)$ & $22(1)$ \\
$\mathrm{P}(5)$ & $1784(1)$ & $3463(1)$ & $1773(1)$ & $23(1)$ \\
$\mathrm{P}(6)$ & $800(1)$ & $4274(1)$ & $1416(1)$ & $23(1)$ \\
$\mathrm{O}(1)$ & $975(1)$ & $879(1)$ & $2889(1)$ & $28(1)$
\end{tabular}




\begin{tabular}{|c|c|c|c|c|}
\hline $\mathrm{O}(2)$ & $1845(1)$ & $272(1)$ & $3329(1)$ & $29(1)$ \\
\hline $\mathrm{O}(3)$ & $9504(1)$ & $2164(1)$ & $4521(1)$ & $35(1)$ \\
\hline $\mathrm{O}(4)$ & 8998(1) & 1921(1) & $5461(1)$ & $32(1)$ \\
\hline $\mathrm{N}(1)$ & $1460(1)$ & $3930(1)$ & $1721(1)$ & $26(1)$ \\
\hline$C(1)$ & 2732(1) & $898(1)$ & $3015(1)$ & $23(1)$ \\
\hline$C(2)$ & $1678(2)$ & $374(1)$ & 2192(1) & $26(1)$ \\
\hline$C(3)$ & $879(2)$ & $406(1)$ & $1816(1)$ & $33(1)$ \\
\hline C(4) & 797(2) & 198(1) & $1276(1)$ & $39(1)$ \\
\hline $\mathrm{C}(5)$ & $1495(2)$ & $-39(1)$ & $1103(1)$ & $37(1)$ \\
\hline C(6) & 2294(2) & $-64(1)$ & $1488(1)$ & $34(1$ \\
\hline$C(7)$ & $2389(2)$ & $136(1)$ & $2030(1)$ & $30(1)$ \\
\hline $\mathrm{C}(8)$ & $1407(2)$ & $-268(1)$ & $517(1)$ & $50(1$ \\
\hline $\mathrm{C}(9)$ & $4237(1)$ & $237(1)$ & $3195(1)$ & $24(1)$ \\
\hline$C(10)$ & $3844(2)$ & $-125(1)$ & $3388(1)$ & $28(1$ \\
\hline $\mathrm{C}(11)$ & $4194(2)$ & $-514(1)$ & $3277(1)$ & $32(1$ \\
\hline$C(12)$ & $4939(2)$ & $-547(1)$ & $2967(1)$ & $33(1$ \\
\hline $\mathrm{C}(13)$ & $5337(2)$ & $-189(1)$ & $2778(1)$ & $33(1$ \\
\hline$C(14)$ & 4995(2) & 203(1) & 2893(1) & $27(1$ \\
\hline$C(15)$ & $3726(2)$ & $680(1)$ & 4171(1) & $24(1$ \\
\hline$C(16)$ & $2967(2)$ & $816(1)$ & $4418(1)$ & $28(1$ \\
\hline$C(17)$ & 2931(2) & 785(1) & $5023(1)$ & $31(1)$ \\
\hline$C(18)$ & $3657(2)$ & $618(1)$ & $5388(1)$ & $32(1$ \\
\hline$C(19)$ & $4418(2)$ & $485(1)$ & $5148(1)$ & $32(1$ \\
\hline $\mathrm{C}(20)$ & $4458(2)$ & $517(1)$ & $4542(1)$ & $28(1$ \\
\hline$C(21)$ & $2663(2)$ & 1587(1) & 2098(1) & $26(1$ \\
\hline$C(22)$ & $3215(2)$ & 1843(1) & $1796(1)$ & $30(1$ \\
\hline$C(23)$ & 2899(2) & 1991(1) & $1236(1)$ & $37(1$ \\
\hline $\mathrm{C}(24)$ & $2041(2)$ & $1882(1)$ & $972(1)$ & $42(1$ \\
\hline$C(25)$ & $1496(2)$ & $1631(1)$ & 1271(1) & $42(1)$ \\
\hline$C(26)$ & $1799(2)$ & $1487(1)$ & 1834(1) & $33(1)$ \\
\hline$C(27)$ & $2420(1)$ & 1813(1) & $3237(1)$ & $24(1)$ \\
\hline$C(28)$ & $1637(2)$ & 1731(1) & $3493(1)$ & $28(1)$ \\
\hline$C(29)$ & $1155(2)$ & 2058(1) & $3706(1)$ & $31(1)$ \\
\hline$C(30)$ & $1451(2)$ & $2466(1)$ & $3676(1)$ & $31(1)$ \\
\hline$C(31)$ & $2243(2)$ & 2551(1) & $3431(1)$ & $30(1)$ \\
\hline$C(32)$ & 2718(2) & $2226(1)$ & $3210(1)$ & $27(1)$ \\
\hline$C(33)$ & $4667(2)$ & 1158(1) & $2321(1)$ & $24(1)$ \\
\hline$C(34)$ & $4073(2)$ & 913(1) & 1944(1) & $27(1)$ \\
\hline$C(35)$ & $4205(2)$ & $839(1)$ & $1362(1)$ & $33(1)$ \\
\hline$C(36)$ & 4941(2) & 1010(1) & $1134(1)$ & $39(1)$ \\
\hline$C(37)$ & $5550(2)$ & $1248(1)$ & $1504(1)$ & $37(1)$ \\
\hline$C(38)$ & $5427(2)$ & 1318(1) & 2091(1) & $30(1)$ \\
\hline$C(39)$ & 7792(1) & 2081(1) & $4587(1)$ & $24(1)$ \\
\hline $\mathrm{C}(40)$ & $8885(2)$ & $2710(1)$ & $5183(1)$ & $31(1)$ \\
\hline $\mathrm{C}(41)$ & $8262(2)$ & 2861(1) & $5534(1)$ & $34(1)$ \\
\hline $\mathrm{C}(42)$ & $8296(2)$ & $3277(1)$ & $5696(1)$ & $39(1)$ \\
\hline $\mathrm{C}(43)$ & $8958(2)$ & $3543(1)$ & $5526(1)$ & $42(1)$ \\
\hline $\mathrm{C}(44)$ & $9577(2)$ & $3384(1)$ & $5179(1)$ & $43(1)$ \\
\hline$C(45)$ & $9551(2)$ & 2971(1) & $5008(1)$ & $38(1)$ \\
\hline $\mathrm{C}(46)$ & $9004(3)$ & $3994(1)$ & $5723(2)$ & $59(1)$ \\
\hline$C(47)$ & $6561(2)$ & 1929(1) & $5557(1)$ & $25(1)$ \\
\hline$C(48)$ & $5668(2)$ & 2027(1) & $5618(1)$ & $31(1)$ \\
\hline C(49) & $5415(2)$ & 2107(1) & $6174(1)$ & $38(1)$ \\
\hline$C(50)$ & $6049(2)$ & 2094(1) & $6671(1)$ & $42(1)$ \\
\hline$C(51)$ & $6940(2)$ & 1991(1) & $6612(1)$ & $42(1)$ \\
\hline
\end{tabular}




\begin{tabular}{|c|c|c|c|c|}
\hline$C(52)$ & $7195(2)$ & $1907(1)$ & $6061(1)$ & $34(1)$ \\
\hline $\mathrm{C}(53)$ & $7186(2)$ & $1250(1)$ & 4918(1) & $24(1)$ \\
\hline$C(54)$ & $6576(2)$ & $972(1)$ & 5133(1) & $28(1)$ \\
\hline$C(55)$ & $6785(2)$ & $552(1)$ & $5178(1)$ & $33(1)$ \\
\hline$C(56)$ & $7592(2)$ & 401(1) & $5017(1)$ & $32(1)$ \\
\hline$C(57)$ & $8192(2)$ & $675(1)$ & 4804(1) & $30(1)$ \\
\hline$C(58)$ & $7987(2)$ & 1097(1) & $4752(1)$ & $26(1)$ \\
\hline $\mathrm{C}(59)$ & $7361(2)$ & 2744(1) & $3658(1)$ & $26(1)$ \\
\hline$C(60)$ & $6614(2)$ & $2965(1)$ & $3398(1)$ & $31(1)$ \\
\hline $\mathrm{C}(61)$ & $6702(2)$ & $3381(1)$ & $3247(1)$ & $39(1)$ \\
\hline$C(62)$ & $7529(2)$ & $3582(1)$ & $3372(1)$ & $40(1)$ \\
\hline$C(63)$ & $8274(2)$ & $3362(1)$ & $3620(1)$ & $40(1)$ \\
\hline$C(64)$ & $8200(2)$ & 2941(1) & $3750(1)$ & $33(1)$ \\
\hline$C(65)$ & $7809(2)$ & 1951(1) & $3270(1)$ & $26(1)$ \\
\hline$C(66)$ & $7388(2)$ & $2015(1)$ & 2693(1) & $32(1)$ \\
\hline $\mathrm{C}(67)$ & $7760(2)$ & $1850(1)$ & $2216(1)$ & $36(1)$ \\
\hline $\mathrm{C}(68)$ & $8564(2)$ & $1625(1)$ & $2305(1)$ & $34(1)$ \\
\hline $\mathrm{C}(69)$ & $8990(2)$ & $1566(1)$ & $2876(1)$ & $32(1)$ \\
\hline$C(70)$ & $8610(2)$ & $1725(1)$ & $3359(1)$ & $28(1)$ \\
\hline $\mathrm{C}(71)$ & $5537(2)$ & $2482(1)$ & $4362(1)$ & $25(1)$ \\
\hline$C(72)$ & $4609(2)$ & 2571(1) & $4277(1)$ & $28(1)$ \\
\hline$C(73)$ & $4249(2)$ & $2898(1)$ & 4571(1) & $36(1)$ \\
\hline$C(74)$ & $4801(2)$ & $3152(1)$ & 4948(1) & $39(1)$ \\
\hline$C(75)$ & $5726(2)$ & $3082(1)$ & $5019(1)$ & $36(1)$ \\
\hline$C(76)$ & $6090(2)$ & $2755(1)$ & $4726(1)$ & $28(1)$ \\
\hline $\mathrm{C}(77)$ & $2764(2)$ & $3455(1)$ & $2324(1)$ & $26(1)$ \\
\hline$C(78)$ & $2897(2)$ & $3778(1)$ & $2729(1)$ & $37(1)$ \\
\hline$C(79)$ & $3611(2)$ & $3759(1)$ & 3181(1) & $44(1)$ \\
\hline $\mathrm{C}(80)$ & $4196(2)$ & $3421(1)$ & $3223(1)$ & $39(1)$ \\
\hline $\mathrm{C}(81)$ & $4076(2)$ & $3105(1)$ & 2811(1) & $35(1)$ \\
\hline $\mathrm{C}(82)$ & $3359(2)$ & $3117(1)$ & $2362(1)$ & $31(1)$ \\
\hline $\mathrm{C}(83)$ & $956(2)$ & $3117(1)$ & 2023(1) & $26(1)$ \\
\hline$C(84)$ & 214(2) & $3285(1)$ & $2243(1)$ & $35(1)$ \\
\hline$C(85)$ & $-426(2)$ & $3025(1)$ & 2451(1) & $41(1)$ \\
\hline$C(86)$ & $-320(2)$ & 2601(1) & $2445(1)$ & $38(1)$ \\
\hline $\mathrm{C}(87)$ & $426(2)$ & $2427(1)$ & $2220(1)$ & $34(1)$ \\
\hline $\mathrm{C}(88)$ & $1065(2)$ & 2682(1) & 2011(1) & $30(1)$ \\
\hline $\mathrm{C}(89)$ & $2124(2)$ & $3244(1)$ & $1108(1)$ & $26(1)$ \\
\hline $\mathrm{C}(90)$ & $2955(2)$ & $3361(1)$ & $939(1)$ & $32(1)$ \\
\hline $\mathrm{C}(91)$ & $3209(2)$ & $3226(1)$ & $406(1)$ & $36(1)$ \\
\hline $\mathrm{C}(92)$ & $2638(2)$ & 2978(1) & $33(1)$ & $36(1)$ \\
\hline $\mathrm{C}(93)$ & $1804(2)$ & $2859(1)$ & 196(1) & $33(1)$ \\
\hline $\mathrm{C}(94)$ & $1549(2)$ & 2993(1) & 731(1) & $29(1)$ \\
\hline $\mathrm{C}(95)$ & $1270(2)$ & $4780(1)$ & 1584(1) & $26(1)$ \\
\hline $\mathrm{C}(96)$ & $2197(2)$ & $4843(1)$ & 1573(1) & $29(1)$ \\
\hline $\mathrm{C}(97)$ & $2546(2)$ & $5242(1)$ & 1620(1) & $33(1)$ \\
\hline $\mathrm{C}(98)$ & 1989(2) & $5579(1)$ & 1689(1) & $37(1)$ \\
\hline $\mathrm{C}(99)$ & $1075(2)$ & $5517(1)$ & 1713(1) & $38(1)$ \\
\hline$C(100)$ & 708(2) & $5120(1)$ & $1655(1)$ & $31(1)$ \\
\hline$C(101)$ & $-281(2)$ & $4263(1)$ & 1687(1) & $25(1)$ \\
\hline$C(102)$ & $-1056(2)$ & 4133(1) & 1341(1) & $30(1)$ \\
\hline$C(103)$ & $-1863(2)$ & 4104(1) & 1587(1) & $35(1)$ \\
\hline$C(104)$ & $-1886(2)$ & $4202(1)$ & 2173(1) & $35(1)$ \\
\hline$C(105)$ & $-1110(2)$ & $4333(1)$ & $2524(1)$ & $32(1)$ \\
\hline$C(106)$ & $-308(2)$ & $4364(1)$ & $2283(1)$ & $29(1)$ \\
\hline
\end{tabular}




\begin{tabular}{lllll}
$\mathrm{C}(107)$ & $635(1)$ & $4237(1)$ & $619(1)$ & $25(1)$ \\
$\mathrm{C}(108)$ & $778(2)$ & $4582(1)$ & $266(1)$ & $27(1)$ \\
$\mathrm{C}(109)$ & $738(2)$ & $4536(1)$ & $-341(1)$ & $31(1)$ \\
$\mathrm{C}(110)$ & $560(2)$ & $4150(1)$ & $-600(1)$ & $32(1)$ \\
$\mathrm{C}(111)$ & $393(2)$ & $3809(1)$ & $-254(1)$ & $31(1)$ \\
$\mathrm{C}(112)$ & $424(2)$ & $3852(1)$ & $354(1)$ & $28(1)$ \\
$\mathrm{C} 11$ & $1642(2)$ & $4980(1)$ & $3383(2)$ & $60(1)$ \\
$\mathrm{C} 21$ & $1778(3)$ & $5370(1)$ & $3152(2)$ & $63(1)$ \\
$\mathrm{C} 31$ & $2644(3)$ & $5515(1)$ & $3123(2)$ & $59(1)$ \\
$\mathrm{C} 41$ & $3364(2)$ & $5268(1)$ & $3320(2)$ & $56(1)$ \\
$\mathrm{C} 51$ & $3230(3)$ & $4877(1)$ & $3543(2)$ & $62(1)$ \\
$\mathrm{C} 61$ & $2359(3)$ & $4735(1)$ & $3585(2)$ & $65(1)$ \\
$\mathrm{C} 12$ & $1115(3)$ & $3733(1)$ & $3914(2)$ & $71(1)$ \\
$\mathrm{C} 22$ & $647(3)$ & $4080(1)$ & $4104(2)$ & $71(1)$ \\
$\mathrm{C} 32$ & $987(3)$ & $4271(1)$ & $4630(2)$ & $72(1)$ \\
$\mathrm{C} 42$ & $1757(3)$ & $4131(1)$ & $4953(2)$ & $72(1)$ \\
$\mathrm{C} 52$ & $2202(3)$ & $3797(1)$ & $4768(2)$ & $67(1)$ \\
$\mathrm{C} 62$ & $1884(3)$ & $3600(1)$ & $4259(2)$ & $71(1)$ \\
$\mathrm{C} 13$ & $5611(3)$ & $4760(1)$ & $4123(3)$ & $96(2)$ \\
$\mathrm{C} 23$ & $5668(3)$ & $4352(1)$ & $3963(2)$ & $73(1)$ \\
$\mathrm{C} 33$ & $6090(3)$ & $4065(1)$ & $4352(2)$ & $60(1)$ \\
$\mathrm{C} 43$ & $6453(3)$ & $4191(1)$ & $4893(2)$ & $71(1)$ \\
$\mathrm{C} 53$ & $6411(5)$ & $4599(2)$ & $5061(3)$ & $107(2)$ \\
$\mathrm{C} 63$ & $6000(5)$ & $4888(2)$ & $4676(4)$ & $112(2)$ \\
\hline
\end{tabular}

Table S22. Anisotropic displacement parameters $\left(\AA^{2} \mathrm{x} 10^{3}\right)$ for $[\mathrm{PPN}][\mathbf{4 b}]$. The anisotropic displacement factor exponent takes the form: $-2 \pi^{2}\left[h^{2} a^{* 2} U^{11}+\ldots+2 h k a^{*} b^{*} U^{12}\right]$

\begin{tabular}{lllllll}
\hline & $\mathrm{U}^{11}$ & $\mathrm{U}^{22}$ & $\mathrm{U}^{33}$ & $\mathrm{U}^{23}$ & $\mathrm{U}^{13}$ & $\mathrm{U}^{12}$ \\
\hline $\mathrm{Rh}(1)$ & $13(1)$ & $22(1)$ & $24(1)$ & $1(1)$ & $2(1)$ & $0(1)$ \\
$\mathrm{Rh}(2)$ & $12(1)$ & $22(1)$ & $24(1)$ & $1(1)$ & $1(1)$ & $0(1)$ \\
$\mathrm{Cl}(1)$ & $15(1)$ & $27(1)$ & $27(1)$ & $0(1)$ & $3(1)$ & $-2(1)$ \\
$\mathrm{Cl}(2)$ & $16(1)$ & $24(1)$ & $27(1)$ & $2(1)$ & $1(1)$ & $0(1)$ \\
$\mathrm{Cl}(3)$ & $15(1)$ & $26(1)$ & $28(1)$ & $1(1)$ & $2(1)$ & $2(1)$ \\
$\mathrm{S}(1)$ & $15(1)$ & $27(1)$ & $29(1)$ & $-1(1)$ & $1(1)$ & $-3(1)$ \\
$\mathrm{S}(2)$ & $15(1)$ & $33(1)$ & $32(1)$ & $-2(1)$ & $0(1)$ & $-1(1)$ \\
$\mathrm{P}(1)$ & $14(1)$ & $23(1)$ & $26(1)$ & $2(1)$ & $2(1)$ & $-1(1)$ \\
$\mathrm{P}(2)$ & $13(1)$ & $24(1)$ & $25(1)$ & $1(1)$ & $1(1)$ & $0(1)$ \\
$\mathrm{P}(3)$ & $14(1)$ & $25(1)$ & $25(1)$ & $0(1)$ & $0(1)$ & $0(1)$ \\
$\mathrm{P}(4)$ & $14(1)$ & $25(1)$ & $27(1)$ & $2(1)$ & $2(1)$ & $-2(1)$ \\
$\mathrm{P}(5)$ & $16(1)$ & $23(1)$ & $29(1)$ & $2(1)$ & $4(1)$ & $0(1)$ \\
$\mathrm{P}(6)$ & $17(1)$ & $22(1)$ & $30(1)$ & $1(1)$ & $4(1)$ & $-1(1)$ \\
$\mathrm{O}(1)$ & $15(1)$ & $34(1)$ & $36(1)$ & $-4(1)$ & $1(1)$ & $0(1)$ \\
$\mathrm{O}(2)$ & $24(1)$ & $30(1)$ & $33(1)$ & $2(1)$ & $3(1)$ & $-5(1)$ \\
$\mathrm{O}(3)$ & $18(1)$ & $46(1)$ & $40(1)$ & $-4(1)$ & $5(1)$ & $-3(1)$ \\
$\mathrm{O}(4)$ & $22(1)$ & $37(1)$ & $34(1)$ & $-1(1)$ & $-4(1)$ & $3(1)$ \\
$\mathrm{N}(1)$ & $21(1)$ & $24(1)$ & $34(1)$ & $2(1)$ & $3(1)$ & $0(1)$ \\
$\mathrm{C}(1)$ & $15(1)$ & $25(1)$ & $29(1)$ & $0(1)$ & $0(1)$ & $-2(1)$ \\
$\mathrm{C}(2)$ & $22(1)$ & $27(1)$ & $30(1)$ & $-1(1)$ & $2(1)$ & $-4(1)$ \\
$\mathrm{C}(3)$ & $24(1)$ & $35(1)$ & $39(1)$ & $-3(1)$ & $-3(1)$ & $2(1)$ \\
$\mathrm{C}(4)$ & $34(1)$ & $43(2)$ & $37(1)$ & $-3(1)$ & $-6(1)$ & $0(1)$ \\
$\mathrm{C}(5)$ & $38(1)$ & $38(1)$ & $35(1)$ & $-4(1)$ & $3(1)$ & $-3(1)$ \\
$\mathrm{C}(6)$ & $29(1)$ & $35(1)$ & $40(1)$ & $-3(1)$ & $9(1)$ & $-2(1)$
\end{tabular}




\begin{tabular}{|c|c|c|c|c|c|c|}
\hline$C(7)$ & $23(1)$ & $31(1)$ & $36(1)$ & $-3(1)$ & $2(1)$ & $-2(1)$ \\
\hline $\mathrm{C}(8)$ & $53(2)$ & $53(2)$ & $43(2)$ & $-15(1)$ & $0(1)$ & $-1(2)$ \\
\hline $\mathrm{C}(9)$ & $16(1)$ & $29(1)$ & $28(1)$ & $1(1)$ & $-2(1)$ & 1(1) \\
\hline $\mathrm{C}(10)$ & $19(1)$ & $29(1)$ & $35(1)$ & $3(1)$ & 1(1) & $-1(1)$ \\
\hline $\mathrm{C}(11)$ & $24(1)$ & $28(1)$ & $42(1)$ & $2(1)$ & $-1(1)$ & $-1(1)$ \\
\hline $\mathrm{C}(12)$ & $27(1)$ & $27(1)$ & $44(1)$ & $-4(1)$ & $-1(1)$ & $5(1)$ \\
\hline $\mathrm{C}(13)$ & $26(1)$ & $34(1)$ & $39(1)$ & $0(1)$ & $7(1)$ & $4(1)$ \\
\hline $\mathrm{C}(14)$ & $22(1)$ & $27(1)$ & $33(1)$ & $-1(1)$ & $3(1)$ & 1(1) \\
\hline $\mathrm{C}(15)$ & $18(1)$ & $25(1)$ & $28(1)$ & 1(1) & 2(1) & $-2(1)$ \\
\hline $\mathrm{C}(16)$ & $18(1)$ & $32(1)$ & $32(1)$ & $0(1)$ & 1(1) & $-2(1)$ \\
\hline $\mathrm{C}(17)$ & $21(1)$ & $39(1)$ & $34(1)$ & $-1(1)$ & $7(1)$ & $-6(1)$ \\
\hline $\mathrm{C}(18)$ & $28(1)$ & $38(1)$ & $29(1)$ & 2(1) & $4(1)$ & $-7(1)$ \\
\hline C(19) & $25(1)$ & $36(1)$ & $33(1)$ & $7(1)$ & $0(1)$ & $2(1)$ \\
\hline $\mathrm{C}(20)$ & $19(1)$ & $33(1)$ & $32(1)$ & $3(1)$ & 2(1) & 1(1) \\
\hline$C(21)$ & $23(1)$ & $26(1)$ & $28(1)$ & 1(1) & 1(1) & $4(1)$ \\
\hline$C(22)$ & $27(1)$ & $32(1)$ & $30(1)$ & $4(1)$ & 1(1) & $3(1)$ \\
\hline$C(23)$ & $45(2)$ & $34(1)$ & $32(1)$ & $8(1)$ & $4(1)$ & $3(1)$ \\
\hline$C(24)$ & $55(2)$ & $36(1)$ & $30(1)$ & $4(1)$ & $-8(1)$ & $5(1)$ \\
\hline$C(25)$ & $42(2)$ & $42(2)$ & $38(2)$ & $2(1)$ & $-15(1)$ & $-2(1)$ \\
\hline$C(26)$ & $29(1)$ & $33(1)$ & $36(1)$ & $4(1)$ & $-5(1)$ & 1(1) \\
\hline$C(27)$ & $18(1)$ & $27(1)$ & $26(1)$ & $0(1)$ & $0(1)$ & 1(1) \\
\hline $\mathrm{C}(28)$ & 21(1) & $28(1)$ & $33(1)$ & $0(1)$ & $4(1)$ & $0(1)$ \\
\hline C(29) & 21(1) & $37(1)$ & $36(1)$ & $-1(1)$ & $8(1)$ & $0(1)$ \\
\hline$C(30)$ & $24(1)$ & $34(1)$ & $34(1)$ & $-1(1)$ & $4(1)$ & $6(1)$ \\
\hline $\mathrm{C}(31)$ & $27(1)$ & $26(1)$ & $36(1)$ & 1(1) & 2(1) & 1(1) \\
\hline$C(32)$ & $20(1)$ & $29(1)$ & $33(1)$ & 1(1) & 2(1) & $1(1)$ \\
\hline$C(33)$ & $23(1)$ & $26(1)$ & $23(1)$ & $2(1)$ & $5(1)$ & $6(1)$ \\
\hline $\mathrm{C}(34)$ & $24(1)$ & $27(1)$ & $29(1)$ & $-1(1)$ & $1(1)$ & 2(1) \\
\hline $\mathrm{C}(35)$ & $35(1)$ & $33(1)$ & $30(1)$ & $-2(1)$ & $0(1)$ & 1(1) \\
\hline $\mathrm{C}(36)$ & $50(2)$ & $40(1)$ & $28(1)$ & $-1(1)$ & $11(1)$ & $3(1)$ \\
\hline $\mathrm{C}(37)$ & $40(1)$ & $38(1)$ & $37(1)$ & $0(1)$ & $17(1)$ & $-3(1)$ \\
\hline $\mathrm{C}(38)$ & $28(1)$ & $30(1)$ & $32(1)$ & $1(1)$ & $8(1)$ & $-2(1)$ \\
\hline $\mathrm{C}(39)$ & $15(1)$ & $27(1)$ & $28(1)$ & $0(1)$ & 1(1) & $-1(1)$ \\
\hline $\mathrm{C}(40)$ & $24(1)$ & $34(1)$ & $32(1)$ & $-2(1)$ & $-4(1)$ & $-4(1)$ \\
\hline $\mathrm{C}(41)$ & $32(1)$ & $36(1)$ & $32(1)$ & $-2(1)$ & $-2(1)$ & $-5(1)$ \\
\hline$C(42)$ & $45(2)$ & $39(1)$ & $31(1)$ & $-2(1)$ & $-3(1)$ & 1(1) \\
\hline$C(43)$ & $55(2)$ & $34(1)$ & $33(1)$ & 1(1) & $-14(1)$ & $-10(1)$ \\
\hline $\mathrm{C}(44)$ & $43(2)$ & $45(2)$ & $39(2)$ & $3(1)$ & $-6(1)$ & $-16(1)$ \\
\hline $\mathrm{C}(45)$ & $29(1)$ & $45(2)$ & $38(1)$ & $0(1)$ & $-3(1)$ & $-11(1)$ \\
\hline $\mathrm{C}(46)$ & $87(3)$ & $37(2)$ & $48(2)$ & $-2(1)$ & $-9(2)$ & $-12(2)$ \\
\hline $\mathrm{C}(47)$ & 21(1) & $27(1)$ & $28(1)$ & $-2(1)$ & 2(1) & $0(1)$ \\
\hline $\mathrm{C}(48)$ & 21(1) & $42(1)$ & $31(1)$ & 1(1) & 2(1) & $2(1)$ \\
\hline C(49) & $27(1)$ & $55(2)$ & $35(1)$ & $-3(1)$ & $7(1)$ & $5(1)$ \\
\hline $\mathrm{C}(50)$ & $35(1)$ & $61(2)$ & $29(1)$ & $-7(1)$ & $8(1)$ & 1(1) \\
\hline $\mathrm{C}(51)$ & $30(1)$ & $64(2)$ & $31(1)$ & $-2(1)$ & $0(1)$ & 1(1) \\
\hline $\mathrm{C}(52)$ & $24(1)$ & $46(2)$ & $30(1)$ & $-2(1)$ & 1(1) & $4(1)$ \\
\hline $\mathrm{C}(53)$ & $20(1)$ & $27(1)$ & $24(1)$ & $-1(1)$ & $-1(1)$ & $0(1)$ \\
\hline $\mathrm{C}(54)$ & $20(1)$ & $31(1)$ & $36(1)$ & $4(1)$ & $5(1)$ & $0(1)$ \\
\hline $\mathrm{C}(55)$ & $26(1)$ & $31(1)$ & 41(1) & $6(1)$ & $5(1)$ & $-2(1)$ \\
\hline $\mathrm{C}(56)$ & $29(1)$ & $26(1)$ & 41(1) & $1(1)$ & $0(1)$ & 2(1) \\
\hline $\mathrm{C}(57)$ & $20(1)$ & $29(1)$ & $42(1)$ & $-3(1)$ & $5(1)$ & $2(1)$ \\
\hline $\mathrm{C}(58)$ & $19(1)$ & $28(1)$ & $31(1)$ & $0(1)$ & $3(1)$ & $-3(1)$ \\
\hline $\mathrm{C}(59)$ & $24(1)$ & $27(1)$ & $29(1)$ & $2(1)$ & $5(1)$ & $-3(1)$ \\
\hline $\mathrm{C}(60)$ & $24(1)$ & $32(1)$ & $38(1)$ & $6(1)$ & 1(1) & $-2(1)$ \\
\hline C(61) & $37(1)$ & $34(1)$ & $46(2)$ & $11(1)$ & $4(1)$ & 2(1) \\
\hline
\end{tabular}




\begin{tabular}{|c|c|c|c|c|c|c|}
\hline$C(62)$ & $44(2)$ & $30(1)$ & $48(2)$ & $9(1)$ & $9(1)$ & $-7(1)$ \\
\hline $\mathrm{C}(63)$ & $35(1)$ & $38(1)$ & $48(2)$ & $3(1)$ & $5(1)$ & $-14(1)$ \\
\hline $\mathrm{C}(64)$ & $25(1)$ & $36(1)$ & $37(1)$ & $3(1)$ & $2(1)$ & $-5(1)$ \\
\hline$C(65)$ & $19(1)$ & $27(1)$ & $32(1)$ & 1(1) & $6(1)$ & $-5(1)$ \\
\hline $\mathrm{C}(66)$ & $20(1)$ & $43(1)$ & $33(1)$ & $-1(1)$ & $5(1)$ & $-2(1)$ \\
\hline$C(67)$ & $27(1)$ & $51(2)$ & $30(1)$ & $-2(1)$ & $6(1)$ & $-5(1)$ \\
\hline $\mathrm{C}(68)$ & $34(1)$ & $36(1)$ & $35(1)$ & $-6(1)$ & $14(1)$ & $-5(1)$ \\
\hline $\mathrm{C}(69)$ & $29(1)$ & $26(1)$ & $43(1)$ & $3(1)$ & 11(1) & $0(1)$ \\
\hline$C(70)$ & $25(1)$ & $26(1)$ & $33(1)$ & $5(1)$ & $5(1)$ & $0(1)$ \\
\hline $\mathrm{C}(71)$ & $23(1)$ & $25(1)$ & $27(1)$ & $0(1)$ & $4(1)$ & 1(1) \\
\hline$C(72)$ & $23(1)$ & $27(1)$ & $33(1)$ & $-2(1)$ & $2(1)$ & $3(1)$ \\
\hline$C(73)$ & $28(1)$ & $37(1)$ & $44(2)$ & $-2(1)$ & $3(1)$ & $8(1)$ \\
\hline$C(74)$ & $36(1)$ & $34(1)$ & $46(2)$ & $-10(1)$ & $6(1)$ & $8(1)$ \\
\hline$C(75)$ & $35(1)$ & $31(1)$ & $41(1)$ & $-7(1)$ & $2(1)$ & $-2(1)$ \\
\hline$C(76)$ & $23(1)$ & $27(1)$ & $34(1)$ & $-2(1)$ & $2(1)$ & $0(1)$ \\
\hline$C(77)$ & $18(1)$ & $29(1)$ & $31(1)$ & $4(1)$ & $3(1)$ & $-2(1)$ \\
\hline$C(78)$ & $30(1)$ & $37(1)$ & $42(2)$ & $-4(1)$ & $-1(1)$ & $6(1)$ \\
\hline$C(79)$ & $38(2)$ & $49(2)$ & $42(2)$ & $-10(1)$ & $-7(1)$ & $3(1)$ \\
\hline $\mathrm{C}(80)$ & $24(1)$ & $50(2)$ & $41(2)$ & $7(1)$ & $-5(1)$ & $-3(1)$ \\
\hline $\mathrm{C}(81)$ & $24(1)$ & $34(1)$ & $46(2)$ & $9(1)$ & $0(1)$ & $2(1)$ \\
\hline $\mathrm{C}(82)$ & $22(1)$ & $28(1)$ & $42(1)$ & $5(1)$ & $2(1)$ & $0(1)$ \\
\hline $\mathrm{C}(83)$ & $19(1)$ & $29(1)$ & $29(1)$ & $4(1)$ & $2(1)$ & $-2(1)$ \\
\hline $\mathrm{C}(84)$ & $31(1)$ & $28(1)$ & $48(2)$ & $6(1)$ & $13(1)$ & $2(1)$ \\
\hline$C(85)$ & $29(1)$ & $40(2)$ & $58(2)$ & $8(1)$ & $18(1)$ & $3(1)$ \\
\hline$C(86)$ & $29(1)$ & $36(1)$ & $53(2)$ & $8(1)$ & 11(1) & $-8(1)$ \\
\hline $\mathrm{C}(87)$ & $33(1)$ & $28(1)$ & $43(1)$ & $3(1)$ & $6(1)$ & $-4(1)$ \\
\hline $\mathrm{C}(88)$ & $27(1)$ & $29(1)$ & $34(1)$ & $2(1)$ & $6(1)$ & 1(1) \\
\hline C(89) & $22(1)$ & $27(1)$ & $30(1)$ & $3(1)$ & $4(1)$ & $4(1)$ \\
\hline $\mathrm{C}(90)$ & $26(1)$ & $38(1)$ & $34(1)$ & $2(1)$ & $4(1)$ & $-1(1)$ \\
\hline $\mathrm{C}(91)$ & $24(1)$ & $48(2)$ & $38(1)$ & $5(1)$ & $9(1)$ & $5(1)$ \\
\hline $\mathrm{C}(92)$ & $36(1)$ & $40(1)$ & $33(1)$ & 2(1) & $9(1)$ & $11(1)$ \\
\hline $\mathrm{C}(93)$ & $37(1)$ & $31(1)$ & $31(1)$ & $1(1)$ & $3(1)$ & $3(1)$ \\
\hline $\mathrm{C}(94)$ & $26(1)$ & $28(1)$ & $32(1)$ & $4(1)$ & $4(1)$ & $2(1)$ \\
\hline $\mathrm{C}(95)$ & $20(1)$ & $24(1)$ & $34(1)$ & $1(1)$ & $2(1)$ & $-2(1)$ \\
\hline $\mathrm{C}(96)$ & $20(1)$ & $30(1)$ & $38(1)$ & $0(1)$ & 2(1) & $2(1)$ \\
\hline $\mathrm{C}(97)$ & $19(1)$ & $34(1)$ & $45(1)$ & 2(1) & $1(1)$ & $-5(1)$ \\
\hline $\mathrm{C}(98)$ & $28(1)$ & $26(1)$ & $56(2)$ & $1(1)$ & $-1(1)$ & $-4(1)$ \\
\hline C(99) & $27(1)$ & $25(1)$ & $59(2)$ & $-2(1)$ & 1(1) & 1(1) \\
\hline $\mathrm{C}(100)$ & $20(1)$ & $27(1)$ & $44(1)$ & 1(1) & $3(1)$ & 1(1) \\
\hline $\mathrm{C}(101)$ & $20(1)$ & $25(1)$ & $31(1)$ & $2(1)$ & $3(1)$ & 1(1) \\
\hline $\mathrm{C}(102)$ & $21(1)$ & $38(1)$ & $32(1)$ & $3(1)$ & $2(1)$ & 1(1) \\
\hline $\mathrm{C}(103)$ & $20(1)$ & $49(2)$ & $36(1)$ & 2(1) & 1(1) & $-2(1)$ \\
\hline $\mathrm{C}(104)$ & $20(1)$ & $42(1)$ & $43(1)$ & $5(1)$ & $8(1)$ & $1(1)$ \\
\hline $\mathrm{C}(105)$ & $28(1)$ & $37(1)$ & $32(1)$ & 1(1) & $6(1)$ & $2(1)$ \\
\hline $\mathrm{C}(106)$ & $22(1)$ & $31(1)$ & $35(1)$ & $-1(1)$ & 1(1) & 1(1) \\
\hline $\mathrm{C}(107)$ & $15(1)$ & $29(1)$ & $31(1)$ & $3(1)$ & $4(1)$ & $2(1)$ \\
\hline $\mathrm{C}(108)$ & $18(1)$ & $28(1)$ & $36(1)$ & $3(1)$ & $3(1)$ & $0(1)$ \\
\hline $\mathrm{C}(109)$ & $22(1)$ & $38(1)$ & $34(1)$ & $9(1)$ & $4(1)$ & $2(1)$ \\
\hline $\mathrm{C}(110)$ & $20(1)$ & $44(1)$ & $31(1)$ & 1(1) & $3(1)$ & $5(1)$ \\
\hline $\mathrm{C}(111)$ & $21(1)$ & $34(1)$ & $37(1)$ & $-5(1)$ & $0(1)$ & $5(1)$ \\
\hline $\mathrm{C}(112)$ & $20(1)$ & $28(1)$ & $35(1)$ & 2(1) & $2(1)$ & $3(1)$ \\
\hline $\mathrm{C} 11$ & $47(2)$ & $68(2)$ & $64(2)$ & $-21(2)$ & $10(2)$ & $-4(2)$ \\
\hline $\mathrm{C} 21$ & $51(2)$ & $78(3)$ & $59(2)$ & $-5(2)$ & $5(2)$ & $7(2)$ \\
\hline C31 & $56(2)$ & $65(2)$ & $56(2)$ & $-3(2)$ & $6(2)$ & $1(2)$ \\
\hline $\mathrm{C} 41$ & $49(2)$ & $55(2)$ & $64(2)$ & $-15(2)$ & $5(2)$ & $-5(2)$ \\
\hline
\end{tabular}




\begin{tabular}{lllllll} 
C51 & $49(2)$ & $51(2)$ & $87(3)$ & $-8(2)$ & $5(2)$ & $-1(2)$ \\
C61 & $60(2)$ & $50(2)$ & $85(3)$ & $-16(2)$ & $7(2)$ & $-7(2)$ \\
C12 & $87(3)$ & $68(2)$ & $59(2)$ & $-12(2)$ & $17(2)$ & $-28(2)$ \\
C22 & $50(2)$ & $70(3)$ & $91(3)$ & $13(2)$ & $1(2)$ & $-10(2)$ \\
C32 & $58(2)$ & $64(2)$ & $98(3)$ & $-17(2)$ & $20(2)$ & $1(2)$ \\
C42 & $69(3)$ & $75(3)$ & $73(3)$ & $-13(2)$ & $11(2)$ & $-1(2)$ \\
C52 & $69(2)$ & $57(2)$ & $75(3)$ & $8(2)$ & $9(2)$ & $7(2)$ \\
C62 & $75(3)$ & $49(2)$ & $91(3)$ & $-12(2)$ & $15(2)$ & $9(2)$ \\
C13 & $42(2)$ & $53(2)$ & $196(7)$ & $39(3)$ & $24(3)$ & $-2(2)$ \\
C23 & $52(2)$ & $61(2)$ & $101(3)$ & $17(2)$ & $-6(2)$ & $-19(2)$ \\
C33 & $64(2)$ & $43(2)$ & $73(2)$ & $-4(2)$ & $13(2)$ & $-4(2)$ \\
C43 & $83(3)$ & $60(2)$ & $72(3)$ & $2(2)$ & $20(2)$ & $-20(2)$ \\
C53 & $166(6)$ & $80(3)$ & $89(3)$ & $-27(3)$ & $73(4)$ & $-54(4)$ \\
C63 & $119(5)$ & $49(3)$ & $188(7)$ & $-14(4)$ & $100(5)$ & $-11(3)$ \\
\hline
\end{tabular}

\subsection{Crystal Structure Determination of 6}

There are two molecules in the asymmetric unit. The hydrogen atoms at iridium were found in the difference Fourier map and refined independently.

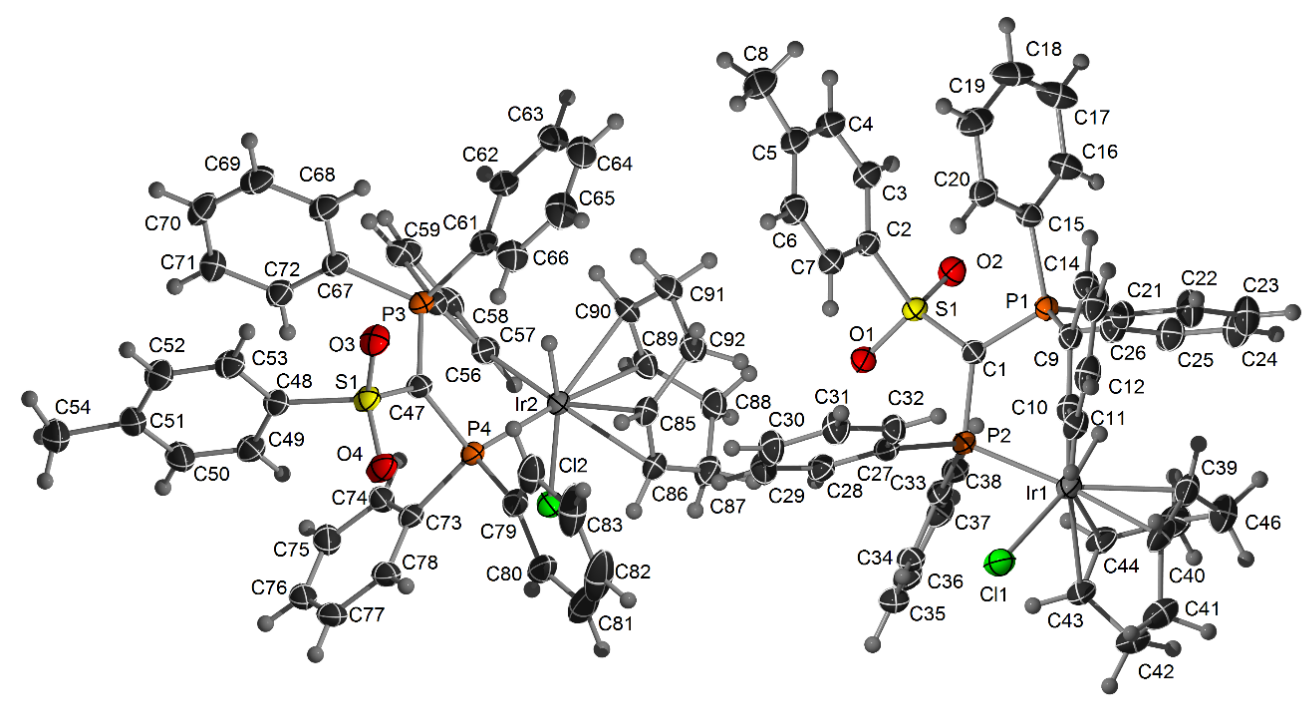

Figure S28. ORTEP Plot of 6. Ellipsoids are drawn at the 50\% probability level.

Table S23. Atomic coordinates ( $\left.\mathrm{x} 10^{4}\right)$ and equivalent isotropic displacement parameters $\left(\AA^{2} \times 10^{3}\right)$ for $6 . U(e q)$ is defined as one third of the trace of the orthogonalized $U i j$ tensor.

\begin{tabular}{lllll}
\hline & \multicolumn{1}{r}{$\mathrm{x}$} & $\mathrm{y}$ & $\mathrm{z}$ & $\mathrm{U}(\mathrm{eq})$ \\
\hline $\mathrm{Ir}(1)$ & $9663(1)$ & $3028(1)$ & $3962(1)$ & $26(1)$ \\
$\mathrm{Ir}(2)$ & $3185(1)$ & $6848(1)$ & $2138(1)$ & $23(1)$ \\
$\mathrm{Cl}(1)$ & $10564(1)$ & $4010(1)$ & $3039(1)$ & $35(1)$ \\
$\mathrm{Cl}(2)$ & $3146(1)$ & $8366(1)$ & $2582(1)$ & $31(1)$ \\
$\mathrm{S}(1)$ & $5744(1)$ & $2859(1)$ & $3872(1)$ & $24(1)$ \\
$\mathrm{S}(2)$ & $3450(1)$ & $7461(1)$ & $-97(1)$ & $27(1)$ \\
$\mathrm{P}(1)$ & $7657(1)$ & $1436(1)$ & $4076(1)$ & $24(1)$
\end{tabular}




\begin{tabular}{|c|c|c|c|c|}
\hline $\mathrm{P}(2)$ & $8006(1)$ & $3575(1)$ & $3657(1)$ & $23(1)$ \\
\hline $\mathrm{P}(3)$ & $1936(1)$ & $6523(1)$ & $947(1)$ & $24(1)$ \\
\hline $\mathrm{P}(4)$ & $3640(1)$ & $7726(1)$ & 1188(1) & $24(1)$ \\
\hline $\mathrm{O}(1)$ & $5544(2)$ & $3897(2)$ & $3702(1)$ & $28(1)$ \\
\hline $\mathrm{O}(2)$ & $5159(2)$ & $2426(2)$ & 4446(1) & $31(1)$ \\
\hline $\mathrm{O}(3)$ & $3500(2)$ & $6556(2)$ & $-340(1)$ & $33(1)$ \\
\hline $\mathrm{O}(4)$ & $4429(2)$ & $7959(2)$ & $-201(1)$ & $35(1)$ \\
\hline $\mathrm{C}(1)$ & 7124(3) & 2618(3) & $3853(2)$ & $25(1)$ \\
\hline$C(2)$ & 5301(3) & $2303(3)$ & $3318(2)$ & $26(1)$ \\
\hline$C(3)$ & 4449(3) & 1703(3) & $3499(2)$ & $33(1)$ \\
\hline $\mathrm{C}(4)$ & 4107(4) & $1273(3)$ & $3063(2)$ & $37(1)$ \\
\hline$C(5)$ & 4587(4) & 1434(3) & $2460(2)$ & $39(1)$ \\
\hline$C(6)$ & $5409(4)$ & 2071(3) & $2287(2)$ & $36(1)$ \\
\hline$C(7)$ & $5770(3)$ & $2505(3)$ & $2715(2)$ & $31(1)$ \\
\hline $\mathrm{C}(8)$ & $4259(5)$ & $897(4)$ & $2006(3)$ & $52(1)$ \\
\hline$C(9)$ & 8992(3) & 1153(3) & $3637(2)$ & $26(1)$ \\
\hline$C(10)$ & $9759(3)$ & 1847(3) & $3502(2)$ & $26(1)$ \\
\hline$C(11)$ & $10694(3)$ & 1644(3) & $3073(2)$ & $30(1)$ \\
\hline$C(12)$ & $10880(3)$ & $770(3)$ & 2831(2) & $34(1)$ \\
\hline$C(13)$ & 10163(4) & $58(3)$ & $3017(2)$ & $35(1)$ \\
\hline$C(14)$ & $9209(3)$ & $251(3)$ & $3413(2)$ & $30(1)$ \\
\hline$C(15)$ & $6829(3)$ & $534(3)$ & $3942(2)$ & $28(1)$ \\
\hline$C(16)$ & $6287(4)$ & $-73(3)$ & $4421(2)$ & $36(1)$ \\
\hline$C(17)$ & $5743(4)$ & $-822(4)$ & 4307(3) & $48(1)$ \\
\hline$C(18)$ & $5748(4)$ & $-953(4)$ & $3721(3)$ & $50(1)$ \\
\hline$C(19)$ & $6270(4)$ & $-339(3)$ & $3239(2)$ & $42(1)$ \\
\hline$C(20)$ & $6815(3)$ & $408(3)$ & $3346(2)$ & $32(1)$ \\
\hline $\mathrm{C}(21)$ & 7731(3) & 1191(3) & $4874(2)$ & $28(1)$ \\
\hline$C(22)$ & $8488(4)$ & $479(3)$ & $5088(2)$ & $38(1)$ \\
\hline$C(23)$ & $8548(4)$ & $326(4)$ & $5698(2)$ & $42(1)$ \\
\hline$C(24)$ & 7854(4) & $865(4)$ & 6091(2) & $40(1)$ \\
\hline$C(25)$ & 7098(4) & 1557(3) & $5886(2)$ & $35(1)$ \\
\hline$C(26)$ & 7034(3) & 1731(3) & $5275(2)$ & $31(1)$ \\
\hline$C(27)$ & $8026(3)$ & $3979(3)$ & $2846(2)$ & $25(1)$ \\
\hline $\mathrm{C}(28)$ & 7415(3) & $4795(3)$ & $2626(2)$ & $29(1)$ \\
\hline$C(29)$ & 7423(4) & $5033(3)$ & $2007(2)$ & $37(1)$ \\
\hline$C(30)$ & 8019(4) & $4442(3)$ & 1611(2) & $38(1)$ \\
\hline$C(31)$ & $8616(4)$ & $3613(3)$ & $1829(2)$ & $35(1)$ \\
\hline$C(32)$ & $8631(3)$ & $3384(3)$ & $2442(2)$ & $29(1)$ \\
\hline C(33) & $7430(3)$ & $4659(3)$ & $4011(2)$ & $27(1)$ \\
\hline$C(34)$ & 7864(3) & $5546(3)$ & $3764(2)$ & $29(1)$ \\
\hline$C(35)$ & $7478(3)$ & $6382(3)$ & 4031(2) & $32(1)$ \\
\hline$C(36)$ & $6700(3)$ & $6336(3)$ & $4547(2)$ & $33(1)$ \\
\hline$C(37)$ & 6311(3) & $5446(3)$ & $4814(2)$ & $29(1)$ \\
\hline C(38) & $6665(3)$ & $4612(3)$ & $4539(2)$ & $28(1)$ \\
\hline C(39) & $10700(4)$ & $2125(4)$ & $4594(2)$ & $38(1)$ \\
\hline$C(40)$ & $11386(3)$ & $2580(3)$ & $4114(2)$ & $35(1)$ \\
\hline$C(41)$ & 11971(4) & $3456(4)$ & $4134(2)$ & $45(1)$ \\
\hline$C(42)$ & $11266(4)$ & $4271(3)$ & $4416(2)$ & $40(1)$ \\
\hline C(43) & $10066(4)$ & $4326(3)$ & $4372(2)$ & $33(1)$ \\
\hline$C(44)$ & $9312(4)$ & $3834(3)$ & $4798(2)$ & $33(1)$ \\
\hline$C(45)$ & $9540(4)$ & $3149(4)$ & $5345(2)$ & $41(1)$ \\
\hline C(46) & $10489(4)$ & 2412(4) & $5225(2)$ & $45(1)$ \\
\hline $\mathrm{C}(47)$ & $3008(3)$ & $7260(3)$ & $673(2)$ & $25(1)$ \\
\hline C(48) & 2555(3) & $8291(3)$ & $-488(2)$ & $28(1)$ \\
\hline
\end{tabular}




\begin{tabular}{lllll}
$\mathrm{C}(49)$ & $2223(4)$ & $9210(3)$ & $-319(2)$ & $34(1)$ \\
$\mathrm{C}(50)$ & $1657(4)$ & $9876(3)$ & $-679(2)$ & $40(1)$ \\
$\mathrm{C}(51)$ & $1421(4)$ & $9666(3)$ & $-1218(2)$ & $40(1)$ \\
$\mathrm{C}(52)$ & $1735(4)$ & $8748(3)$ & $-1368(2)$ & $38(1)$ \\
$\mathrm{C}(53)$ & $2293(3)$ & $8046(3)$ & $-1010(2)$ & $33(1)$ \\
$\mathrm{C}(54)$ & $877(5)$ & $10439(4)$ & $-1631(2)$ & $51(1)$ \\
$\mathrm{C}(55)$ & $1083(3)$ & $6854(3)$ & $1636(2)$ & $24(1)$ \\
$\mathrm{C}(56)$ & $1553(3)$ & $7133(3)$ & $2092(2)$ & $25(1)$ \\
$\mathrm{C}(57)$ & $820(3)$ & $7454(3)$ & $2582(2)$ & $28(1)$ \\
$\mathrm{C}(58)$ & $-290(4)$ & $7415(3)$ & $2645(2)$ & $33(1)$ \\
$\mathrm{C}(59)$ & $-721(3)$ & $7054(3)$ & $2213(2)$ & $34(1)$ \\
$\mathrm{C}(60)$ & $-39(3)$ & $6798(3)$ & $1704(2)$ & $31(1)$ \\
$\mathrm{C}(61)$ & $2317(3)$ & $5225(3)$ & $1074(2)$ & $29(1)$ \\
$\mathrm{C}(62)$ & $1597(4)$ & $4605(3)$ & $1443(2)$ & $32(1)$ \\
$\mathrm{C}(63)$ & $1896(4)$ & $3612(3)$ & $1550(2)$ & $38(1)$ \\
$\mathrm{C}(64)$ & $2915(4)$ & $3241(3)$ & $1289(2)$ & $44(1)$ \\
$\mathrm{C}(65)$ & $3633(4)$ & $3851(3)$ & $919(2)$ & $44(1)$ \\
$\mathrm{C}(66)$ & $3336(4)$ & $4842(3)$ & $808(2)$ & $36(1)$ \\
$\mathrm{C}(67)$ & $1038(3)$ & $6723(3)$ & $396(2)$ & $28(1)$ \\
$\mathrm{C}(68)$ & $924(3)$ & $6002(3)$ & $65(2)$ & $34(1)$ \\
$\mathrm{C}(69)$ & $221(4)$ & $6198(4)$ & $-353(2)$ & $41(1)$ \\
$\mathrm{C}(70)$ & $-337(4)$ & $7096(4)$ & $-445(2)$ & $39(1)$ \\
$\mathrm{C}(71)$ & $-217(4)$ & $7825(3)$ & $-119(2)$ & $36(1)$ \\
$\mathrm{C}(72)$ & $470(3)$ & $7633(3)$ & $303(2)$ & $31(1)$ \\
$\mathrm{C}(73)$ & $3273(3)$ & $9052(3)$ & $1076(2)$ & $29(1)$ \\
$\mathrm{C}(74)$ & $2238(4)$ & $9398(3)$ & $1327(2)$ & $31(1)$ \\
$\mathrm{C}(75)$ & $1871(4)$ & $10375(3)$ & $1178(2)$ & $36(1)$ \\
$\mathrm{C}(76)$ & $2546(4)$ & $10995(3)$ & $784(2)$ & $37(1)$ \\
$\mathrm{C}(77)$ & $3568(4)$ & $10675(3)$ & $543(2)$ & $38(1)$ \\
$\mathrm{C}(78)$ & $3947(4)$ & $9692(3)$ & $682(2)$ & $34(1)$ \\
$\mathrm{C}(79)$ & $5114(3)$ & $7603(3)$ & $973(2)$ & $34(1)$ \\
$\mathrm{C}(80)$ & $5710(4)$ & $8180(4)$ & $1220(2)$ & $44(1)$ \\
$\mathrm{C}(81)$ & $6834(5)$ & $8015(6)$ & $1140(3)$ & $66(2)$ \\
$\mathrm{C}(82)$ & $7356(4)$ & $7299(6)$ & $830(3)$ & $73(2)$ \\
$\mathrm{C}(83)$ & $6786(4)$ & $6714(5)$ & $573(2)$ & $61(2)$ \\
$\mathrm{C}(84)$ & $5660(4)$ & $6869(4)$ & $653(2)$ & $42(1)$ \\
$\mathrm{C}(85)$ & $4797(3)$ & $5879(3)$ & $2112(2)$ & $29(1)$ \\
$\mathrm{C}(86)$ & $4860(3)$ & $6614(3)$ & $2435(2)$ & $29(1)$ \\
$\mathrm{C}(87)$ & $4725(4)$ & $6520(3)$ & $3111(2)$ & $34(1)$ \\
$\mathrm{C}(88)$ & $3758(4)$ & $5969(3)$ & $3445(2)$ & $37(1)$ \\
$\mathrm{C}(89)$ & $2795(4)$ & $6099(3)$ & $3111(2)$ & $31(1)$ \\
$\mathrm{C}(90)$ & $2661(3)$ & $5456(3)$ & $2730(2)$ & $30(1)$ \\
$\mathrm{C}(91)$ & $3450(4)$ & $4604(3)$ & $2561(2)$ & $34(1)$ \\
$\mathrm{C}(92)$ & $4630(4)$ & $4837(3)$ & $2391(2)$ & $33(1)$ \\
\hline & & & & \\
\hline
\end{tabular}

Table S24. Anisotropic displacement parameters $\left(\AA^{2} \times 10^{3}\right)$ for $\mathbf{6}$. The anisotropic displacement factor exponent takes the form: $-2 \pi^{2}\left[h^{2} a^{* 2} U^{11}+\ldots+2 h k a^{*} b^{*} U^{12}\right]$

\begin{tabular}{lllllll}
\hline & $\mathrm{U}^{11}$ & $\mathrm{U}^{22}$ & $\mathrm{U}^{33}$ & $\mathrm{U}^{23}$ & $\mathrm{U}^{13}$ & \multicolumn{1}{c}{$\mathrm{U}^{12}$} \\
\hline $\mathrm{Ir}(1)$ & $26(1)$ & $29(1)$ & $24(1)$ & $-6(1)$ & $-8(1)$ & $-4(1)$ \\
$\mathrm{Ir}(2)$ & $28(1)$ & $22(1)$ & $21(1)$ & $-3(1)$ & $-8(1)$ & $-4(1)$ \\
$\mathrm{Cl}(1)$ & $36(1)$ & $40(1)$ & $31(1)$ & $-4(1)$ & $-6(1)$ & $-14(1)$ \\
$\mathrm{Cl}(2)$ & $45(1)$ & $26(1)$ & $26(1)$ & $-5(1)$ & $-14(1)$ & $-5(1)$
\end{tabular}




\begin{tabular}{|c|c|c|c|c|c|c|}
\hline$S(1)$ & $26(1)$ & $25(1)$ & $23(1)$ & $-5(1)$ & $-7(1)$ & $-2(1)$ \\
\hline$S(2)$ & $29(1)$ & $32(1)$ & $22(1)$ & $-5(1)$ & $-6(1)$ & $-6(1)$ \\
\hline $\mathrm{P}(1)$ & $28(1)$ & $22(1)$ & $21(1)$ & $-2(1)$ & $-7(1)$ & $-2(1)$ \\
\hline $\mathrm{P}(2)$ & $27(1)$ & $22(1)$ & $21(1)$ & $-4(1)$ & $-7(1)$ & $-4(1)$ \\
\hline $\mathrm{P}(3)$ & $26(1)$ & 23(1) & $24(1)$ & $-5(1)$ & $-7(1)$ & $-5(1)$ \\
\hline $\mathrm{P}(4)$ & $28(1)$ & $25(1)$ & $22(1)$ & $-2(1)$ & $-9(1)$ & $-8(1)$ \\
\hline $\mathrm{O}(1)$ & $32(1)$ & $24(1)$ & $32(1)$ & $-7(1)$ & $-12(1)$ & $1(1)$ \\
\hline $\mathrm{O}(2)$ & $29(1)$ & $38(2)$ & $26(1)$ & $-3(1)$ & $-4(1)$ & $-5(1)$ \\
\hline $\mathrm{O}(3)$ & $37(2)$ & $35(2)$ & $29(1)$ & $-12(1)$ & $-7(1)$ & 1(1) \\
\hline $\mathrm{O}(4)$ & $33(2)$ & $48(2)$ & $26(1)$ & $-2(1)$ & $-6(1)$ & $-13(1)$ \\
\hline$C(1)$ & $29(2)$ & $23(2)$ & $23(2)$ & $-4(1)$ & $-7(1)$ & $-3(1)$ \\
\hline$C(2)$ & $27(2)$ & $25(2)$ & $28(2)$ & $-6(1)$ & $-10(2)$ & $0(1)$ \\
\hline$C(3)$ & $29(2)$ & $35(2)$ & $37(2)$ & $-5(2)$ & $-10(2)$ & $-5(2)$ \\
\hline$C(4)$ & $36(2)$ & $33(2)$ & $46(3)$ & $-4(2)$ & $-16(2)$ & $-9(2)$ \\
\hline$C(5)$ & $48(3)$ & $30(2)$ & $48(3)$ & $-8(2)$ & $-26(2)$ & $-5(2)$ \\
\hline$C(6)$ & $47(2)$ & $34(2)$ & $30(2)$ & $-7(2)$ & $-14(2)$ & $-2(2)$ \\
\hline$C(7)$ & $35(2)$ & $29(2)$ & $30(2)$ & $-3(2)$ & $-11(2)$ & $-5(2)$ \\
\hline $\mathrm{C}(8)$ & $70(3)$ & $44(3)$ & $54(3)$ & $-13(2)$ & $-33(3)$ & $-10(2)$ \\
\hline $\mathrm{C}(9)$ & $30(2)$ & $27(2)$ & $22(2)$ & $-4(1)$ & $-9(1)$ & $-1(1)$ \\
\hline $\mathrm{C}(10)$ & $31(2)$ & $28(2)$ & $21(2)$ & $-10(1)$ & $-10(1)$ & $3(1)$ \\
\hline $\mathrm{C}(11)$ & $29(2)$ & $37(2)$ & $25(2)$ & $-5(2)$ & $-7(2)$ & $-2(2)$ \\
\hline$C(12)$ & $35(2)$ & $45(2)$ & $24(2)$ & $-12(2)$ & $-9(2)$ & $8(2)$ \\
\hline$C(13)$ & $39(2)$ & $41(2)$ & $29(2)$ & $-12(2)$ & $-14(2)$ & $6(2)$ \\
\hline$C(14)$ & $34(2)$ & $30(2)$ & $29(2)$ & $-6(2)$ & $-11(2)$ & $1(2)$ \\
\hline$C(15)$ & $30(2)$ & $21(2)$ & $34(2)$ & $-6(2)$ & $-8(2)$ & $-3(1)$ \\
\hline$C(16)$ & $39(2)$ & $29(2)$ & $38(2)$ & $-3(2)$ & $-2(2)$ & $-9(2)$ \\
\hline$C(17)$ & $50(3)$ & $33(2)$ & $60(3)$ & $-5(2)$ & $1(2)$ & $-17(2)$ \\
\hline $\mathrm{C}(18)$ & $48(3)$ & $34(2)$ & $72(4)$ & $-18(2)$ & $-6(2)$ & $-16(2)$ \\
\hline $\mathrm{C}(19)$ & $43(2)$ & $40(2)$ & $49(3)$ & $-19(2)$ & $-11(2)$ & $-6(2)$ \\
\hline $\mathrm{C}(20)$ & $32(2)$ & $30(2)$ & $37(2)$ & $-8(2)$ & $-10(2)$ & $-2(2)$ \\
\hline $\mathrm{C}(21)$ & $32(2)$ & $28(2)$ & $25(2)$ & $-1(1)$ & $-7(2)$ & $-4(2)$ \\
\hline $\mathrm{C}(22)$ & $41(2)$ & $40(2)$ & $30(2)$ & $-5(2)$ & $-7(2)$ & $8(2)$ \\
\hline $\mathrm{C}(23)$ & $48(3)$ & $44(3)$ & $34(2)$ & $-4(2)$ & $-16(2)$ & $11(2)$ \\
\hline $\mathrm{C}(24)$ & $45(2)$ & $50(3)$ & $24(2)$ & $-5(2)$ & $-9(2)$ & $1(2)$ \\
\hline $\mathrm{C}(25)$ & $39(2)$ & $40(2)$ & $28(2)$ & $-6(2)$ & $-7(2)$ & $-1(2)$ \\
\hline $\mathrm{C}(26)$ & $34(2)$ & $32(2)$ & $29(2)$ & $-6(2)$ & $-10(2)$ & $0(2)$ \\
\hline $\mathrm{C}(27)$ & $29(2)$ & $26(2)$ & $23(2)$ & $-2(1)$ & $-7(1)$ & $-9(1)$ \\
\hline $\mathrm{C}(28)$ & $32(2)$ & $31(2)$ & $27(2)$ & $-6(2)$ & $-7(2)$ & $-4(2)$ \\
\hline $\mathrm{C}(29)$ & $40(2)$ & $37(2)$ & $33(2)$ & $0(2)$ & $-12(2)$ & $1(2)$ \\
\hline $\mathrm{C}(30)$ & $49(3)$ & $43(2)$ & $24(2)$ & $-2(2)$ & $-13(2)$ & $-1(2)$ \\
\hline $\mathrm{C}(31)$ & $45(2)$ & $37(2)$ & $25(2)$ & $-8(2)$ & $-9(2)$ & $1(2)$ \\
\hline $\mathrm{C}(32)$ & $38(2)$ & $28(2)$ & $23(2)$ & $-3(1)$ & $-11(2)$ & $-1(2)$ \\
\hline $\mathrm{C}(33)$ & $31(2)$ & $27(2)$ & $26(2)$ & $-6(1)$ & $-11(2)$ & $-2(2)$ \\
\hline $\mathrm{C}(34)$ & $34(2)$ & $27(2)$ & $28(2)$ & $-6(2)$ & $-7(2)$ & $-7(2)$ \\
\hline $\mathrm{C}(35)$ & $36(2)$ & $28(2)$ & $35(2)$ & $-6(2)$ & $-9(2)$ & $-6(2)$ \\
\hline $\mathrm{C}(36)$ & $36(2)$ & $30(2)$ & $39(2)$ & $-15(2)$ & $-16(2)$ & $0(2)$ \\
\hline $\mathrm{C}(37)$ & $27(2)$ & $38(2)$ & $26(2)$ & $-13(2)$ & $-6(2)$ & $1(2)$ \\
\hline $\mathrm{C}(38)$ & $32(2)$ & $27(2)$ & $26(2)$ & $-6(2)$ & $-8(2)$ & $-6(2)$ \\
\hline C(39) & $38(2)$ & $43(2)$ & $38(2)$ & $-7(2)$ & $-22(2)$ & $4(2)$ \\
\hline $\mathrm{C}(40)$ & $23(2)$ & $48(2)$ & $39(2)$ & $-16(2)$ & $-13(2)$ & $5(2)$ \\
\hline $\mathrm{C}(41)$ & $32(2)$ & $55(3)$ & $55(3)$ & $-18(2)$ & $-14(2)$ & $-6(2)$ \\
\hline $\mathrm{C}(42)$ & $38(2)$ & $40(2)$ & $48(3)$ & $-8(2)$ & $-13(2)$ & $-14(2)$ \\
\hline $\mathrm{C}(43)$ & $38(2)$ & $31(2)$ & $36(2)$ & $-9(2)$ & $-15(2)$ & $-6(2)$ \\
\hline $\mathrm{C}(44)$ & $36(2)$ & $40(2)$ & $27(2)$ & $-12(2)$ & $-9(2)$ & $-8(2)$ \\
\hline $\mathrm{C}(45)$ & $52(3)$ & $47(3)$ & $28(2)$ & $-10(2)$ & $-11(2)$ & $-10(2)$ \\
\hline
\end{tabular}




\begin{tabular}{|c|c|c|c|c|c|c|}
\hline C(46) & $48(3)$ & $53(3)$ & $36(2)$ & $-5(2)$ & $-16(2)$ & $0(2)$ \\
\hline C(47) & $28(2)$ & $25(2)$ & $25(2)$ & $-4(1)$ & $-7(1)$ & $-7(1)$ \\
\hline C(48) & $36(2)$ & $30(2)$ & $21(2)$ & $-2(1)$ & $-8(2)$ & $-8(2)$ \\
\hline C(49) & $46(2)$ & $33(2)$ & $29(2)$ & $-6(2)$ & $-14(2)$ & $-9(2)$ \\
\hline$C(50)$ & $61(3)$ & $28(2)$ & $37(2)$ & $-4(2)$ & $-20(2)$ & $-6(2)$ \\
\hline $\mathrm{C}(51)$ & $52(3)$ & $34(2)$ & $35(2)$ & $5(2)$ & $-19(2)$ & $-12(2)$ \\
\hline$C(52)$ & $50(3)$ & $41(2)$ & $26(2)$ & $-4(2)$ & $-13(2)$ & $-9(2)$ \\
\hline C(53) & $39(2)$ & $37(2)$ & $26(2)$ & $-8(2)$ & $-7(2)$ & $-7(2)$ \\
\hline$C(54)$ & $70(3)$ & $40(3)$ & $47(3)$ & $4(2)$ & $-31(3)$ & $-9(2)$ \\
\hline C(55) & $27(2)$ & $20(2)$ & $26(2)$ & 1(1) & $-8(1)$ & $-2(1)$ \\
\hline$C(56)$ & $25(2)$ & $22(2)$ & $28(2)$ & $-2(1)$ & $-8(1)$ & $-1(1)$ \\
\hline C(57) & $33(2)$ & $26(2)$ & $24(2)$ & $-5(1)$ & $-4(2)$ & $-1(2)$ \\
\hline C(58) & $40(2)$ & $29(2)$ & $26(2)$ & $-1(2)$ & $1(2)$ & $0(2)$ \\
\hline C(59) & $26(2)$ & $35(2)$ & $39(2)$ & 1(2) & $-4(2)$ & $-4(2)$ \\
\hline$C(60)$ & $31(2)$ & $31(2)$ & $30(2)$ & $-1(2)$ & $-6(2)$ & $-6(2)$ \\
\hline $\mathrm{C}(61)$ & $37(2)$ & $26(2)$ & $28(2)$ & $-9(2)$ & $-12(2)$ & $-3(2)$ \\
\hline$C(62)$ & $38(2)$ & $28(2)$ & $35(2)$ & $-7(2)$ & $-13(2)$ & $-6(2)$ \\
\hline $\mathrm{C}(63)$ & $56(3)$ & $26(2)$ & $38(2)$ & $-4(2)$ & $-18(2)$ & $-10(2)$ \\
\hline$C(64)$ & $67(3)$ & $25(2)$ & $48(3)$ & $-12(2)$ & $-29(2)$ & $4(2)$ \\
\hline$C(65)$ & $54(3)$ & $36(2)$ & $45(3)$ & $-18(2)$ & $-15(2)$ & $11(2)$ \\
\hline$C(66)$ & $39(2)$ & $33(2)$ & $36(2)$ & $-9(2)$ & $-6(2)$ & $1(2)$ \\
\hline $\mathrm{C}(67)$ & $28(2)$ & $33(2)$ & $27(2)$ & $-6(2)$ & $-8(2)$ & $-7(2)$ \\
\hline$C(68)$ & $36(2)$ & $38(2)$ & $33(2)$ & $-10(2)$ & $-10(2)$ & $-8(2)$ \\
\hline C(69) & $45(2)$ & $46(2)$ & $37(2)$ & $-14(2)$ & $-13(2)$ & $-12(2)$ \\
\hline$C(70)$ & $36(2)$ & $57(3)$ & $30(2)$ & $-7(2)$ & $-15(2)$ & $-8(2)$ \\
\hline$C(71)$ & $38(2)$ & $39(2)$ & $32(2)$ & $-2(2)$ & $-13(2)$ & $-3(2)$ \\
\hline$C(72)$ & $31(2)$ & $36(2)$ & $27(2)$ & $-3(2)$ & $-8(2)$ & $-7(2)$ \\
\hline$C(73)$ & $37(2)$ & $30(2)$ & $24(2)$ & $-4(2)$ & $-14(2)$ & $-10(2)$ \\
\hline$C(74)$ & $42(2)$ & $25(2)$ & $30(2)$ & $0(2)$ & $-13(2)$ & $-6(2)$ \\
\hline$C(75)$ & $46(2)$ & $30(2)$ & $36(2)$ & $-5(2)$ & $-16(2)$ & $-1(2)$ \\
\hline$C(76)$ & $53(3)$ & $25(2)$ & $38(2)$ & $-2(2)$ & $-22(2)$ & $-8(2)$ \\
\hline$C(77)$ & $53(3)$ & $30(2)$ & $37(2)$ & $0(2)$ & $-17(2)$ & $-18(2)$ \\
\hline $\mathrm{C}(78)$ & $45(2)$ & $30(2)$ & $29(2)$ & $-1(2)$ & $-12(2)$ & $-14(2)$ \\
\hline$C(79)$ & $33(2)$ & $40(2)$ & $30(2)$ & $4(2)$ & $-13(2)$ & $-10(2)$ \\
\hline $\mathrm{C}(80)$ & $43(2)$ & $55(3)$ & $38(2)$ & $13(2)$ & $-18(2)$ & $-26(2)$ \\
\hline $\mathrm{C}(81)$ & $45(3)$ & $102(5)$ & $54(3)$ & $28(3)$ & $-26(3)$ & $-38(3)$ \\
\hline $\mathrm{C}(82)$ & $24(2)$ & $129(6)$ & $52(3)$ & $35(4)$ & $-9(2)$ & $-13(3)$ \\
\hline $\mathrm{C}(83)$ & $30(2)$ & $104(5)$ & $35(3)$ & $16(3)$ & $-1(2)$ & $10(3)$ \\
\hline$C(84)$ & $31(2)$ & $60(3)$ & $30(2)$ & $6(2)$ & $-5(2)$ & $0(2)$ \\
\hline$C(85)$ & $26(2)$ & $31(2)$ & $30(2)$ & $-4(2)$ & $-10(2)$ & $2(2)$ \\
\hline$C(86)$ & $25(2)$ & $33(2)$ & $32(2)$ & $-2(2)$ & $-12(2)$ & $-2(2)$ \\
\hline C(87) & $38(2)$ & $37(2)$ & $31(2)$ & $-8(2)$ & $-16(2)$ & $-1(2)$ \\
\hline $\mathrm{C}(88)$ & $48(3)$ & $37(2)$ & $26(2)$ & $-2(2)$ & $-14(2)$ & $-3(2)$ \\
\hline C(89) & $39(2)$ & $30(2)$ & $22(2)$ & $2(2)$ & $-6(2)$ & $-2(2)$ \\
\hline $\mathrm{C}(90)$ & $36(2)$ & $23(2)$ & $30(2)$ & $7(2)$ & $-8(2)$ & $-8(2)$ \\
\hline C(91) & $44(2)$ & $21(2)$ & $39(2)$ & $-1(2)$ & $-11(2)$ & $-4(2)$ \\
\hline $\mathrm{C}(92)$ & $41(2)$ & $28(2)$ & $32(2)$ & $-5(2)$ & $-11(2)$ & $1(2)$ \\
\hline
\end{tabular}

